\title{
Reynolds-number effects and anisotropy in transverse-jet mixing
}

\author{
By JERRY W. SHAN AND PAUL E. DIMOTAKIS \\ Graduate Aeronautical Laboratories, California Institute of Technology, Pasadena, CA 91125, USA
}

(Received 23 May 2005 and in revised form 8 January 2006)

Experiments are described which measured concentration fields in liquid-phase strong transverse jets over the Reynolds-number range $1.0 \times 10^{3} \leqslant R e_{j} \leqslant 20 \times 10^{3}$. Laserinduced-fluorescence measurements were made of the jet-fluid-concentration fields at a jet-to-freestream velocity ratio of $V_{r}=10$. The concentration-field data for far-field $\left(x / d_{j}=50\right)$ slices of the jet show that turbulent mixing in the transverse jet is Reynoldsnumber dependent over the range investigated, with a scalar-field PDF that evolves with Reynolds number. A growing peak in the PDF, indicating enhanced spatial homogenization of the jet-fluid concentration field, is found with increasing Reynolds number. Comparisons between transverse jets and jets discharging into quiescent reservoirs show that the transverse jet is an efficient mixer in that it entrains more fluid than the ordinary jet, yet is able to effectively mix and homogenize the additional entrained fluid. Analysis of the structure of the scalar field using distributions of scalar increments shows evidence for well-mixed plateaux separated by sharp cliffs in the jet-fluid concentration field, as previously shown in other flows. Furthermore, the scalar field is found to be anisotropic, even at small length scales. Evidence for local anisotropy is seen in the scalar power spectra, scalar microscales, and PDFs of scalar increments in different directions. The scalar-field anisotropy is shown to be correlated to the vortex-induced large-scale strain field of the transverse jet. These experiments add to the existing evidence that the large and small scales of high-Schmidt-number turbulent mixing flows can be linked, with attendant consequences for the universality of small scales of the scalar field for Reynolds numbers up to at least $R e=20 \times 10^{4}$.

\section{Introduction}

The turbulent jet discharging into a crossflow, or transverse jet, is a turbulent freeshear flow of both environmental and technological significance. A common-place occurrence of the transverse jet is the plume emitted from a smokestack on a windy day. Plumes generated by volcanoes, thunderstorms, or forest fires can also rise to heights in the atmosphere where significant crossflow exists. Other transverse jets arise in diverse situations including effluent discharge into rivers, steering jets for missiles and ships, VTOL/STOL aircraft aerodynamics, and blade-and-endwall cooling in gas turbines. The transverse jet has also been proposed as a means for fuel injection in high-speed air-breathing propulsion, i.e. SCRAMJETS (Gruber et al.1999). Buoyancy can be important to different degrees in the various applications of transverse jets; however, this study restricts its attention to momentum-driven jets in crossflow.

A large body of work has focused on the velocity fields and vortex dynamics of the turbulent jet in crossflow (Gordier 1959; Keffer \& Baines 1963; McMahon, Hester \& Palfery 1971; Chassaing et al. 1974; Fearn \& Weston 1974; Moussa, Trischka \& 
Eskinazl 1977; Andreoupoulos \& Rodi 1984; Karagozian 1986; Kelso \& Smits 1995; Kelso, Lim \& Perry 1996; Cortelezzi \& Karagozian 2001; extensive literature reviews are given by Margason 1993 and Morton \& Ibbetson 1996). In comparison, less attention has been paid to scalar transport and mixing, despite the fact that, as noted by Niederhaus, Champangne \& Jacobs (1997), 'the majority of applications require knowledge of the transport of either heat or mass.' A number of early studies focused on classical measures of jet mixing such as scalar trajectories, centreline concentration decay, and mean scalar fields (e.g. Patrick 1967; Kamotani \& Greber 1972). Broadwell \& Breidenthal (1984) made an important contribution by modelling the transverse jet as an axial vortex pair that arises as a global consequence of the transverse lift force imparted by the jet to the crossflow. The analysis of Broadwell \& Breidenthal provided analytical models for the rate at which mean concentration decays on the centreline. Broadwell \& Breidenthal also performed experiments measuring 'flame length' in liquid-phase transverse jets, and reported the flame length to be independent of Reynolds number above a circulation-based Reynolds number of $\Gamma / v \simeq 300$.

Smith \& Mungal (1998) reported on experiments on the mixing and structure of gas-phase transverse jets. They identified different regions of the transverse jet for which different scalings held: the vortex interaction region, the near field, and the far field. Based on decay rates of mean concentration on the jet centreline, they found the location of a branch point separating the near and far fields to be insensitive to Reynolds number over the range $8.4 \times 10^{3} \leqslant R e_{j} \leqslant 33 \times 10^{3}$, where Reynolds number is based on jet-exit velocity and nozzle diameter. Hasselbrink \& Mungal (2001) used similarity analysis to find scaling laws for the mean centreline concentration decay in the near and far fields. Reasonable agreement was found with the data of Smith \& Mungal (1998). Su \& Mungal (2004) have have reported on simultaneous measurements of velocity and scalar fields in transverse jets. Other studies of turbulent mixing in transverse jets with additional complexity have been reported, e.g. for jets with swirl (Niederhaus et al. 1997), sonic jets injected into a supersonic crossflow (VanLerberghe et al.2000), and fully modulated jets (Johari, Pacheco-Tougas \& Hermanson 1999).

Relatively little attention appears to have been paid to the evolution of mixing with Reynolds number in transverse jets. Smith \& Mungal (1998) found that the mean concentration decay rate on the jet centreline was approximately constant over a Reynolds-number range of $8.4 \times 10^{3} \leqslant R e_{j} \leqslant 33 \times 10^{3}$. However, no comparison was made of probability distribution functions (PDFs) of the scalar field for different Reynolds numbers. As discussed in $\S 3$, mean quantities such as the mean concentration decay rate are measures of entrainment, rather than measures of mixing. Moreover, experience with liquid-phase axisymmetric turbulent jets discharging into a quiescent reservoir shows that the details of scalar fields mixed by turbulence can depend on Reynolds number. For example, scalar-field distributions and root-meansquared (r.m.s.) scalar fluctuations vary with jet Reynolds number over a range of at least $3.0 \times 10^{3} \leqslant R e_{j} \leqslant 24 \times 10^{3}$ (Miller \& Dimotakis 1991; Catrakis \& Dimotakis 1996). This paper addresses the issue of Reynolds-number dependence of scalar mixing by examining the probability distribution of jet fluid in strong liquid-phase transverse jets at a fixed far-downstream location ( $(3)$. In addition, high-Schmidt-number mixing is compared between transverse jets and ordinary jets to investigate possible differences in mixing for fully developed (but finite-Reynolds-number) turbulent flows.

A detailed examination of the structure of the mixed-fluid concentration fields is also made with scalar increments (also known as scalar differences). The distribution of 


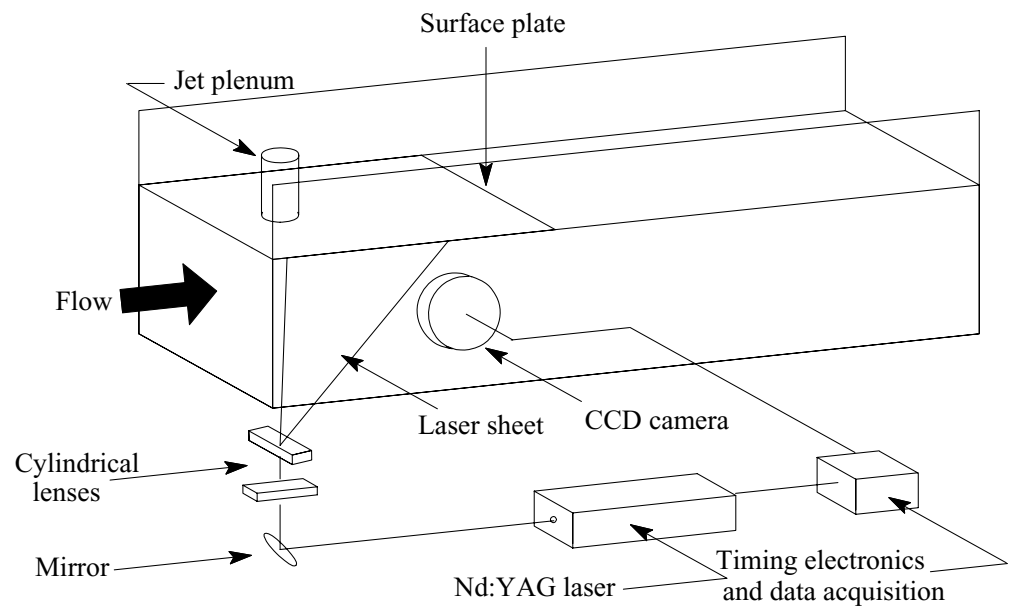

FIGURE 1. Experimental facility and imaging configuration for streamwise views of a transverse jet.

scalar increments, $f\left(\Delta_{r} C\right)$, defined as the probability distribution of the instantaneous concentration difference between two spatially separated points in the scalar field,

$$
f\left(\Delta_{r} C\right) \equiv f(C(\boldsymbol{x}+\boldsymbol{r}, t)-C(\boldsymbol{x}, t)),
$$

describes the probability of finding a concentration $C+\Delta C$ at a distance $r$ away from a point of concentration $C$. The scalar increment statistics, which are connected to intermittency and the structure of the scalar field, are examined in $\S 4$ to explore the internal structure of the jet-fluid concentration field.

The experiments reported here also examine other aspects of the internal structure of the scalar field in liquid-phase transverse jets. In particular, the issue of local anisotropy is addressed with two-dimensional power spectra, scalar microscales and scalar increments in two directions. An attempt is made to quantify the observed smallscale anisotropy and identify its cause. Based on an observed correlation between the small-scale anisotropy and the mean strain field, it is suggested that the large-scale vortex dynamics of the transverse jet are responsible for the local scalar anisotropy in the far field $(\S 5)$.

\section{Experiments and visualization}

Experiments on high-Schmidt-number turbulent mixing in the transverse jet were conducted in the GALCIT free-surface water tunnel (FSWT), a closed-circuit facility having a $50.8 \mathrm{~cm}$ wide $\times 76.2 \mathrm{~cm}$ deep (20 in. $\times 30$ in.) test section. The FSWT was operated as a water tunnel with square cross-section for these experiments by fitting a surface plate at the free-surface; for all experiments, the water level in the test section was maintained at a depth of $50.8 \mathrm{~cm}$ (20in). A neutrally buoyant jet of water was injected downward into the crossflow from the top of the surface plate (figure 1). Flow was initiated by pressurizing an inverted liquid-filled plenum with air; the jet flowed out of a nozzle block that was fitted flush to the surface plate. The jet nozzle was convex contoured to suppress formation of Görtler vortices and had an area-contraction ratio of 43 . The nozzle was $31.8 \mathrm{~mm}(1.25 \mathrm{in})$ long and had an internal exit diameter of $7.62 \mathrm{~mm}(0.300 \mathrm{in})$. Once filled, the plenum was allowed to settle for at least $30 \mathrm{~min}$ before each run to allow flow disturbances to die down. 


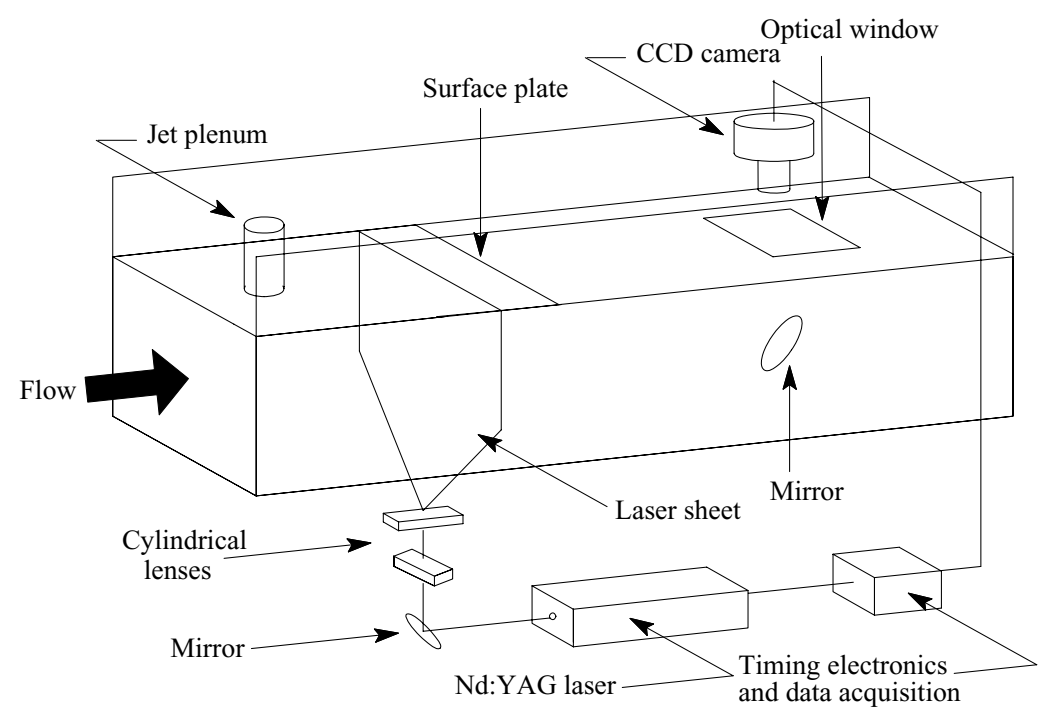

FIGURE 2. Imaging configuration for perpendicular cross-sections of transverse jet at $x / d_{j}=50$.

Experiments were conducted at fixed jet-to-free-stream velocity ratio $V_{r} \equiv U_{j} / U_{\infty}=$ 10 for several Reynolds numbers. Selected experiments were performed at a higher velocity ratio, $V_{r}=32$, for visualization purposes, but all quantitative data discussed in this paper came from jets at $V_{r}=10$. Particular attention was paid to the transverse jet at $V_{r}=10$ because that velocity ratio was identified as a critical ratio for 'strong' jets, in the sense that wall (pressure) effects on the jet trajectory can be neglected in favour of entrainment effects for $V_{r} \geqslant 10$. Hasselbrink \& Mungal (2001) show that the similarity analysis of Broadwell \& Breidenthal (1984), which predicts the well-known power-law trajectory for transverse jets, implicitly assumes that $V_{r} \geqslant 10$. In addition, the $V_{r}=10$ jet is of interest because that velocity ratio has been identified as a critical ratio for the appearance of jet fluid in the wake; higher velocity-ratio jets were observed to have jet fluid in the wake, while lower velocity ratio jets did not (Smith \& Mungal 1998). Velocity ratios between 10 and 20, depending on the assumed stoichiometry, have also been reported to result in minimum flame lengths (Broadwell \& Breidenthal 1984; Smith \& Mungal 1998).

For all but some limited visualizations, the velocity ratio was thus maintained constant at $V_{r}=10$ while the jet Reynolds number was varied in the range $1.0 \times 10^{3} \leqslant R e_{j} \equiv U_{j} d_{j} / v \leqslant 20 \times 10^{3}$ by suitably adjusting the jet and free-stream velocities. Streamwise slices of the jet were taken at $R e_{j}=1.0,2.0,5.0$ and $10 \times 10^{3}$, whereas transverse slices were taken at $x / d_{j}=50$ for $R e_{j}=1.0,2.0,5.0,10$ and $20 \times 10^{3}$. The crossflow boundary layer (the boundary layer on the surface plate) was laminar and thin, calculated to be between $0.067 d_{j}$ and $0.30 d_{j}$ at the jet exit, depending on Reynolds number. Table 1 summarizes the experimental conditions for the primary case of interest, the transverse slices at $x / d_{j}=50$.

\subsection{Imaging technique}

Digital imaging of laser-induced fluorescence (LIF) was used to measure mixed-fluid concentration fields (Walker 1987) in the transverse jet (figure 2). The jet plenum was filled with a dilute mixture of rhodamine-6G chloride and water, in molar concentrations of $1.4 \times 10^{-6} \mathrm{M}$ (streamwise views) and $1.4 \times 10^{-5} \mathrm{M}$ (transverse views). 


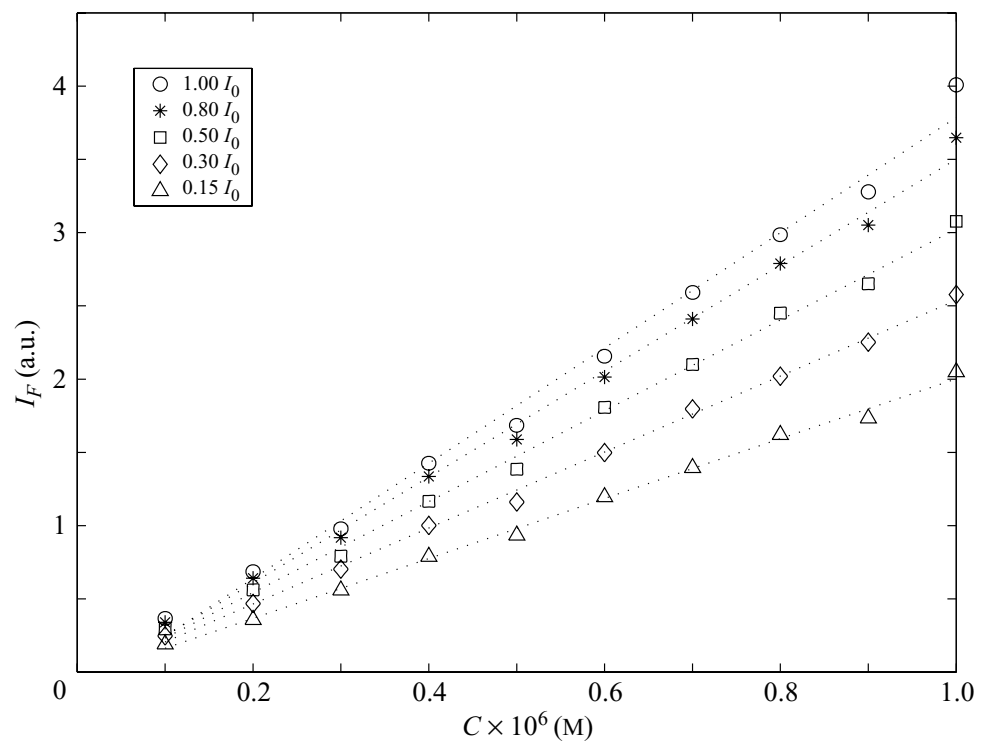

FIGURE 3. Variation of fluorescence intensity with dye concentration, over the indicated range of laser intensities. $I_{0}=10^{11} \mathrm{~W} \mathrm{~m}^{-2}$.

Upon excitation at $\lambda_{L}=532 \mathrm{~nm}$ with a frequency-doubled Q-switched Nd:YAG laser (Continuum YG661), the dyed jet fluid fluoresced at $\lambda_{F}=555 \mathrm{~nm}$ while the undyed tunnel fluid remained dark (e.g. Pringsheim 1949). The laser provided $250 \mathrm{~mJ} \mathrm{pulse}^{-1}$ (in the green) with a pulse duration of approximately 6 ns. An optical low-pass filter (Kodak no. 21) was used to isolate the fluorescence emission from the laser wavelength spectrally. Experiments were performed in a darkened laboratory to further minimize noise from ambient light.

The suitability of rhodamine-6G dye and a high-powered pulsed Nd:YAG laser for quantitative measurement of the scalar field was verified in separate experiments (Shan, Lang \& Dimotakis 2004). To summarize, the fluorescence intensities, $I_{F}$, of various rhodamine-6G solutions were measured in a cuvette for concentrations and laser intensities similar to those of the jet-imaging experiment. The fluorescence of aqueous solutions of rhodamine-6G (at similar concentrations to those occurring in the present experiments) was found to be approaching saturation owing to the high instantaneous power of the pulsed laser. Although the fluorescence was not linear with illumination intensity, the fluorescence was nonetheless linearly proportional to concentration (figure 3). This enabled quantitative measurement of the jet-fluid concentration field, using the procedure described below.

Because of the linearity of fluorescence with concentration, the imaged intensity of fluorescence, $I_{F}\left(x_{1}, x_{2}, t\right)$, of a time-varying concentration field, $c\left(x_{1}, x_{2}, t\right)$, can be written as,

$$
I_{F}\left(x_{1}, x_{2}, t\right)=g\left[I_{L}\left(x_{1}, x_{2}\right), S\left(x_{1}, x_{2}\right)\right] c\left(x_{1}, x_{2}, t\right)+I_{b a c k}\left(x_{1}, x_{2}\right),
$$

where $g\left(I_{L}, S\right)$ is an undetermined function of the local laser intensity, $I_{L}\left(x_{1}, x_{2}\right)$ and the pixel-by-pixel sensitivity, $S\left(x_{1}, x_{2}\right)$, of the imaging system. $I_{\text {back }}$ is the cumulative background level due to dark noise, offsets, etc. in the CCD camera. The imaged intensity for a reference, uniform-concentration field would be,

$$
I_{F, r e f}\left(x_{1}, x_{2}\right)=g\left[I_{L}\left(x_{1}, x_{2}\right), S\left(x_{1}, x_{2}\right)\right] c_{r e f}+I_{\text {back }}\left(x_{1}, x_{2}\right),
$$


where $c_{\text {ref }}$ is a known concentration. The laser-illumination fields for the jet image, $(2 a)$, and the reference image, $(2 b)$, are the same, or nearly so, because the laser attenuation due to absorption is negligible. $\dagger$ In this case then, the mixed-fluid concentration field, referenced to the known concentration, may be computed by subtracting the background-illumination images and normalizing by the uniformconcentration images (cf. $(2 a)$ and $(2 b))$, i.e.

$$
\frac{c\left(x_{1}, x_{2}, t\right)}{c_{\text {ref }}}=\frac{I_{F}\left(x_{1}, x_{2}, t\right)-\left\langle I_{\text {back }}\left(x_{1}, x_{2}\right)\right\rangle}{\left\langle I_{F, \text { ref }}\left(x_{1}, x_{2}\right)\right\rangle-\left\langle I_{\text {back }}\left(x_{1}, x_{2}\right)\right\rangle} .
$$

This result relies on linearity with respect to concentration, as demonstrated in figure 3 , but is independent of the precise functional form of $g(I, S)$. Thus, the ability to make quantitative measurements of scalar concentration using pulsed-laser LIF depends only on weak absorption and the linearity of fluorescence with concentration, which were both verified for the present experiments.

Fluorescence images of the mixed-fluid concentration field were recorded in both streamwise (figure 1) and perpendicular (figure 2) cross-sectional views of the transverse jet. For both cases, a laser sheet was formed in order to illuminate a thin slice of the jet-fluid concentration field. A negative-focal-length cylindrical lens expanded the beam into a laser sheet, while a long-focal-length cylindrical lens focused the sheet to a thin waist centred in the field of view. The sheet was $0.19 \mathrm{~mm}$ thick at its waist, and had a Rayleigh range of $20 \mathrm{~cm}$. Images of streamwise cross-sections of the jet were recorded on the centreline for a square field of view of dimensions $40 \mathrm{~cm}(16 \mathrm{in}$ or $53 d_{j}$ ) on a side. Images of transverse cross-sections were recorded for a field of view of $24 \mathrm{~cm}\left(9.3\right.$ in or $\left.31 d_{j}\right)$ on a side, at a downstream location of $x / d_{j}=50$. The pixel (in-plane) resolution in transverse images was $0.23 \mathrm{~mm}$, which is comparable to the laser-sheet thickness of $0.19 \mathrm{~mm}$. The pixel resolution in the streamwise images was $0.40 \mathrm{~mm}$. Additional discussion of the resolution of the experiment is given in $\S 2.3$.

As shown in figures 4-7, the coordinate system is taken to have its origin at the jet exit, with $y$ in the direction of jet injection (downward), $x$ in the crossflow direction, and $z$ in the spanwise direction.

A digital camera was custom-designed and constructed to record the images of the mixed-fluid concentration field. The camera used a low-noise high-dynamic-range CCD image detector developed by NASA's Jet Propulsion Laboratory for the Cassini spacecraft. This 'Cassini' imaging system was capable of true 12-bit dynamic range at $(1024 \times 1024)$-pixel resolution at a framing rate of $10 \mathrm{~Hz}$. Sequences of 508 images were recorded for perpendicular cross-sections of the jet, and 254 images for streamwise cross-sections.

Background images, $I_{b a c k}\left(x_{1}, x_{2}\right)$, were recorded shortly before each run, with the laser firing and an optical low-pass filter on the camera lens, but without starting the fluorescent-dye-seeded jet. Then, after the jet was run, reference images, $I_{F, \text { ref }}\left(x_{1}, x_{2}\right)$, of a uniform concentration field were recorded by immersing a transparent acrylic container filled with well-mixed dye of known concentration, $c_{r e f}$, in the test section. Using these background and reference images, the effects of CCD sensitivity variation, illumination non-uniformity, and optical transfer function were removed using (3). The imaged jet-fluid concentration was referenced to the jet-plenum dye concentration, $c_{0}$,

$\dagger$ The low concentrations used in the jet experiment, and near-saturation of fluorescence, caused absorption to be a small fraction of the initial laser intensity, $I_{L, 0}$ (Shan et al.2004). It was verified in fluorescence measurements of a uniform-concentration field that laser attenuation due to absorption was small for the mean-concentration-pathlength of the jet images. 


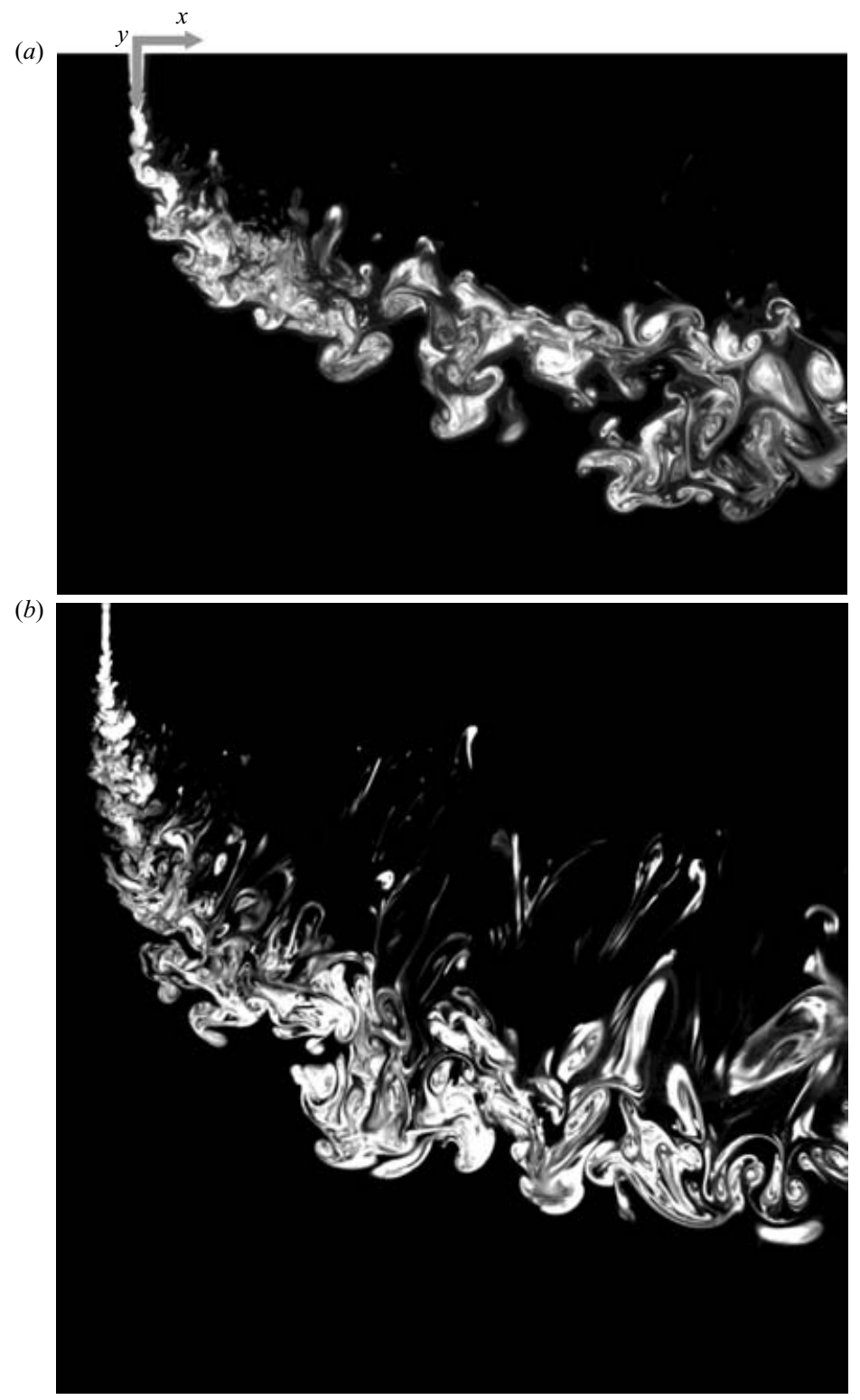

FIGURE 4. Mixed-fluid concentration in a streamwise cross-section of the transverse jet at $R e_{j}=1.0 \times 10^{3}$. The intensity of the image shown has scaled by $x^{1 / 2}$ to compensate for downstream decay. (a) $V_{r} \simeq 10$. (b) $V_{r} \simeq 32$.

by scaling by $c_{r e f} / c_{0}$, which is known a priori. This ultimately yields the mixed-fluidconcentration values, normalized to the plenum concentration, so that

$$
0 \leqslant C\left(x_{1}, x_{2}, t\right) \equiv \frac{c\left(x_{1}, x_{2}, t\right)}{c_{0}}=\frac{c\left(x_{1}, x_{2}, t\right)}{c_{r e f}} \frac{c_{r e f}}{c_{0}} \leqslant 1 .
$$

In this way, the scalar-field measurements were normalized so that $C=1$ corresponds to unmixed jet fluid (at the jet exit) and $C=0$ corresponds to pure crossflow (free-stream) fluid. 


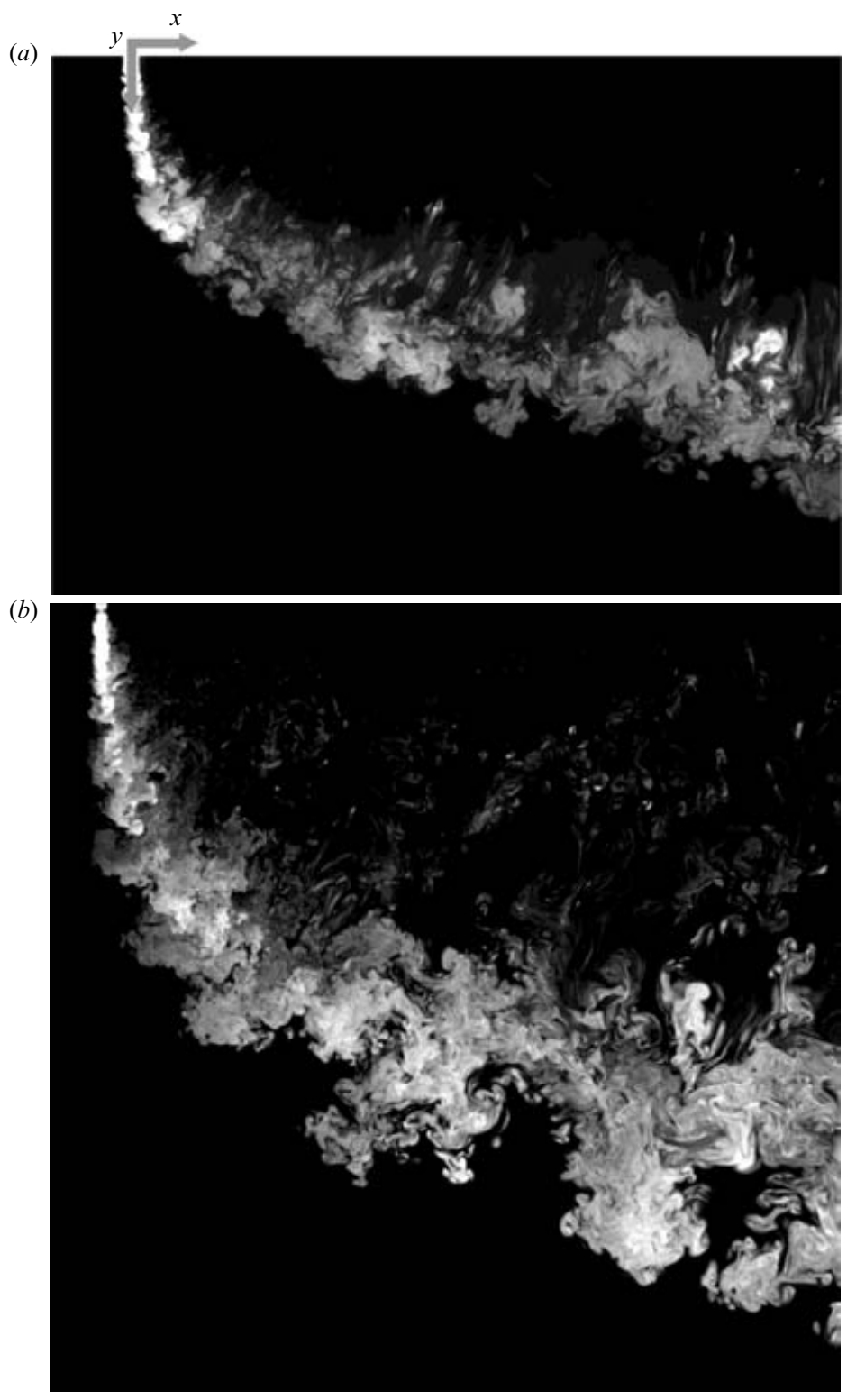

FIGURE 5. As for figure 4, but $R e_{j}=10 \times 10^{4}$.

For streamwise slices of the jet, any possible shot-to-shot variations in the power of the pulsed laser were measured and normalized by monitoring the fluorescenceintensity fluctuations at the jet exit. This was made possible by the fact that the jet exit remained visible in the streamwise images, and that the jet-exit concentration never varied. For transverse slices of the jet, the jet exit was no longer visible and an alternative reference cell was required. In that case, a fibre-optic probe was used to deliver a fraction of the laser output to a test tube containing a small sample of fluorescent dye. An image of the test-tube fluorescence was recorded on an unused corner of the CCD array and used to detect and normalize any shot-toshot power fluctuations of the laser. In other LIF experiments, which typically use 


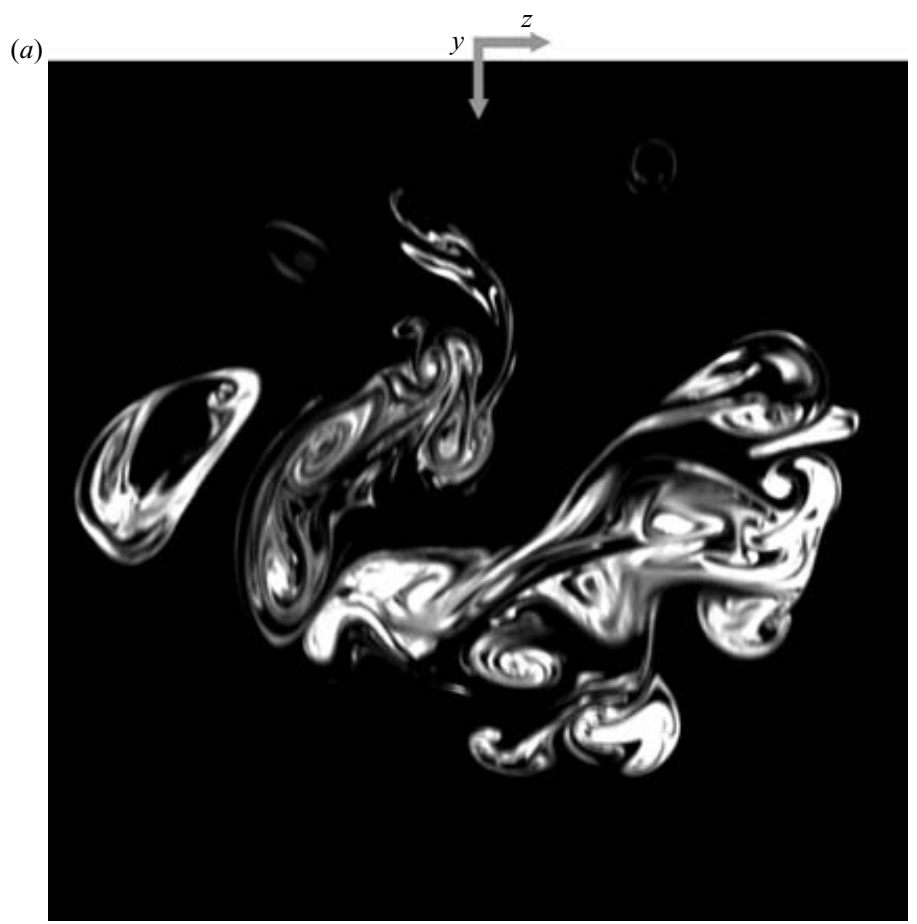

(b)

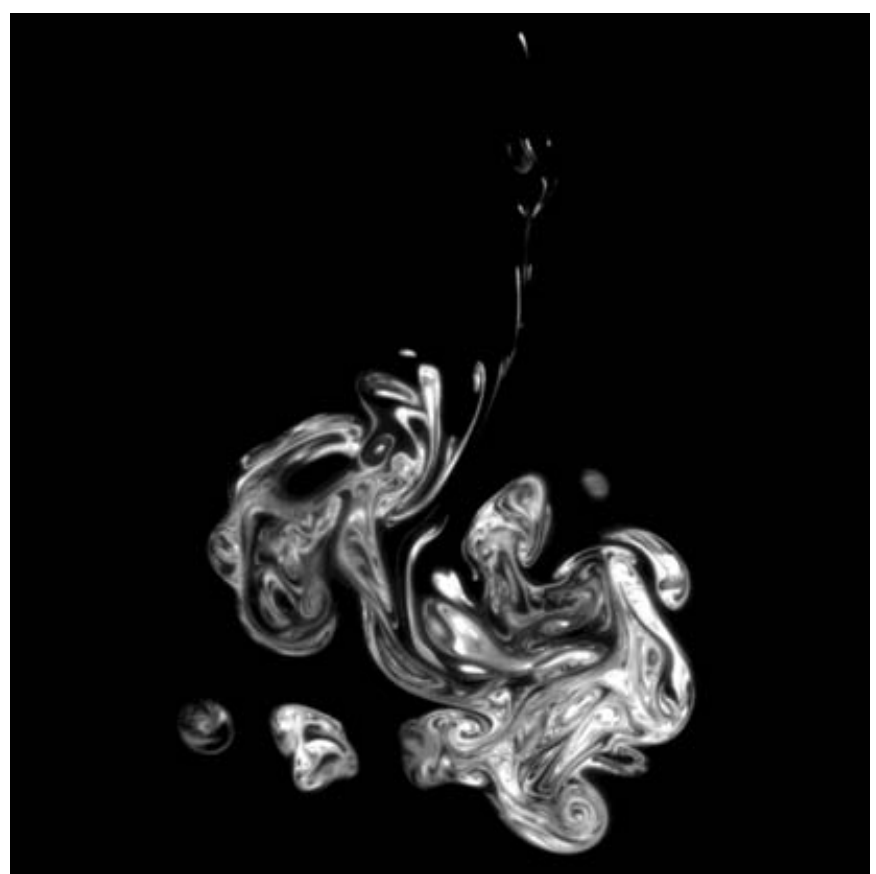

FIGURE 6. Mixed-fluid concentration in a perpendicular cross-section of the jet at $R e_{j}=1.0 \times 10^{3}$. (a) $V_{r} \simeq 10$ and $x / d_{j}=50$. (b) $V_{r} \simeq 32$.

continuous-wave lasers, previous investigators have used reference cells in a similar fashion to account for temporal variations, attenuation and other non-idealities in the optical system (e.g. Koochesfahani \& Dimotakis 1986; Dahm \& Dimotakis 1990). 


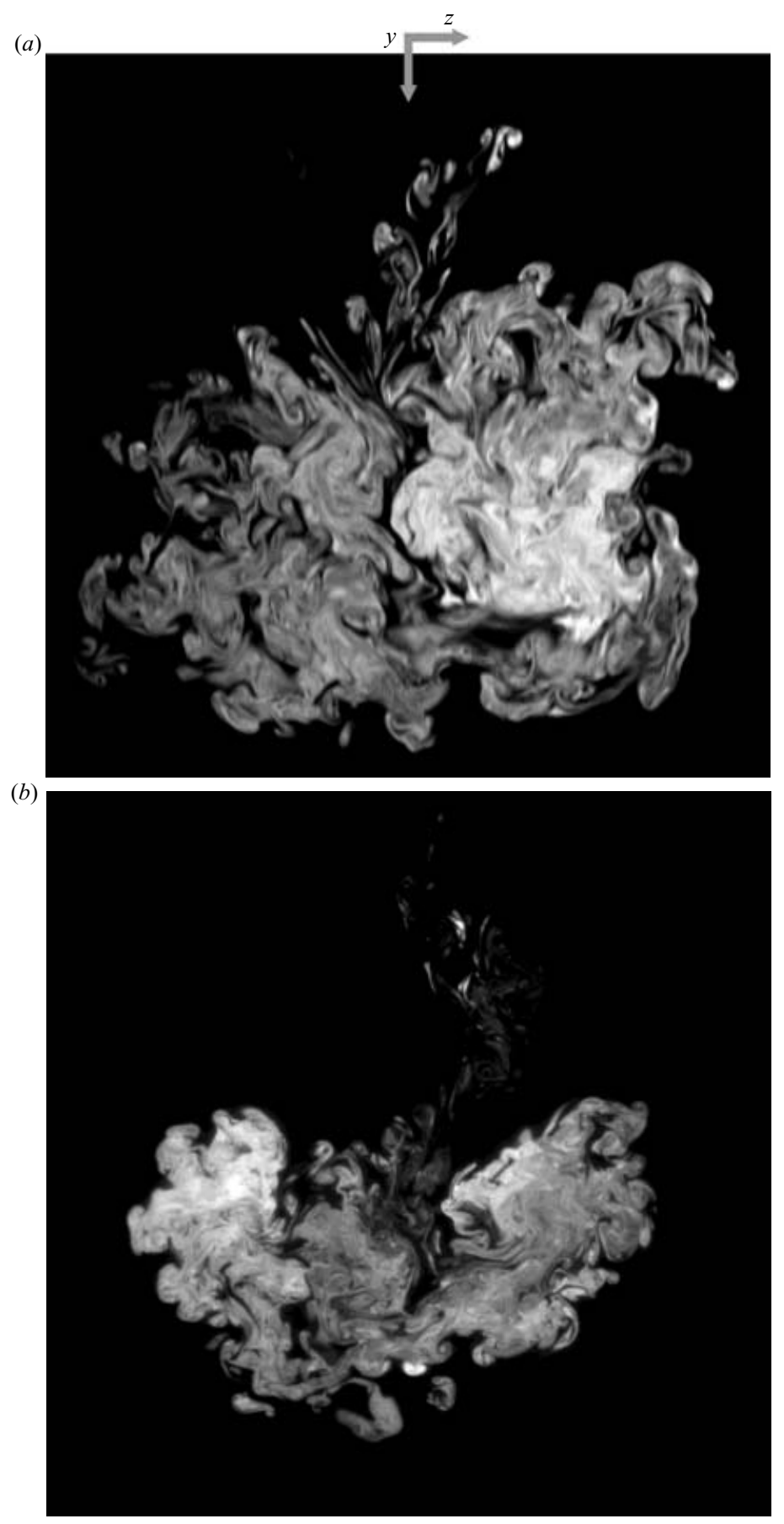

Figure 7. As figure 6, but $R e_{j}=10 \times 10^{3}$.

Figures 4 and 5 show examples of streamwise cross-sectional images at Reynolds numbers of $1.0 \times 10^{3}$ and $10 \times 10^{3}$, respectively. (For display purposes only, the images shown in figures 4 and 5 have been scaled to compensate for the downstream decay 
in concentration and image intensity. The images intensity was adjusted by a factor proportional to the square root of the downstream distance (i.e. $x^{1 / 2}$ ) starting just upstream of the jet exit. This scaling, of course, was not applied to the data used for quantitative analysis.) The upper images are for a jet-to-crossflow velocity ratio of 10 , and the lower images for a velocity ratio of 32 . In the low-Reynolds-number case, the jet core remained laminar for several $d_{j}$ after exiting the nozzle; however, at the higher Reynolds number, the jet's potential core transitioned and mixed within approximately one $d_{j}$. From the images, it can also been seen that the transverse jet's scalar field has very small-scale structure at its upper half (the 'wake' region); the jet is noticeably smoother on the bottom. Also clearly visible on the jet centreline at $V_{r}=32$, and still present but less noticeable at $V_{r}=10$, are the filaments ('hairs' or 'fingers') of jet fluid extending upward from the jet body toward the injection wall. These thin filaments have a preferred orientation in the vertical direction, and contribute to the local anisotropy of the scalar field, as will be discussed in $\S 5$.

Figures 6 and 7 show examples of transverse cross-sections of the jet at $R e_{j}=10^{3}$ and $R e_{j}=10 \times 10^{3}$, respectively. A kidney-shaped structure is seen which is associated with a counter-rotating vortex pair. From a time sequence of images, the jet position and the size of the scalar 'lobes' can be seen to vary over time. As was the case with the streamwise view of the jet, thin vertical filaments can be seen in the wake region; these filaments extend from the middle of the counter-rotating vortex pair up toward the injection wall. These fine-scale features in the wake region are more apparent in the transverse slices than in the streamwise slices because, in the cross-sectional views, they are captured even if they do not fall precisely on the jet centreline.

The appearance of jet fluid in the wake, which is seen from the data for $V_{r} \geqslant 10$, has been previously reported by Lozano et al.(1993) and Smith \& Mungal (1998) to occur at velocity ratios between 10 and 15. Fric and Roshko (1994) concluded, based on the absence of jet fluid in the wake for high velocity ratios, that the boundary layer on the plate (i.e., the crossflow boundary layer), and not the jet boundary layer (originating within the jet nozzle), was the source of vorticity for the organized vortices that they observed in the wake region of the transverse jet. The fact that jet fluid is observed in the wake for $V_{r} \geqslant 10$ implies that Fric \& Roshko's reasoning does not apply for high-velocity-ratio jets; their conclusion may still be correct, however. Smith \& Mungal (1998) have proposed that wake vortices, when stretched, could have strong axial flows that would pull fluid from the jet into the wake region. This explanation would reconcile the observation that the jet fluid appears in the wake with Fric \& Roshko's proposal that the wake vortices arise from the crossflow boundary layer. However, the observed presence of jet fluid in the wake means that it cannot be completely ruled out based on existing arguments that the vorticity in the wake region arises from a source other than the crossflow boundary layer.

\subsection{Local Reynolds number and circulation}

Unlike the axisymmetric jet discharging in a quiescent reservoir, the local Reynolds number of the jet in crossflow changes with increasing downstream distance. It is shown in the following that the local Reynolds number, $R e_{\Gamma}$, based on the circulation of a counter-rotatating vortex pair, decays in power-law fashion with downstream distance.

Following the analysis of Broadwell \& Breidenthal (1984), a jet is considered which discharges perpendicularly at velocity $U_{j}$ into a crossflow of velocity $U_{\infty}$. The jet becomes a point source of momentum in the limit in which the jet momentum flux, $\dot{m}_{j} U_{j}=\rho_{j} \pi\left(d_{j} / 2\right)^{2} U_{j}^{2}$, is held constant as jet diameter, $d_{j}$, decreases, and the discharge 
velocity, $U_{j}$, increases. This point source of normal momentum, i.e. a 'lift' force of vanishing drag, generates a counter-rotating vortex pair that is analogous to the tip-vortices behind a finite-span wing generating lift. Broadwell \& Breidenthal argue that viscosity has no global role but only serves to dissipate energy at the small scales; thus, the only global length scale possible from dimensional analysis is:

$$
l=\left(\frac{\dot{m}_{j} U_{j}}{\rho_{\infty} U_{\infty}^{2}}\right)^{1 / 2},
$$

For the case of equal density fluids, as is the case in the present experiments, the global length scale reduces to $l=(\pi / 4)^{1 / 2} d_{j} V_{r}$.

The circulation, $\Gamma$, of one vortex of the vortex pair is related to the vortex-core separation distance, $R$, and the vortices' vertical velocity, $\mathrm{d} y / \mathrm{d} t$ :

$$
\frac{\mathrm{d} y}{\mathrm{~d} t}=\frac{c_{1} \Gamma}{R} .
$$

Also, the fluid impulse per unit length, $P$, of the vortex is:

$$
P=c_{2} \rho_{\infty} \Gamma R .
$$

The values of the constants $c_{1}$ and $c_{2}$ in (5) and (6) depend on the spatial distribution of vorticity; for example, $c_{1}=\pi / 4$ and $c_{2}=2$ for ideal line vortices. It should be noted that (5) and (6) assume that the jet takes the form of a single counter-rotating vortex pair. (If the circulation is divided between more than two vortices, each vortex could have different induced velocities, $\mathrm{d} y / \mathrm{d} t$, and differing trajectories. The basic case, in which a single vortex pair is found, is considered for this discussion.) A similarity form is assumed in which the flow is independent of the global length scale $l$ in the far field, so that $R$ is proportional to $y$. Then, (5) and (6) may be combined to eliminate $\Gamma$, and the result integrated, assuming $R \propto y$, to find the trajectory:

$$
y=c_{3}\left(\frac{P}{\rho_{\infty}}\right)^{1 / 3} t^{1 / 3} .
$$

With the far-field transformation $x=U_{\infty} t$, and noting that $P=\dot{m}_{j} U_{j} / U_{\infty}$, the trajectory of the transverse jet is,

$$
\frac{y}{l}=c_{3}\left(\frac{x}{l}\right)^{1 / 3},
$$

as found by Broadwell \& Breidenthal (1984).

It can be seen from the preceding equations that the circulation of each vortex in the transverse-jet decreases with downstream distance, $x$, as:

$$
\frac{\Gamma}{U_{\infty} l}=c_{4}\left(\frac{x}{l}\right)^{-1 / 3} .
$$

Equation (9) can be expected to hold in the far field of high-velocity ratio transverse jets, in particular, for $x>l$, where $l \equiv V_{r} d_{j}$. An alternative definition of the far field has been proposed by Smith \& Mungal (1998) based upon centreline concentration decay measurements. By their measure, the far field begins for $s / d_{j}>0.3 V_{r}^{2}$, where $s$ is the arclength along the jet trajectory. Regardless of which definition is used, the measurements of jet cross-sections at $x / d_{j}=50$ that are described in the following sections are in the far field.

It should also be noted that the decreasing circulation of the vortex pair (equation (9)) is not a consequence of viscous decay of vorticity because the analysis 


\begin{tabular}{ccccrcc}
$R e_{j}$ & $V_{r}$ & $\Theta / d_{j}$ & \multicolumn{1}{c}{$\operatorname{Re}_{\Gamma}$} & $R e_{\lambda}$ & $\lambda_{v} / \lambda_{p}$ & $\lambda_{\mathscr{D}} / \lambda_{p}$ \\
$1.0 \times 10^{3}$ & 10 & 0.30 & $0.58 \times 10^{3}$ & 32 & 281 & 5.3 \\
$2.0 \times 10^{3}$ & 10 & 0.21 & $1.2 \times 10^{3}$ & 45 & 167 & 3.2 \\
$5.0 \times 10^{3}$ & 10 & 0.13 & $2.9 \times 10^{3}$ & 71 & 84 & 1.6 \\
$10 \times 10^{3}$ & 10 & 0.094 & $5.8 \times 10^{3}$ & 100 & 50 & 0.94 \\
$20 \times 10^{3}$ & 10 & 0.067 & $12 \times 10^{3}$ & 141 & 30 & 0.56
\end{tabular}

TABLE 1. Experimental conditions and imaging resolution of the LIF measurements at $x / d_{j}=50 . \lambda_{p}$ is the in-plane pixel resolution for the transverse slices. The momentum thickness, $\Theta$, is computed at jet exit. The circulation-based $\left(R e_{\Gamma}\right)$ and Taylor $\left(R e_{\lambda}\right)$ Reynolds numbers are computed at $x / d_{j}=50$.

assumes inviscid flow. Hasselbrink \& Mungal (2001) argue that the decrease in circulation is a result of the circulation integral being taken across a decreasing number of vortex lines as one moves downstream. Because the vorticity is not contained in a single vortex tube, circulation measured in transverse planes need not be conserved, even in the inviscid limit. (The actual circulation that would be measured in an experiment would be even less owing to the viscous decay of two opposite-signed vortices in proximity to one another.)

Based upon the circulation, a local Reynolds number for the transverse jet at any far-field downstream location $x>l$ can be defined,

$$
\operatorname{Re}_{\Gamma}(x) \equiv \frac{\Gamma}{v}=c_{4} \frac{U_{\infty} l}{v}\left(\frac{x}{l}\right)^{-1 / 3} .
$$

The local Reynolds number, $\operatorname{Re}_{\Gamma}(x)$, is related to the jet-exit Reynolds number, $R e_{j}$, as

$$
\operatorname{Re}_{\Gamma}(x)=c_{5} \frac{U_{j} d_{j}}{v}\left(\frac{x}{l}\right)^{-1 / 3}=c \operatorname{Re}_{j}\left(\frac{x / d_{j}}{V_{r}}\right)^{-1 / 3},
$$

where $c_{5}$ and $c$ are constants. The value of the constant, $c$, can be estimated by noting that the local Reynolds number, $\operatorname{Re}_{\Gamma}(x)$, should approach (or at least be of the same order of magnitude as) the jet-exit Reynolds number, $R e_{j}$, as one moves closer to the jet exit (i.e. $c$ is of order one so that $R e_{\Gamma}(x) / R e_{j}$ is of order one as $x / l \rightarrow 1$ ). It is reasonable then to define $c=1$, with the resulting expression for the local, circulation-based Reynolds number as:

$$
\operatorname{Re}_{\Gamma}(x)=\left(\frac{x / d_{j}}{V_{r}}\right)^{-1 / 3} \operatorname{Re}_{j} .
$$

Thus, at downstream location $x / d_{j}=50$, the jet-exit Reynolds number, $R e_{j}$, is related to the local (outer-scale) Reynolds number by a factor of 0.6 for $V_{r}=10$. Both Reynolds numbers are summarized in table 1 for the experimental conditions of this paper.

Finally, the Taylor Reynolds number, based upon the root-mean-squared fluctuation of one component of velocity and the Taylor microscale, $\lambda_{T}$, can be estimated as

$$
R e_{\lambda} \equiv \frac{u_{r m s} \lambda_{T}}{v} \simeq R e^{1 / 2}
$$

for high Reynolds number (e.g. Frisch 1995). For the present experiment, the highest Taylor Reynolds number attained is $R e_{\lambda} \simeq 141$ (table 1). The scalar-species equivalent of the Péclet number for this flow, $R e_{\lambda} S c$, is estimated to reach a maximum of $3.9 \times 10^{5}$. 
The proceeding discussion, which found the local Reynolds number for the transverse jet, has consequences for the dissipation scales of the current experiment. The smallest scales of the transverse jet, and the imaging resolution, are discussed in the following section.

\subsection{Dissipation scales and resolution}

The resolution requirement for scalar concentration measurement is set by the scalar diffusion scale, $\lambda_{\mathscr{D}}$, which is itself a multiple of the viscous scale, $\lambda_{v}$,

$$
\lambda_{\mathscr{D}} \simeq S c^{-1 / 2} \lambda_{v} .
$$

The Schmidt number, $S c$, is the ratio of kinematic viscosity to scalar species diffusivity. For high molecular-weight dyes in liquid-phase flows, the Schmidt number is much larger than unity and the finest features in the scalar field can be significantly smaller than those in the velocity or vorticity fields. (The Schmidt number for rhodamine-6G in water is $S c \simeq 2800$ (e.g. Xu \& Yeung 1997).)

To estimate the viscous scale, it is possible to take the scale at which turbulence spectra deviate at high wavenumbers from $-5 / 3$ power-law behaviour. Based upon data from a variety of flows (wakes, grid turbulence, boundary layers, jets, etc.) across a wide range of Reynolds numbers $\left(23 \leqslant R e_{\lambda} \leqslant 3180\right)$, Dimotakis (2000) has estimated the viscous scale to be 50 times larger than the Kolmogorov scale, $\lambda_{K}$, i.e.

$$
\lambda_{\nu} \simeq 50 \lambda_{K} .
$$

The Kolmogorov scale, $\lambda_{K}$, is in turn defined as

$$
\lambda_{K} \equiv\left(\frac{v^{3}}{\epsilon}\right)^{1 / 4} .
$$

When no measured values for the dissipation rate are available, an alternative approximation to the Kolmogorov scale can be used:

$$
\frac{\lambda_{K}}{\delta(x)} \simeq c_{6} \operatorname{Re}(x)^{-3 / 4} .
$$

The constant, $c_{6}$ is of order unity, based upon the energy-dissipation measurements of Freihe, Van Atta \& Gibson (1971) in turbulent jets (Dimotakis 2000).

For the transverse jet, the jet width is a sensible outer scale, and the Reynolds number can be taken to be the local circulation-based Reynolds number $\operatorname{Re}_{\Gamma}$. Based on the jet width and the circulation-based Reynolds number, the scalar diffusion scales for the conditions of the present experiments can be computed; these estimates of the required spatial resolution to fully resolve the flow are shown in table 1 . In the table, the scalar diffusion scale is compared to the measurement resolution, $\lambda_{p}$, which is determined by either the pixel resolution of the images, or the thickness of the laser sheet, whichever is greater. For the transverse cross-sectional images of the jet, the pixel image resolution was $0.23 \mathrm{~mm}$ and the laser-sheet thickness was $0.19 \mathrm{~mm}$. Thus, comparing the image resolution to the estimated scalar-diffusion scale, the LIF measurements of concentration are seen to be resolved for transverse slices at $x / d_{j}=50$ for Reynolds numbers up to $10 \times 10^{3}$, and not fully resolved for $R e_{j}=20 \times 10^{3}$.

The consequences of inadequate spatial resolution when making measurements of passive scalar fields were discussed by Breidenthal (1981) and Koochesfahani \& Dimotakis (1986). Koochesfahani \& Dimotakis found for liquid-phase mixing layers that inadequate resolution mimicked the qualitative effect of enhanced molecular 
diffusion. However, a ten-fold degradation of measurement resolution did not change the mean concentration and only increased the probability of finding mixed fluid by approximately $10 \%$ on the centreline of the mixing layer. Thus, while the marginal spatial resolution for the $R e_{j}=20 \times 10^{3}$ transverse jet may artificially sharpen the peak of the probability distribution, it is unlikely to change the measured distribution of jet fluid significantly. Moreover, the Reynolds-number effects seen in the flow $(\S 3)$ begin at Reynolds numbers which are well resolved, and thus are unlikely to result from degrading resolution.

\subsection{Space-time visualization}

The global structure of the jet can be visualized by stacking a contiguous time-resolved sequence of images of the jet. The transverse jet is particularly amenable to such spacetime visualizations because Taylor's hypothesis holds reasonably well in the far-field of this flow and can be used to transform between time and space coordinates. For the transverse jet, the streamwise velocity deficit (the velocity of the wake relative to the free-stream velocity), decays rapidly with downstream distance, so that the streamwise velocity is approximately the free-stream velocity at a far-downstream location, i.e. $U_{x} \simeq U_{\infty}$ for the far field (e.g. Mungal \& Lozano 1996; Yuan \& Street 1998). In this case, the space-time visualizations provide a reasonable approximation to the instantaneous three-dimensional jet. It should be noted, however, that the growth of the jet with downstream distance is not captured by the space-time data. In a sense, however, alternative information is recorded; the space-time data sets, while not instantaneous spatial data, are nonetheless a three-dimensional slice of the four-dimensional space-time domain of the unsteady turbulent jet. The space-time images that are presented here are similar in spirit to the visualizations of Mungal \& Lozano (1996) of burning plumes in crossflow. Their visualizations, taken from video films of burning Kuwaiti oil wells, are qualitative images of burning plumes, rather than the quantitative LIF slices of density-matched jets considered in the present work. Mungal \& Lozano find, based on assembled side views (i.e. streamwise views) of the jet, that the downstream side (the wake side) of the jet displays considerably more complexity than the upstream side of the jet. Yoda, Hesselink \& Mungal (1992) have provided similar volume renderings of a turbulent jet without crossflow.

Visualizations of level-sets of jet-fluid concentration, i.e. $C(\boldsymbol{x}, t)=C_{i s o}$, are of particular interest because the area of the level sets affects the overall rate of reaction that would occur if the two fluids contained chemically reacting species. In figures 8 and 9, sequences of instantaneous images are assembled into three-dimensional spacetime visualizations of the scalar jet at two Reynolds numbers, $R e_{j}=1.0 \times 10^{3}$ and $R e_{j}=2.0 \times 10^{3}$. Taylor's hypothesis is used to transform time into an approximate streamwise distance. The $C \simeq 0.006$ isosurface is highlighted for both cases by selecting only a small concentration range centred at that concentration to be opaque and reflecting.

From the space-time visualizations, the scalar structure of the transverse jet at both Reynolds numbers can again be seen to be dominated by a pair of kidneyshaped, counter-rotating vortices. A deep cleft separates the two main lobes of the counter-rotating vortices, and fine-scale filaments extend up from the cleft into the wake, as was also seen in the individual images (figure 6). The wake side of the jet has more complex smaller-scale features than the upstream side, as previously seen by Mungal \& Lozano (1996). Comparing the two Reynolds numbers, a small tertiary vortex is seen at $R e_{j}=1.0 \times 10^{3}$ which is not seen at $R e_{j}=2.0 \times 10^{3}$. Rotation of the tertiary vortex is evident in the spiral wrapping of thin scalar filaments. For the 


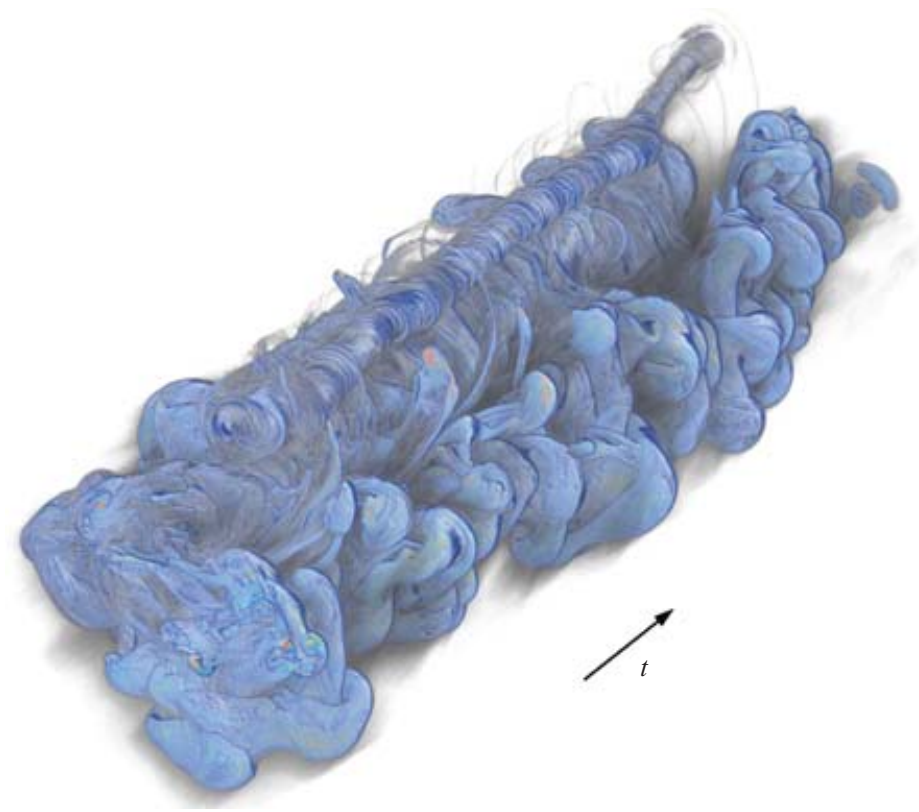

FIGURE 8. Space-time visualization of isosurface of $C \simeq 0.06$ for transverse jet at $R e_{j}=1.0 \times 10^{3}, V_{r}=10$, and $x / d_{j}=50$.

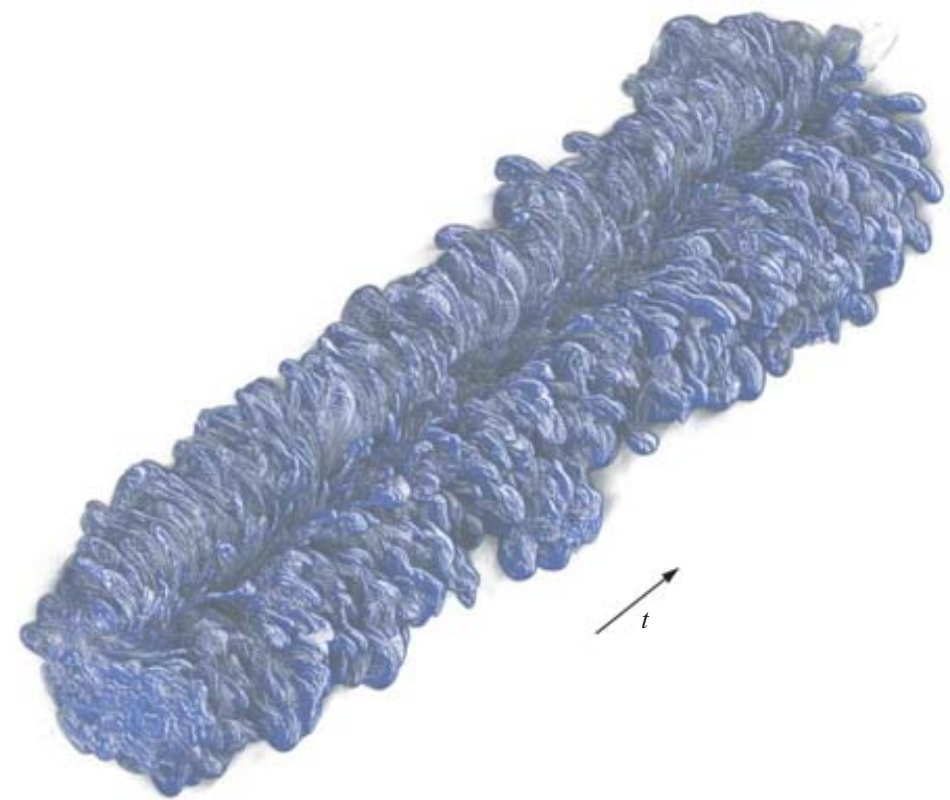

FIGURE 9. Space-time visualization of isosurface of $C \simeq 0.06$ for a $R e_{j}=2.0 \times 10^{3}, V_{r}=10$, transverse jet.

lower-Reynolds-number jet, the main lobes of the scalar field are not equal in size because of the jet fluid entrained in the tertiary vortex. Only two main lobes, approximately equal in size, are seen in the higher-Reynolds-number case, $R e_{j}=2.0 \times 10^{3}$. 
These limited visualizations are qualitatively consistent with the experiments of Kuzo (1995), which found a transition between asymmetric and symmetric counterrotating vortices in the transverse jet above a critical Reynolds number. Using particle image velocimetry to measure velocity and vorticity fields, Kuzo reported the existence of tertiary, and even quaternary vortices, at low Reynolds numbers. At $V_{r}=10$, the initially asymmetric jets appeared to transition to a symmetric flow state as the Reynolds number was increased above a critical value of $R e_{j} \simeq 6.0 \times 10^{3}$. Of course, the scalar-field measurements of the present study are not directly comparable to measurements of vorticity and velocity fields. However, the present data do indicate asymmetric structure and decreasing asymmetry with increasing Reynolds number in the flow-regime investigated. The general asymmetry of the transverse jet at downstream locations has been noted in experimental studies by Kamotani \& Greber (1972) and Smith \& Mungal (1998), among others. Smith and Mungal (1998) review the evidence for asymmetry in the transverse jet and conclude that it is quite common in experiments, although symmetry is often assumed in computational investigations.

\section{Reynolds-number effects and flow dependence}

By some measures, the behaviour of turbulent jets in crossflow appears to be insensitive to Reynolds number. Gross structural features, such as the jet size and trajectory, are insensitive to Reynolds number within the range that was investigated. Figure 10 shows two different 'sizes' of the jet cross-section at $x / d_{j}=50$. The size, $\delta_{\mathrm{h}}$, is defined as the horizontal extent of the jet for which the mean concentration is greater than a given percentage (either $3 \%$ or $5 \%$ ) of the peak concentration. As seen in the figure, the horizontal size is approximately independent of Reynolds number over the range $1.0 \times 10^{3} \leqslant R e_{j} \leqslant 20 \times 10^{3}$. Despite the different growth rates of the transverse jet and jet discharging in a quiescent reservoir, their sizes are comparable at $x / d_{j}=50$, where $\delta_{h} \simeq 23$ (cf. Dahm \& Dimotakis 1990). A further discussion of the relative entrainment rates of transverse jets and ordinary jets will be postponed until $\S 3.2$.

Reynolds number also appears to have little effect on jet trajectories. Mean jet trajectories, defined as the locus of points of maximum mean-concentration on the jet centreline, are shown in figure 11 for the same velocity ratio at two different Reynolds numbers, $R e_{j}=1.0 \times 10^{3}$ and $10 \times 10^{3}$. The trajectories essentially overlap, and both exhibit a power-law dependence of penetration depth on downstream distance. (For the higher-Reynolds-number jet, the trajectory dips slightly around $x / d_{j} \approx 10^{1.25}=18$ before continuing on at the same slope. The slight dip in jet trajectory is associated with a near-plateau that occurs in the mean concentration field at that location (see figure 11); a similar dip occurs at the same downstream location in the trajectory reported by Smith \& Mungal (1998, their figure 7) for the $V_{r}=$ 10 jet.) Early experiments by Pratte \& Baines (1967) found power-law trajectories with an exponent of 0.28 , and, as discussed in $\S 2$, subsequent modelling work by Broadwell \& Breidenthal (1984) predicted a power-law trajectory with an exponent of $1 / 3$. For comparison, lines of slope 0.28 and $1 / 3$ are shown in figure 11 . The trajectory of the present data is closer to that of Broadwell \& Breidenthal. It should be noted that the scalar trajectories are generally shallower than trajectories based on the maximum velocity or mean nozzle streamlines (e.g. Kamotani \& Greber 1972; Yuan, Street \& Ferziger 1999). Differences between scalar and velocity trajectories are also discussed by Su et al.(2000) and Hasselbrink \& Mungal (2001). 

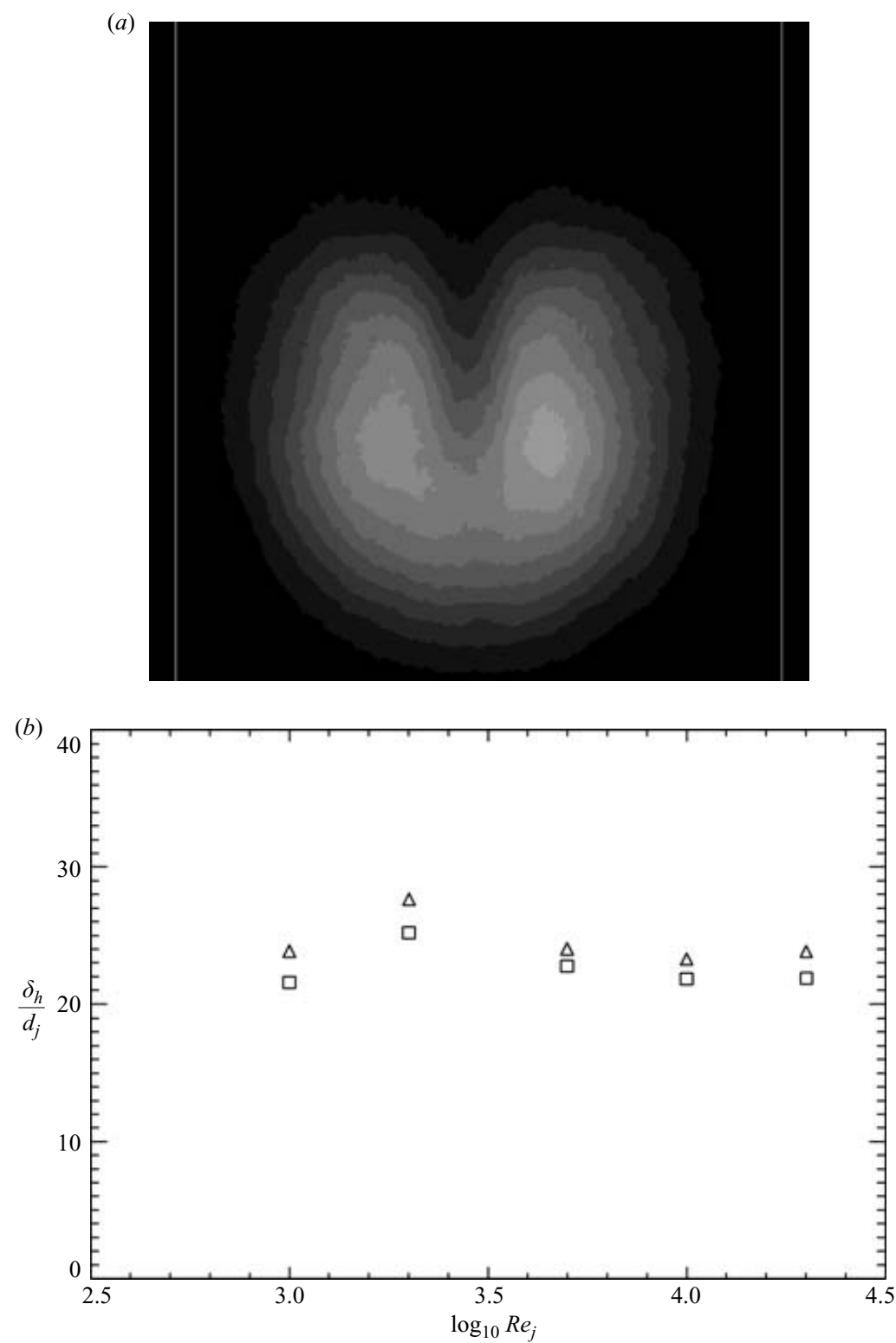

Figure 10. 'Size' of the $V_{r}=10$ transverse jet in cross-section, defined as the average horizontal extent, $\delta_{h}$, of mean scalar field at $x / d_{j}=50$. (a) Ensemble-averaged scalar field for $R e_{j}=20 \times 10^{3}$. Lines show horizontal extent of $3 \%$ of maximum mean-concentration. $(b)$ Size versus Reynolds number. $\triangle, 3 \%$ of maximum mean-concentration. $\square, 5 \%$ of maximum mean-concentration.

The decay of mean-concentration on the jet centreline is also insensitive to Reynolds number, over the range of values investigated. Figure 12 shows the centreline meanconcentration decay for $R e_{j}=1.0 \times 10^{3}$ and $R e_{j}=10 \times 10^{3}$. The lower-Reynoldsnumber jet has a longer potential core and initially decays more slowly with penetration depth (cf. figures 4 and 5). However, by about $x / d_{j} \simeq 6$, the decay of the low-Reynolds-number jet has caught up to that of the higher-Reynolds-number case. 
(a)
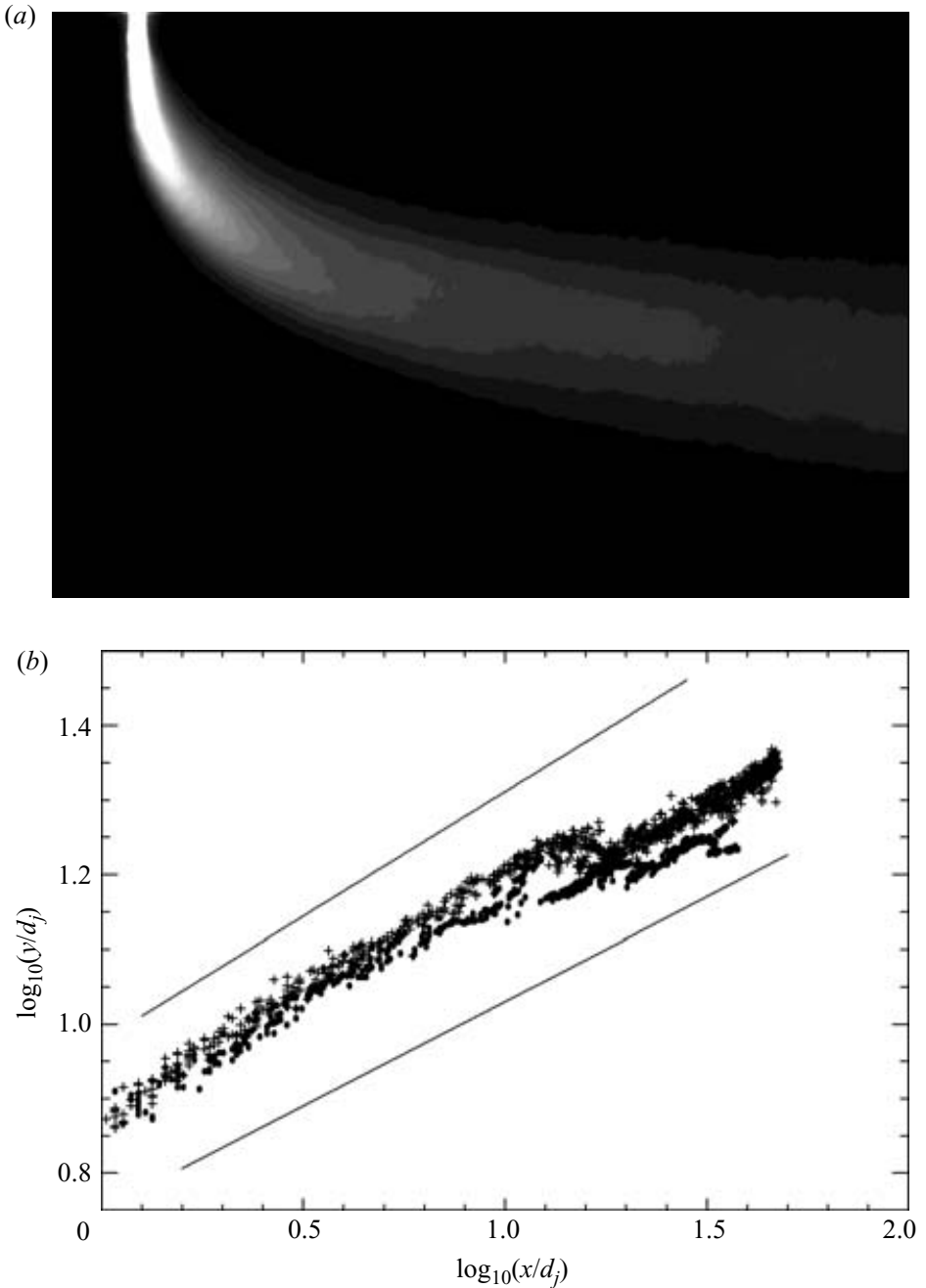

FiguRE 11. Mean concentration field and jet-centreline trajectory for $V_{r}=10$. (a) Ensemble-averaged concentration field for $R e_{j}=10 \times 10^{3}$. (b) Penetration depth versus downstream distance. Circles show $R e_{j}=1.0 \times 10^{3}$ and crosses show $R e_{j}=10 \times 10^{3}$. The lower comparison line is based on experiments of Pratte \& Baines (1967) and shows $y / d_{j} \propto\left(x / d_{j}\right)^{0.28}$. The upper comparison line is based on predictions of Broadwell \& Breidenthal (1984) and shows $y / d_{j} \propto\left(x / d_{j}\right)^{1 / 3}$.

Figure 13 depicts the concentration decay with downstream distance normalized in two ways, $x / d_{j}$ and $x / V_{r} d_{j}$. Apart from a difference due to potential-core lengths, the decay rates of the jet at the two Reynolds numbers are nearly indistinguishable.

As an aside, it should be noted that Smith \& Mungal (1998) reported a 'branch point' in the centreline concentration decay, representing, in their words, 'a transition in the flow field from enhanced mixing to reduced mixing compared to the free jet.' They identified the transition point as occurring around $s / V_{r}^{2} d_{j} \simeq 0.3$, or, approximately, $x / V_{r} d_{j} \simeq 1.6$ (Smith \& Mungal 1998, their figure 24). That location is marked in figure 13 with an arrow. For the $R e_{j}=10 \times 10^{3}$ case shown, a very slight change in the slope of the centreline concentration decay can be seen. While 


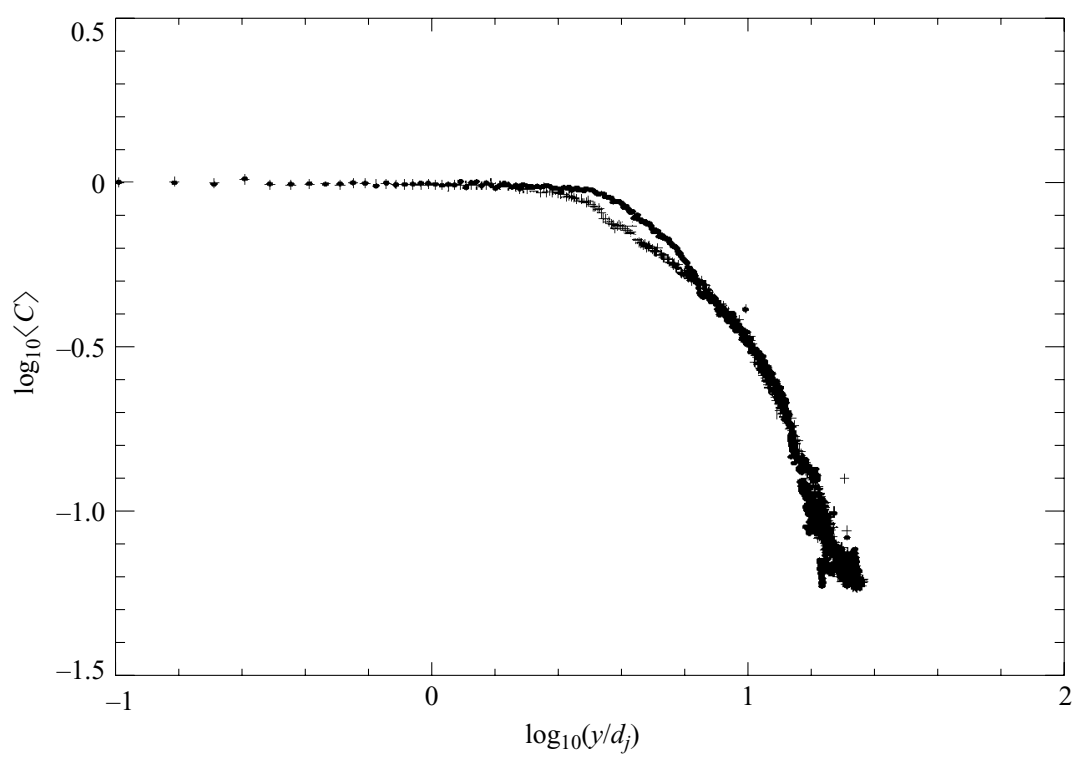

FIGURE 12. Decay of maximum mean-concentration with penetration depth at $V_{r}=10$. Small circles show $R e_{j}=1.0 \times 10^{3}$, and small crosses show $R e_{j}=10 \times 10^{3}$.

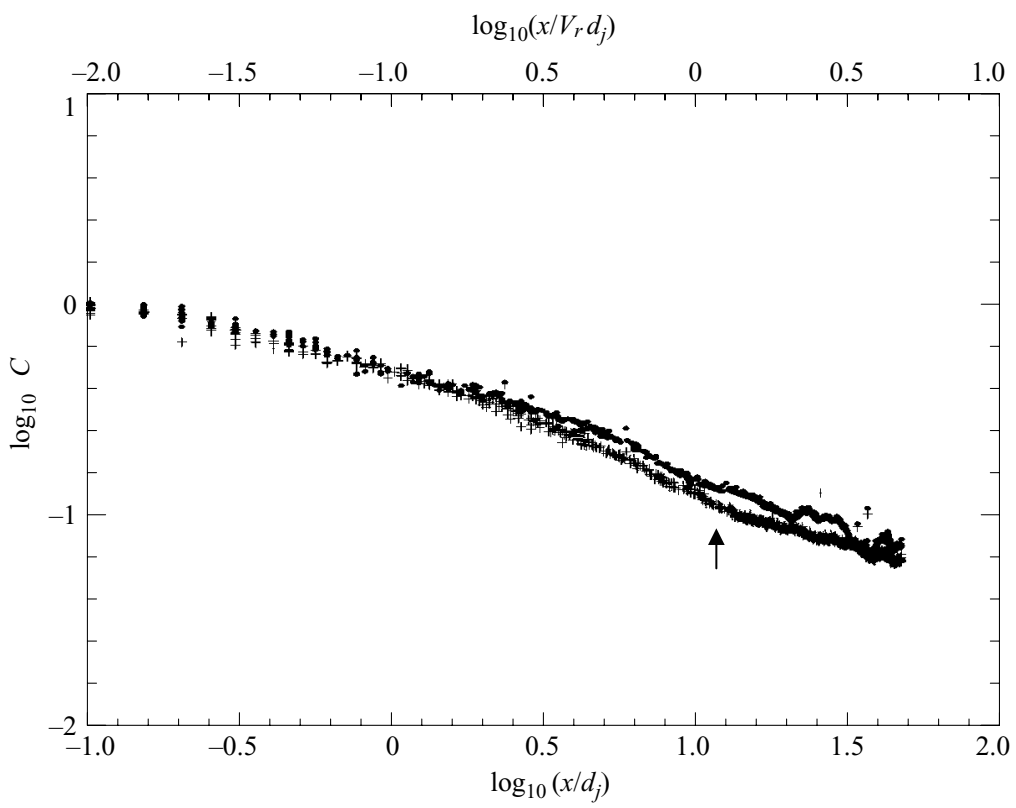

FIGURE 13. Decay of maximum mean-concentration with downstream distance at $V_{r}=10$. $R e_{j}=1.0 \times 10^{3}$ is shown with circles, $R e_{j}=10 \times 10^{3}$ with crosses. The top axis shows downstream distance normalized by $V_{r} d_{j}$, while the bottom axis shows downstream distance normalized by $d_{j}$. The arrow marks the location of a slight change in the slope of the decay reported by Smith \& Mungal (1998) at $\log \left(x / V_{r} d_{j}\right) \simeq 0.15$.

not conclusive, the present data are consistent with Smith \& Mungal's (1988) findings of a slight change in slope of the mean-concentration decay rate on the centreline of the transverse jet. 
However, it should be noted that mean quantities, such as mean-concentration decay rate or mean jet width, are not actual measures of mixing, but rather measures of entrainment. In particular, $\langle C\rangle$ is a measure of the ratio of jet fluid to total (jet fluid plus entrained crossflow fluid) mass flux. By definition, the mean concentration is the same for any probability distribution function having the same first moment. Thus, the mean cannot distinguish between perfectly homogenized fluids (one Dirac delta function), completely unmixed fluids (two Dirac delta functions), or intermediate states with the same first moment. To assess mixing, and its Reynolds-number dependence, measures other than mean quantities must be examined.

\subsection{Distribution of jet fluid}

A description of mixing involves knowledge of the probability density function of the conserved passive scalar, in this case, the jet-fluid concentration. The distribution, $f\left(C ; R e_{j}\right)$, of mixed-fluid concentration is normalized so that its definite integral is the probability that $C$ lies between the limits of integration:

$$
\operatorname{Pr}\left\{C_{1} \leqslant C<C_{2}\right\}=\int_{C_{1}}^{C_{2}} f(c) \mathrm{d} c=F\left(C_{1}\right)-F\left(C_{2}\right) .
$$

It should be noted that the distributions discussed in this paper are based on the probability of occurrence of a particular concentration $C$ in an instantaneous twodimensional image, rather than on the frequency of occurrence at a fixed point in space. Thus, they are spatial rather than temporal probability distributions. The spatial $\mathrm{PDF}$ is computed as the normalized magnitude of the differential area associated with various concentration values. In two dimensions, the PDF is (Kuznetsov \& Sabel'nikov 1990; Dimotakis \& Catrakis 1996),

$$
f(C)=\frac{1}{A_{t o t}}\left|\frac{\mathrm{d} A(C)}{\mathrm{d} C}\right|,
$$

where $A(C)$ is the area associated with a specified value of the scalar concentration, and $A_{\text {tot }}$ is the total area (domain) of the measured scalar field. Generalized to $d$-dimensions, the PDF would be computed as,

$$
f_{n}(C)=\frac{1}{V_{d, t o t}}\left|\frac{\mathrm{d} V_{d}(C)}{\mathrm{d} C}\right|,
$$

where $V_{d}(C)$ is the $d$-dimensional volume associated with $C$, and $V_{d, t o t}$ is the total, $d$ dimensional volume (domain) of the scalar field. Experimentally measured probabilitydensity functions depend in general on the dimensionality of the measurement, as well as on the form of the scalar field (see the Appendix). Thus, the PDFs described in the following discussion are more precisely the distribution of jet-fluid concentration in two-dimensional slices of the four-dimensional scalar field $C(x, y, z, t)$.

In contrast with structural measures such as the jet size and trajectory, or mean measures such as the decay of centreline concentration, the data indicate that the distribution of mixed fluid in the far field of a high-Schmidt-number strong transverse jet varies strongly with Reynolds number. The PDF of jet-fluid concentration at a fixed far-field location does not reach an asymptotic state, up to at least $R e_{j}=20 \times 10^{3}$. Figure 14 shows scalar PDFs for five different Reynolds numbers, $R e_{j}=1.0,2.0,5.0,10$ and $20 \times 10^{3}$. The measurement location was fixed at $x / d_{j}=50$ and the velocity ratio was maintained at $V_{r}=10$ in all cases. At the lowest Reynolds number, the PDF is qualitatively similar to the probability distribution of a twodimensional diffusive concentration field (derived in the Appendix). The distribution is 


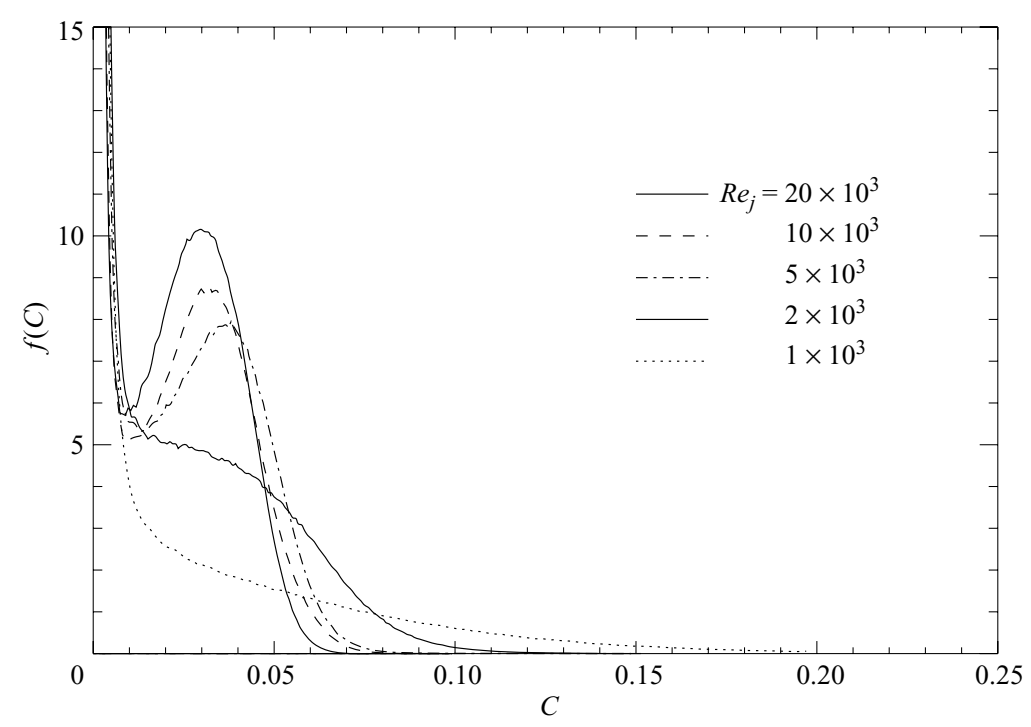

FIGURE 14. Distribution of jet-fluid-concentration for varying Reynolds number at $V_{r}=10$. Measurements made at $x / d_{j}=50$ for $R e_{j}=1.0,2.0,5.0,10$ and $20 \times 10^{3}$. Increasingly peaked PDFs with increasing Reynolds number.

broad, decreases monotonically, and peaks at very low jet-fluid concentration $(C \approx 0)$. As the Reynolds number is increased, the distribution of mixed fluid no longer decreases monotonically but develops a peak, i.e. there develops a most-probable concentration in the scalar field. The peak occurs by $R e_{j}=5.0 \times 10^{3}$. The preferredconcentration peak grows in height with increasing Reynolds-number up to the highest Reynolds numbers investigated, $R e_{j}=20 \times 10^{3}$. The scalar field is increasingly stirred and becomes more spatially homogeneous with increasing Reynolds number.

The image data themselves show additional evidence for a shift from a relatively unmixed to a well-mixed nearly homogeneous distribution with increasing Reynolds number. Figure 15 shows scalar fields for concentrations straddling the peak of the mixed-fluid PDF at high Reynolds numbers. Jet-fluid concentrations between $C=0.025$ and $C=0.045$ are highlighted in the image at two Reynolds numbers, $R e_{j}=1.0 \times 10^{3}$ and $R e_{j}=10 \times 10^{3}$. For the lower-Reynolds-number case, the concentration range, $0.035 \leqslant C \leqslant 0.045$, is confined to a thin region on the boundary of the jet. Everywhere else, the scalar field is relatively unmixed, with high concentration regions near the core of the jet and pure free-stream fluid outside. For the higher Reynolds-number jet, the specified concentration range is found throughout much of the body of the jet. The jet fluid is much more homogenously mixed owing to the turbulent stirring. Thus, the measured growth of PDF peaks with increasing Reynolds number is a direct consequence of the enhanced homogenization of the concentration field. It should also be noted that the PDF for each Reynolds number contains $5.3 \times 10^{8}$ individual measurements of the scalar field ( 508 frames at $(1024 \times 1024)$-pixel resolution). For the length of the recording, approximately four large-scale structure passages are captured at the lowest Reynolds number, and over 75 at the highest.

The most-probable concentration (i.e. the location of the PDF peak) and the width of PDF both vary with Reynolds number over the range $1.0 \times 10^{3} \leqslant R e_{j} \leqslant 20 \times 10^{3}$. Figure 16 shows the concentrations associated with the peaks of the jet-fluid 
(a)

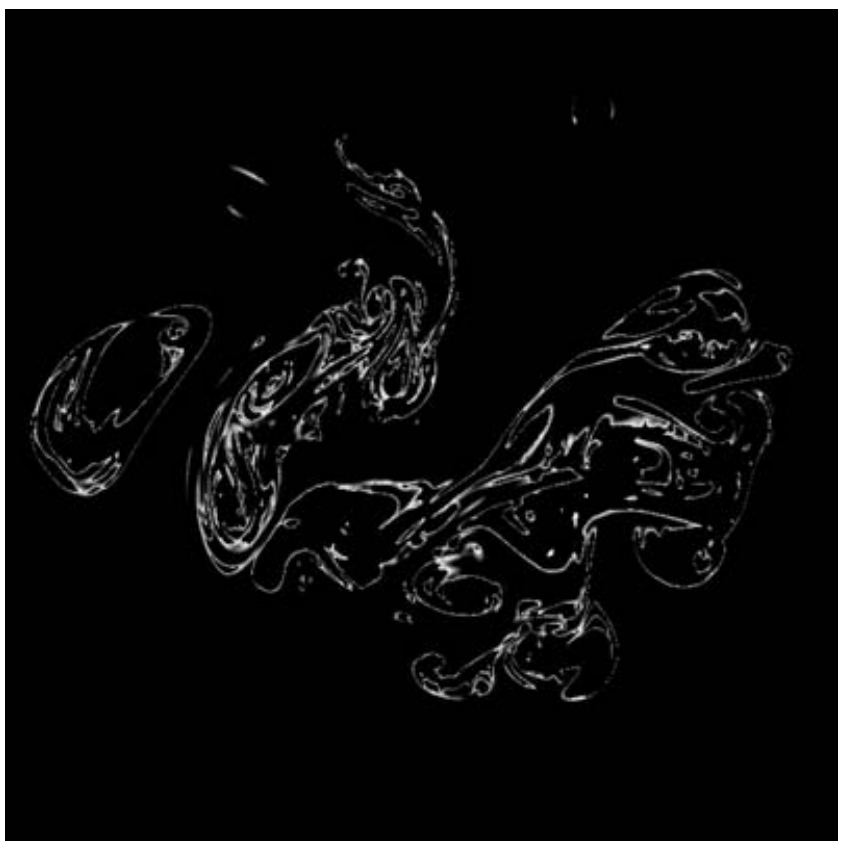

(b)

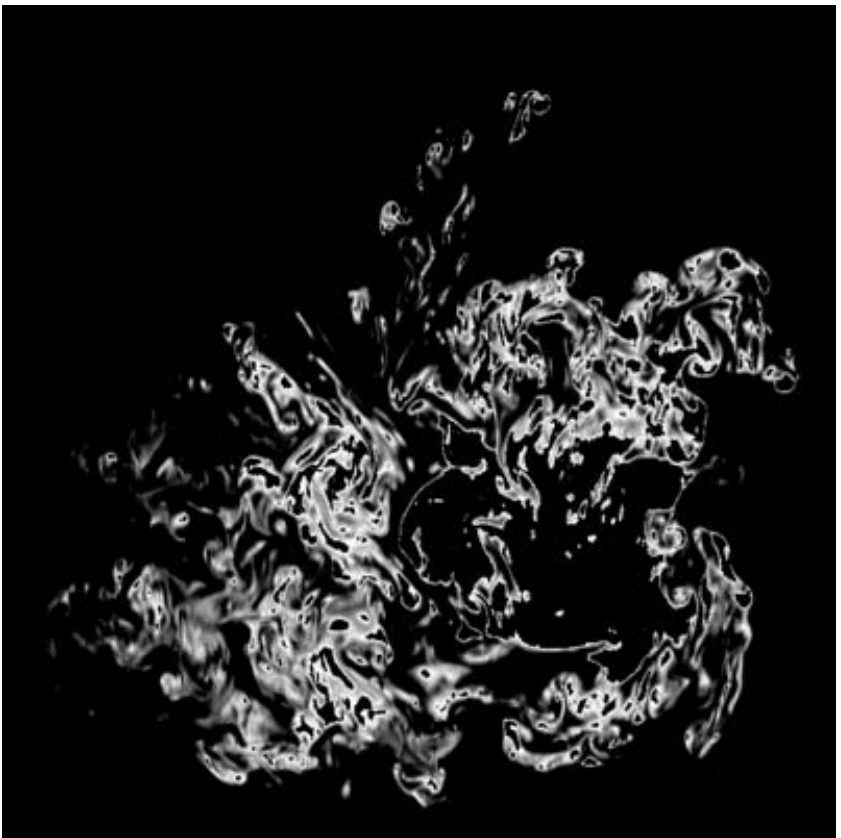

FIGURE 15. Concentrations in the range $0.025 \leqslant C \leqslant 0.045$ for $(a) R e_{j}=1.0 \times 10^{3}$ and (b) $R e_{j}=10 \times 10^{3}$ at $V_{r}=10$. Compare with figures 6 and 7 .

distribution. The most-probable concentration shifts to lower values with increasing Reynolds number, but the incremental change diminishes at the higher Reynolds numbers. Another concentration of interest is the mean mixed-fluid concentration, $C_{\text {mixed }}$, defined as the mean of all concentrations greater than a chosen value representing a cutoff between mixed and unmixed fluid. In this case, the cutoff 


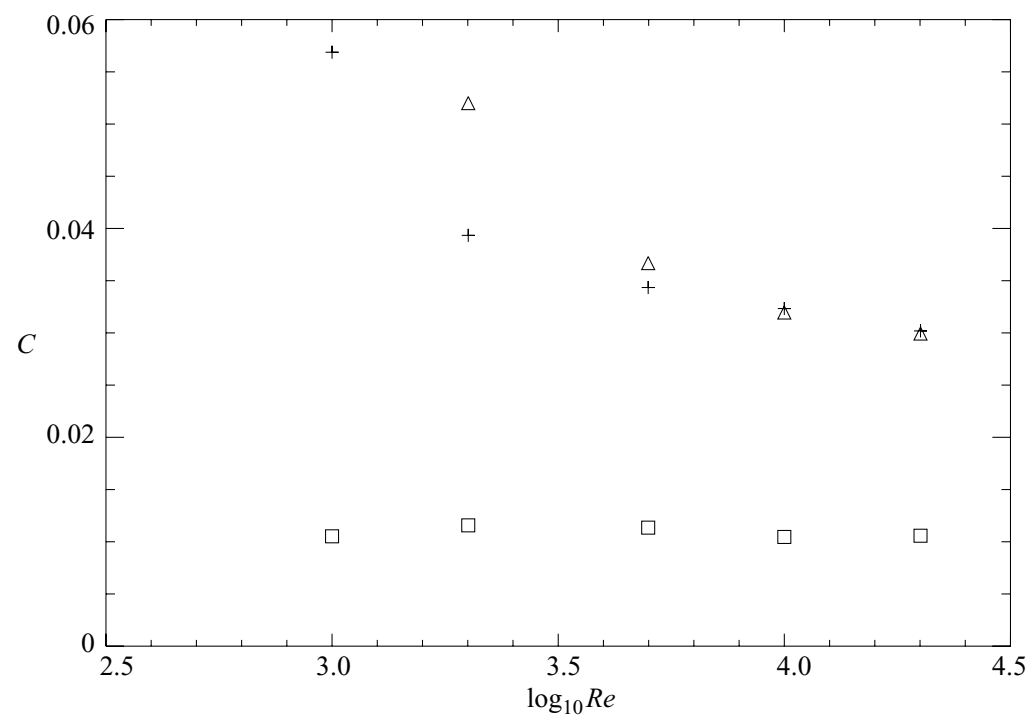

FIGURE 16. Peak, mixed-fluid, and mean concentrations versus Reynolds number for $V_{r}=10$. $\square$, mean concentration, $\bar{C} ;+$, mean mixed-fluid concentration, $C_{\text {mixed }}$, for $C>0.01 ; \triangle$, peak concentration, $C_{\text {peak }}$, defined as point of zero slope (for $R e_{j}=5.0,10$ and $20 \times 10^{3}$ ), or as point of maximum negative curvature (for $R e_{j}=2.0 \times 10^{3}$ ).

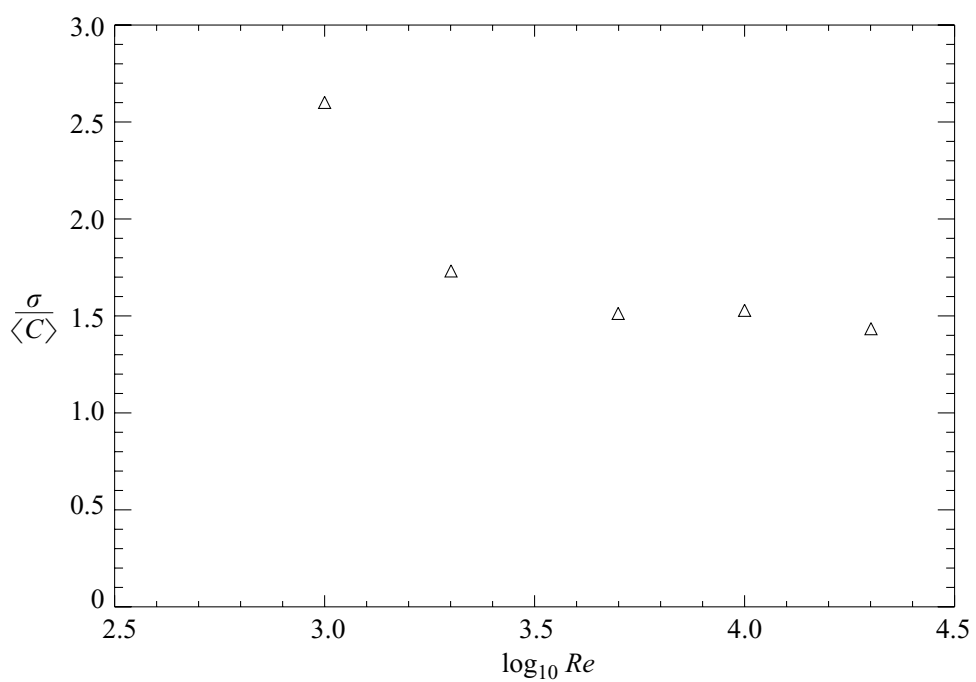

FIGURE 17. Normalized r.m.s. fluctuations of the scalar field versus Reynolds number for $V_{r}=10$.

concentration, $C=0.01$, was chosen to coincide with a local minimum that occurs in the PDF. The mean mixed-fluid concentration also asymptotes at the highest Reynolds numbers. Finally, the width of the PDF also declines and asymptotes with increasing Reynolds number. As seen in figure 17, the normalized standard deviation decreases rapidly before levelling off around $R e_{j}=10 \times 10^{4}$. The asymptotic levelling off of measures such as standard deviation and most-probable concentration for the transverse jet is consistent with a mixing transition that has been documented in other 


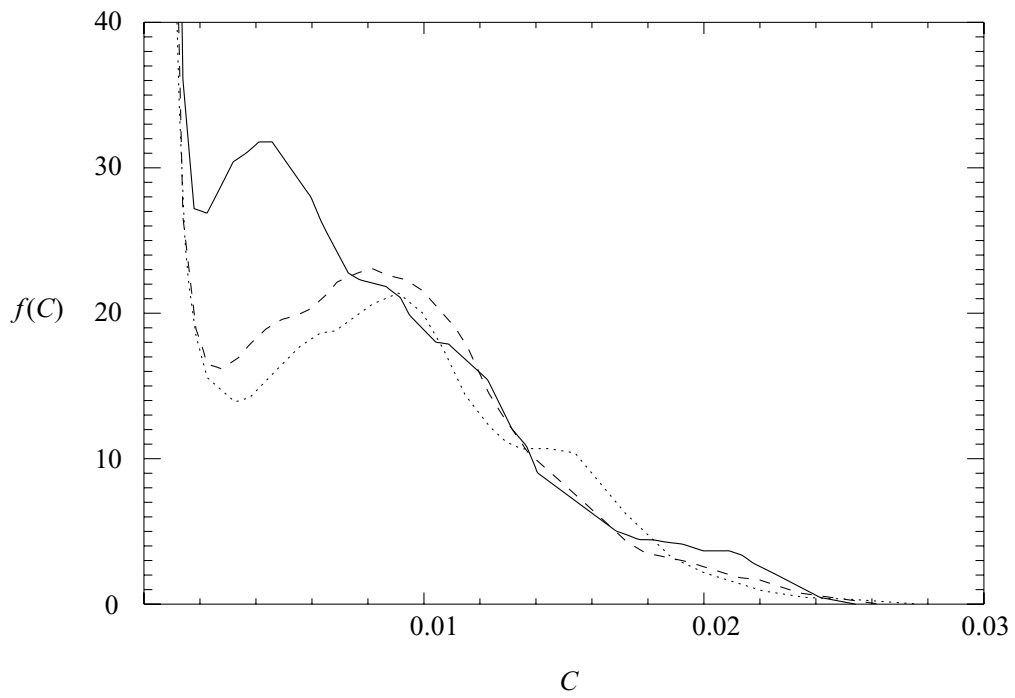

FIGURE 18. Jet-fluid concentration PDFs for jets discharging in a quiescent reservoir. Lines of increasing solidity denoting increasing $R e_{j}=4.5,9$ and $18 \times 10^{3}$ (Catrakis \& Dimotakis 1996, adapted from their figure 8).

flows to occur at comparable outer-scale Reynolds numbers of order $10^{4}$ (Dimotakis 2000).

It can be shown from control-volume analysis that the mean concentration in a transverse cross-section of the jet should remain constant for any Reynolds number, provided the velocity ratio is unchanged. The mean concentration, $\bar{C}$, depends linearly on velocity ratio,

$$
\bar{C}=\int_{-\infty}^{\infty} C f(C) \mathrm{d} C \simeq C_{0} V_{r} \frac{A_{j}}{A} .
$$

In the above expression, $A_{j}$ is the area of the nozzle exit, and $A$ is the area of a transverse plane whose boundary completely encloses the cross-section of the jet. When jet-plenum concentration, $C_{0}$, and velocity ratio, $V_{r}$, are fixed, as they are in the case of the present experiments, the mean concentration should be independent of the Reynolds number. This provides a test for the quantitative accuracy of the concentration measurements, and further verifies that the plenum concentrations and jet velocity ratios were well controlled for the test series. As shown in figure 16, the invariance of the mean concentration, $\bar{C}$, was verified for the present set of experiments.

\subsection{Entrainment and stirring compared with jets in quiescent reservoirs}

Differences in turbulent mixing can be seen between transverse jets and jets discharging into a quiescent reservoir. For comparison to the transverse-jet data (figure 14), figure 18 shows measured distributions of jet-fluid concentration for varying Reynolds number in the far-field $\left(x / d_{j}=275\right)$ of liquid-phase, quiescentreservoir jets. These jet measurements were made by Catrakis \& Dimotakis (1996) for a similar Reynolds-number range to the current transverse-jet experiments. At the lowest Reynolds number, $R e_{j}=4.5 \times 10^{3}$, the jet-fluid-concentration PDF of the quiescent-reservoir-jet displays a well-defined peak much like the PDF of the transverse jet at similar Reynolds numbers. However, as the Reynolds number 
increases, the height of the peak measured from the local minimum decreases, i.e. the relative height of the peak decreases. For jets without crossflow, the trend with increasing Reynolds number seems to be towards a smooth monotonic-decreasing distribution. We anticipate that the peak will disappear altogether with further increases in Reynolds number for the quiescent-reservoir jet. In contrast, for the transverse jet, the relative height of the distribution peak increases with Reynolds number. The trend in the transverse jet appears to be toward a strongly peaked distribution, as the scalar field is increasingly homogenized. The current data suggest that, for Reynolds numbers up to $R e_{j}=20 \times 10^{3}$, the trends in turbulent mixing with Reynolds number may be be different between transverse $\left(V_{r}=10\right)$ and quiescentreservoir jets. Additional comparisons at different downstream locations and velocity ratios would be required to confirm this hypothesis.

It is necessary to consider possible differences in entrainment between ordinary and transverse jets, because entrainment is a key step in what has been described as the three-stage process of turbulent mixing (e.g. Eckart 1948; Dimotakis 1986): entrainment; stirring; and molecular diffusion. (Entrainment is the engulfment of irrotational flow into the turbulent-flow region, stirring is the kinematic motion responsible for creating interfacial area between mixing species, and molecular mixing is due to diffusion.) The rate of entrainment of unmixed fluid, relative to the rate at which stirring and molecular diffusion act to homogenize the previously entrained fluid, helps to determine the distribution of the scalar field. This idea is illustrated in figure 19. The jet is modelled in a Lagrangian sense as a balloon being filled from a tap in similar fashion to the model developed for shear layers by Dimotakis \& Hall (1987). The balloon is initially filled with a fixed volume $V_{0}$ of $C=1$ fluid while the tap pours in $C=0$ fluid at varying volume flow rate $\dot{V}=e(t)$. For mixing jets, the original fluid in the balloon represents a parcel of plenum fluid that is injected into the crossflow, while the fluid that is poured in represents the fluid that is entrained into the jet in a convective frame moving with the jet fluid. The contents of the balloon are a Lagrangian parcel combining jet fluid and entrained crossflow fluid which cannot detrain, since entrainment and mixing can only proceed one way. Thus, the size of balloon (and the jet it represents) grows monotonically with time and downstream distance in an average sense. In the complete absence of stirring and molecular diffusion, the PDF of the contents of the balloon would be the sum of two delta functions, i.e. $f(C)=\left(1-C_{\text {mean }}\right) \delta(C)+C_{\text {mean }} \delta(C-1)$ (Dimotakis $\&$ Miller 1990). However, if stirring and diffusion act to mix the fluid, then intermediate, mixed concentrations would be generated within the balloon. In that case, the PDF would tend toward a delta function, $f(C)=\delta\left(C-C_{\text {mean }}\right)$, centred at the mean concentration, if stirring and molecular mixing rates are fast compared to the entrainment rate.

The mean concentration, $C_{\text {mean }}$, is determined by the ratio of jet fluid to entrained fluid, regardless of mixing rate, and is given by

$$
C_{\text {mean }}=\frac{V_{0}}{V_{0}+\int_{0}^{\infty} e(t) \mathrm{d} t}=\frac{1}{1+E / V_{0}},
$$

where $V_{0}$ is the original volume of $C=1$ fluid, $e(t)$ is the entrainment rate, and $E / V_{0}$ is the volume ratio of entrained to original fluid for that Lagrangian parcel of fluid.

The total mass flux of fluid through a plane perpendicular to the jet axis is

$$
\dot{m}=\int_{A_{j e t}} \rho \boldsymbol{u} \cdot \mathrm{d} \boldsymbol{A},
$$


(a)
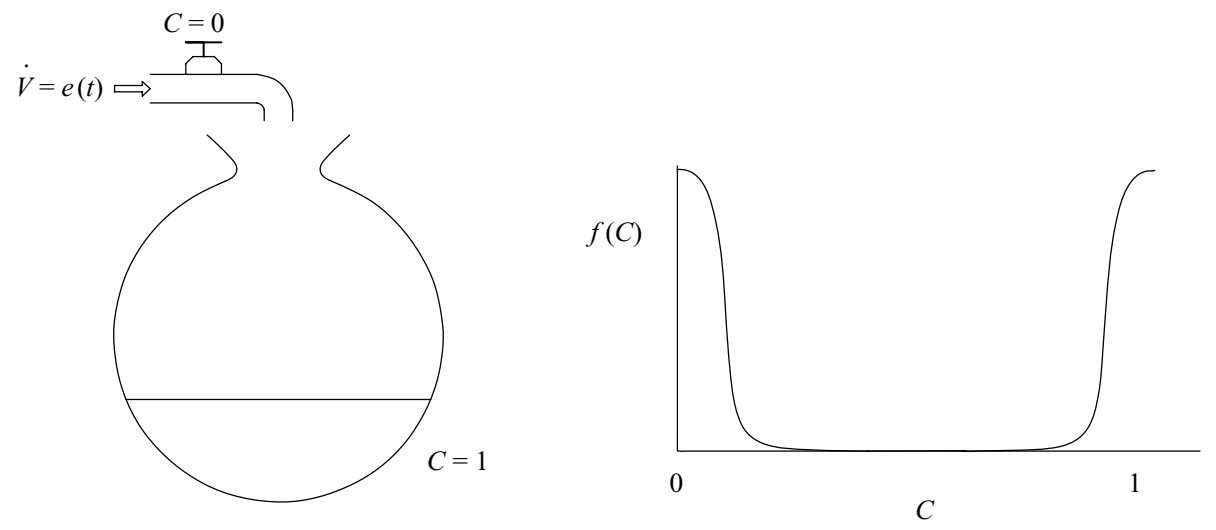

(b)
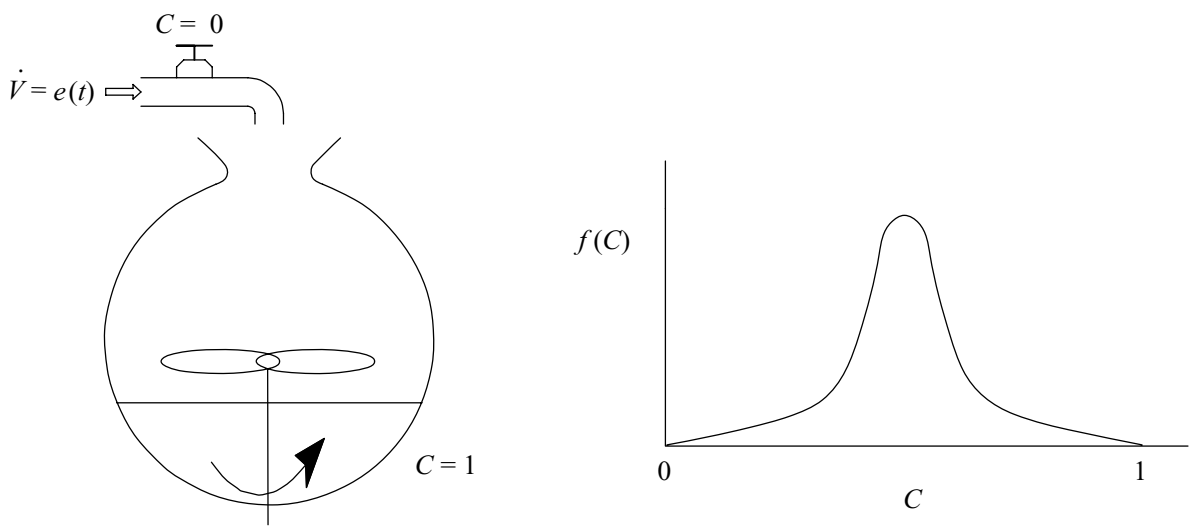

Stirrer

FIGURE 19. The jet-mixing process represented as a balloon being filled from a tap. The balloon represents a Lagrangian control-volume for a parcel of fluid initially comprised of pure jet fluid that entrains reservoir/crossflow fluid and mixes with it (a) Limited mixing absent stirring. (b) Mixing with enhanced stirring.

while the mass flux of dye is

$$
\dot{m}_{d y e}=\int_{A_{j e t}} C \rho \boldsymbol{u} \cdot \mathrm{d} \boldsymbol{A},
$$

where $\mathrm{d} \boldsymbol{A}$ has unit normal parallel to the jet axis. The mass flux of dye is conserved, and must be equal to the initial flux at the nozzle, i.e. $\dot{m}_{d y e}=\dot{m}_{0}$, since $C=1$ at the nozzle. In the far field of the jet, where $\boldsymbol{u} \simeq U_{\infty} \hat{\boldsymbol{x}}$, the ratio of mass fluxes is,

$$
\frac{\dot{m}_{\text {dye }}}{\dot{m}} \simeq \frac{\int_{A_{j e t}} C \mathrm{~d} A}{\int_{A_{j e t}} \mathrm{~d} A}=\frac{\int_{A_{j e t}} C \mathrm{~d} A}{A_{\text {jet }}} .
$$

This ratio of dye flux to total flux is simply the mean flux, $C_{\text {mean }}$, which can be measured from the image data. Thus, the entrainment of transverse jets can be deduced from measurements of concentration and compared to expressions proposed by previous investigators. 
It should be noted that the mean concentration, $C_{\text {mean }}$, discussed above is the average concentration of a Lagrangian parcel of jet fluid mixed with entrained fluid. It differs subtly from the $\bar{C}$ that was previously defined in (17) as the measured mean concentration over a field of view containing a cross-section of the jet as well as surrounding tunnel fluid. Because the field-of-view of the measurement contains additional (un-entrained) surrounding fluid in addition to the mixed jet fluid, $C_{\text {mean }} \geqslant \bar{C}$. (The equality would hold only if concentration is measured for an area containing only the jet fluid and entrained tunnel fluid, i.e. excluding the surrounding tunnel fluid.) On the other hand, the mean concentration, $C_{\text {mean }}$, is less than the mixed-fluid concentration, $C_{\text {mixed }}$, which was previously defined as the average over the measurement plane of all concentrations greater than $C=0.01$ (figure 16). The mean concentration is thus constrained to be,

$$
\bar{C} \leqslant C_{\text {mean }}<C_{\text {mixed }},
$$

Based on measurements at $x / d_{j}=50$ for the transverse jet, $C_{\text {mixed }}=0.03$ and $\bar{C}=0.01$ (see figure 16). Using (18) and the measured $C_{\text {mixed }}$ and $\bar{C}$ for the $V_{r}=10$ jet, the ratio of entrained fluid to jet fluid at $x / d_{j}=50$ is found to be within the limits:

$$
32<\frac{E}{V_{0}} \leqslant 99 .
$$

For comparison, Hasselbrink \& Mungal (2001) and Hanm, Orozco \& Mungal (2000) estimated the entrainment rate of transverse jets by considering the momentum exchange between crossflow and jet fluid necessary to turn the jet to an experimentally determined trajectory. They proposed an expression for total mass flux through a section perpendicular to the jet axis in the transverse jet as:

$$
\frac{\dot{m}}{\dot{m}_{0}}=1+\frac{V_{r}}{A B}\left(\frac{x}{V_{r} d_{j}}\right)^{1-B},
$$

where $A \simeq 2.1$ and $B \simeq 0.29$ are experimentally determined constants. This expression is valid in the far field, for $s / V_{r}^{2} d_{j} \geqslant 0.3$ by the definition of Smith \& Mungal (1998), where $s$ is the arclength from the jet exit. Substituting (24) into (21) then yields:

$$
C_{\text {mean }} \simeq \frac{1}{1+\frac{V_{r}}{A B}\left(\frac{x}{V_{r} d_{j}}\right)^{1-B} .}
$$

This predicts a mean concentration $C_{\text {mean }}\left(x / d_{j}=50\right) \simeq 0.02$ and entrained-to-crossflow fluid ratio $E / V_{0} \simeq 51$ for the $V_{r}=10$ jet, which is consistent with the range of values inferred from the concentration measurements (equations (22) and (23)).

For a turbulent, axisymmetric jet in a quiescent reservoir, the total mass flow rate was found by Ricou \& Spalding (1960) to be:

$$
\frac{\dot{m}}{\dot{m}_{0}}=1+0.32 \frac{s}{d_{j}} .
$$

This is plotted in figure 20 along with the normalized mass flux versus arclength, $s$, from the jet exit for the $V_{r}=10$ transverse jet. In the figure, the lines transition from dashed to solid lines at the beginning of the far field for transverse and regular jets, as defined by Smith \& Mungal (1998) and Dahm \& Dimotakis (1990), respectively. At the downstream location of the present experiments, $x / d_{j}=50$ (equivalent to $\left.s / d_{j}=67\right)$, the $V_{r}=10$ transverse jet has 2.3 times the total mass-flow rate of an 


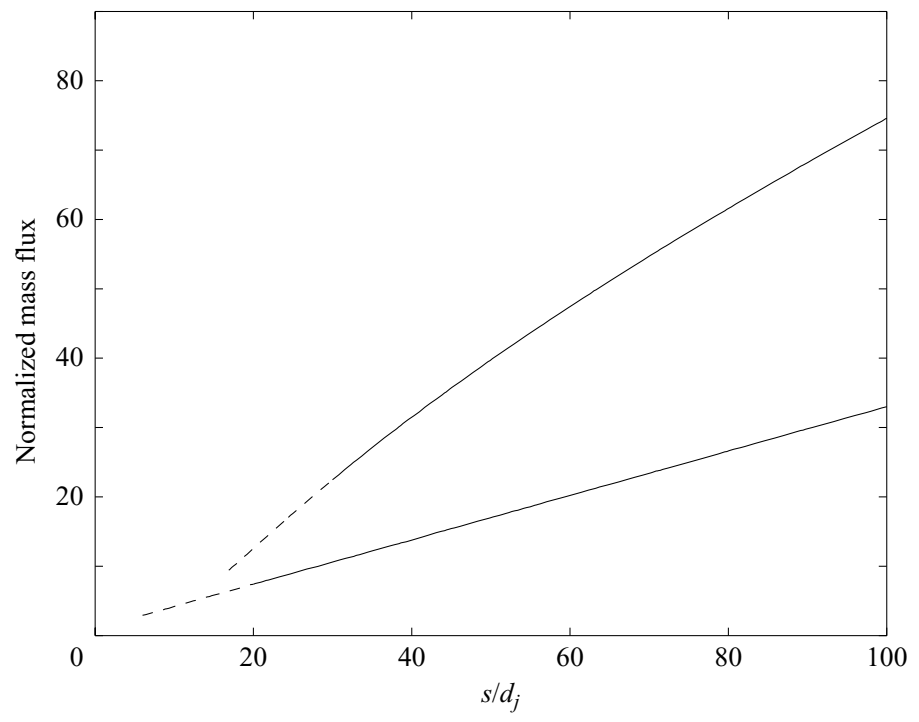

FIGURE 20. Normalized total mass flux, $\dot{m} / \dot{m}_{0}$, versus arclength from jet exit. Upper line: transverse jet for $V_{r}=10$. Lower line: jet in quiescent reservoir.

ordinary jet of the same initial mass-flow rate. If extrapolated further, the two lines will eventually cross in the extreme far field, as $\dot{m} / \dot{m}_{0} \propto\left(s / d_{j}\right)^{1-B}$, where $B \simeq 0.29$ for the transverse jet, while $\dot{m} / \dot{m}_{0} \propto s / d_{j}$ for the ordinary jet. However, for the $V_{r}=10$ transverse jet, the estimated cross-over in mass flux occurs at $s / d_{j} \simeq 2800$. Thus, for most practical purposes, the high-velocity-ratio transverse jet has entrained more fluid than a jet of the same initial mass flux discharging in a quiescent reservoir.

As discussed in the previous section, the growing peaks in the PDF of jetfluid concentration indicate that the scalar field of the transverse jet is increasingly homogenized with increasing Reynolds numbers. This implies that stirring, relative to entrainment rate, is enhanced at higher Reynolds numbers. Yet, in comparison to the jet discharging in a quiescent reservoir, the $V_{r}=10$ transverse jet has a greater entrainment rate and total mass flux at $x / d_{j}=50$. The spatial homogenization of the scalar field in the far field occurs in spite of the transverse jet's relatively high entrainment rate. The high-velocity ratio, turbulent transverse jet is an efficient mixer in the sense that it rapidly entrains unmixed free-stream fluid, and homogenizes the jet-fluid-concentration field.

\section{Scalar increment statistics}

While the statistics of concentration fields in transverse jets can be described with scalar PDFs $(\S 3)$, the structure of the scalar field can be assessed in terms of the distribution of spatial differences, $f\left(\Delta_{r} C\right)$. As introduced in (1), the probability distribution of scalar differences, also known as scalar increments, gives the probability of finding a concentration $C+\Delta C$ at a vector distance $r$ away from a point of concentration $C$. The statistics of scalar differences are connected to the structure functions for a turbulent scalar field and have implications for the KolmogorovOboukhov-Corrsin (KOC) theory of turbulent mixing of passive scalars (Oboukhov 1949; Corrsin 1951; an overview of passive-scalar turbulence is given by Shraiman \& 

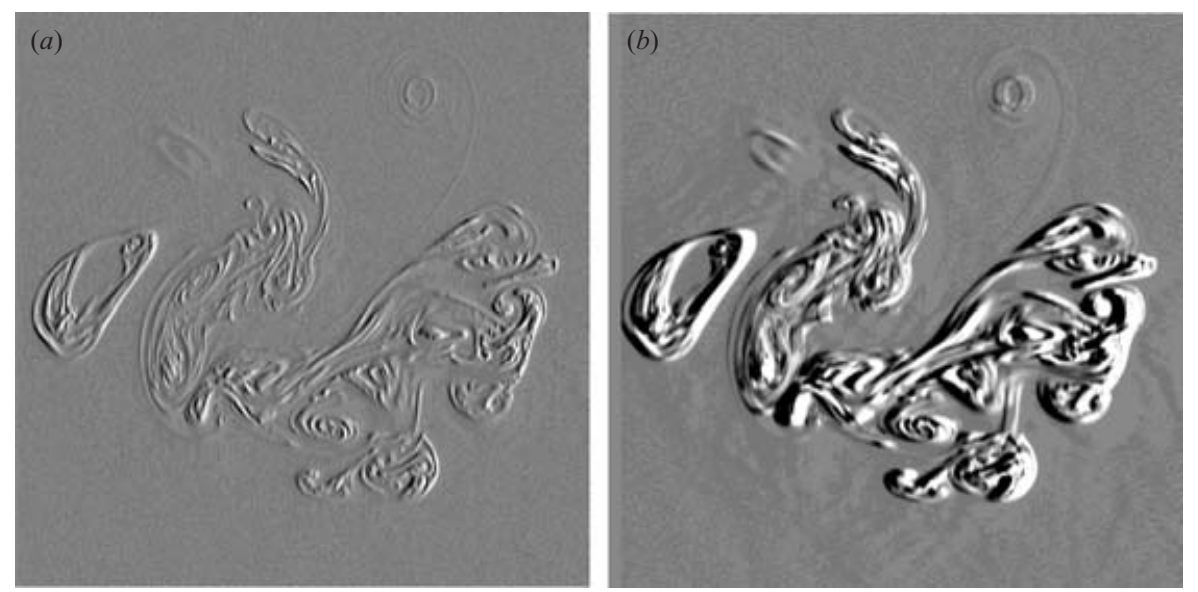

FIGURE 21. Scalar-difference field for $R e_{j}=1.0 \times 10^{3}, V_{r}=10$ jet. (a) Horizontal shift of $0.3 \times 10^{-2} \delta_{h}$. (b) Horizontal shift of $2.1 \times 10^{-2} \delta_{h} . \Delta_{r} C=0$ is shown as grey, and negative and positive values are shown as black and white, respectively.

Siggia 2000). In this paper, the distribution of scalar differences is investigated primarily for the evidence they provide for intermittency and anisotropy in the scalar field.

Scalar increments are typically studied with point measurements of the scalar field, for example, with temperature probes (Antonia et al.1984; Ould-Rouis et al. 1995; Mydlarski \& Warhaft 1998). However, two-dimensional image data of the type collected in the present experiments allow novel whole-field measurement of scalar increments of the concentration field (figure 21). Normalized and calibrated images measuring scalar concentration fields are spatially displaced by a vector distance $\boldsymbol{r}$ and subtracted from themselves. For instance, figure 21 shows twodimensional scalar-difference fields for small and large horizontal displacements, $\boldsymbol{r} / \delta_{h}=0.3 \times 10^{-2} \widehat{z}$ and $\boldsymbol{r} / \delta_{h}=2.1 \times 10^{-2} \widehat{z}$, respectively. The probability distribution of scalar increments is then computed as the normalized histogram of the scalardifference field. To prevent the PDF from being dominated by differences between the completely unmixed crossflow fluid and the jet, the distribution of scalar differences is conditioned on both measurement 'points' being within the instantaneous body of the jet. This is accomplished by making the distribution conditional on the intersection between shifted and unshifted jet body, defined from the image data by a minimum scalar threshold. The procedure is conceptually equivalent to requiring that the two measurement 'probes' lie within the jet body. (The condition is necessary because of the inhomogeneity and spatial confinement of the image data. Without it, the distribution of scalar difference would be dominated at large separation distances by differences between points within the jet and points outside of the jet. In that case, the distribution of scalar increments for a spatially confined scalar field would simply tend toward the concentration PDF, $f(C)$ and its reflection, $f(-C)$ for large $r$.)

\subsection{Intermittency and the distribution of scalar increments}

Distributions of conditional scalar increments at two Reynolds numbers, $\operatorname{Re}_{j}=2.0 \times$ $10^{3}$ and $R e_{j}=20 \times 10^{3}$, are shown in figure 22. The distributions were computed 

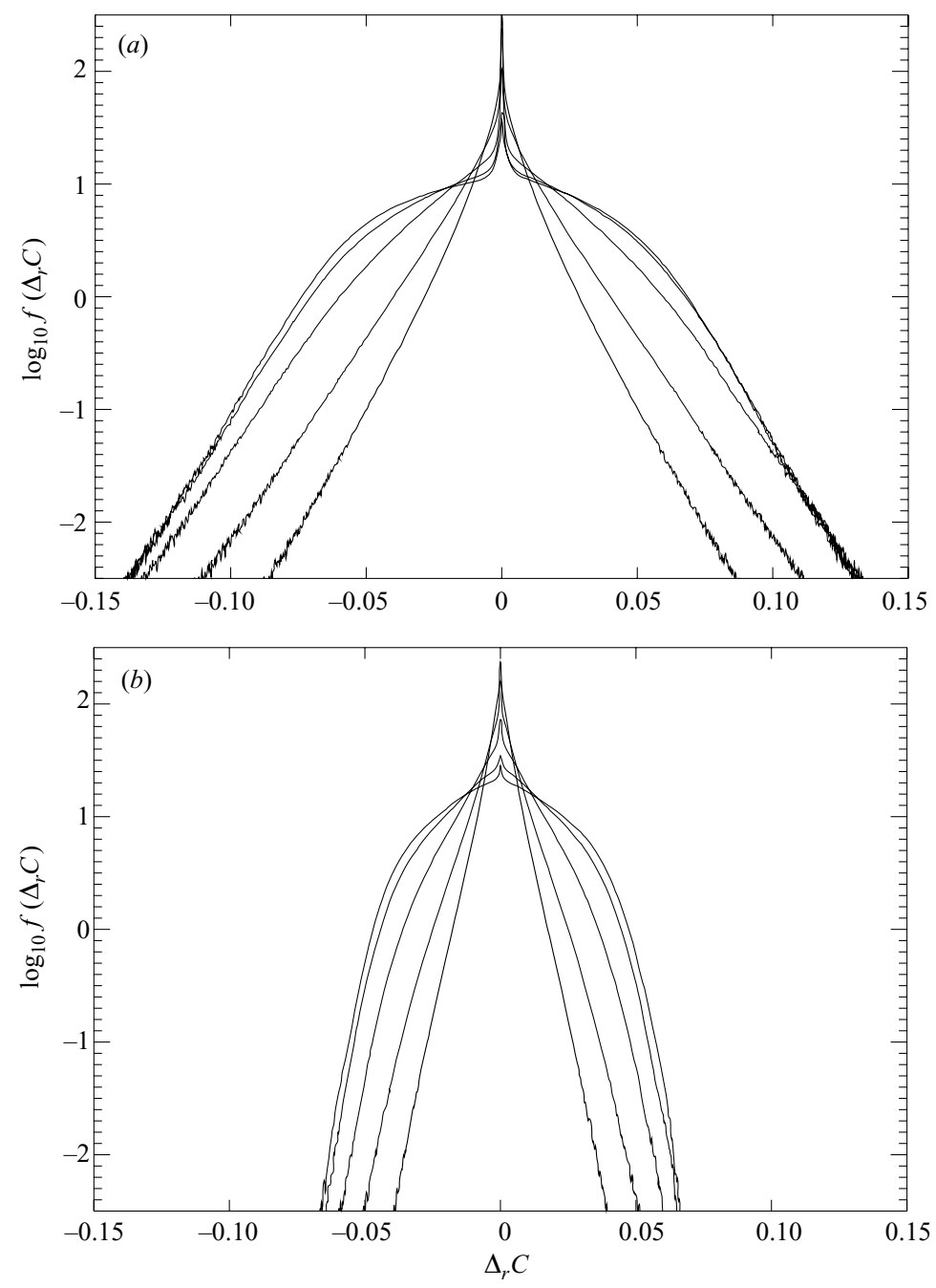

FiguRE 22. Scalar-increment PDFs at $V_{r}=10$ for two different Reynolds numbers and varying horizontal separation distances, $r$. Innermost, triangular PDFs are for smallest separation distances and outermost, broad-shouldered PDFs are for largest separations. (a) $R e_{j}=2.0 \times 10^{3}$ and separation distances $\boldsymbol{r} / \delta_{h}=0.2,0.4,1.7,7.0$, and $14 \times 10^{-2} \widehat{z}$. (b) $R e_{j}=20 \times 10^{3}$ and separation distances $\boldsymbol{r} / \delta_{h}=0.3,0.5,2.0,8.1$ and $16 \times 10^{-2} \widehat{z}$.

for varying horizontal separation distances in the range $0.2 \times 10^{-2} \leqslant|\boldsymbol{r}| / \delta_{h} \leqslant 14 \times$ $10^{-2}$, where $\delta_{h}$ was previously defined $(\S 3)$ to be the horizontal size of the jet based on mean concentration. As seen from the figure, the PDF becomes narrower with increasing Reynolds number at all separation distances, i.e. the probability for large differences in the scalar field decreases with increasing Reynolds number. Large differences in mixed-fluid concentration between two spatially separated points become rarer as the jet fluid is increasingly homogenized at high Reynolds numbers. This increased spatial homogenization of the scalar field was also seen in the growing preferred-concentration peaks in the PDF of jet-fluid concentration (figure 14), and the decreasing variance of the scalar field (figure 17). 

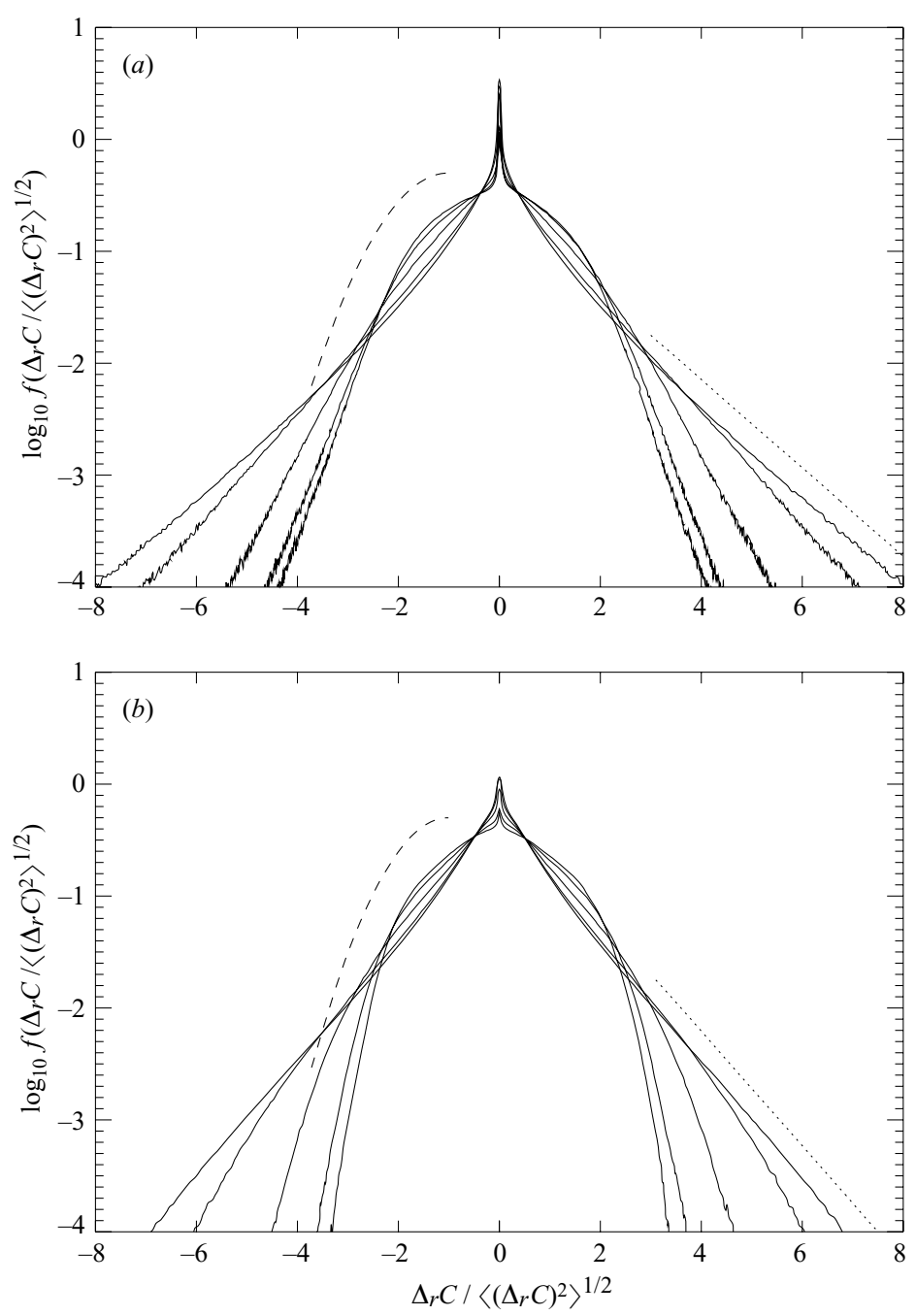

FIGURE 23. Standard-deviation-normalized PDFs of scalar increments at $V_{r}=10$ for two Reynolds numbers and varying horizontal separation distances, $r$. Gaussian (dashed) and exponential (dotted) distributions shown for comparison. Exponential-tailed triangular PDFs are for smallest separation distances and Gaussian-shouldered innermost PDFs are for largest separations. (a) $R e_{j}=2.0 \times 10^{3}$ and separation distances $r / \delta_{h}=0.2,0.4,1.7,7.0$ and $14 \times$ $10^{-2} \widehat{z}$. (b) $R e_{j}=20 \times 10^{3}$ and separation distances $\boldsymbol{r} / \delta_{h}=0.3,0.5,2.0,8.1$ and $16 \times 10^{-2} \widehat{z}$.

As the separation distance changes for a given Reynolds number, the shape of the scalar increment PDF evolves from a broad-shouldered distribution to a longtailed distribution. Figure 23 shows scalar increment PDFs normalized by their own standard deviation. The distributions are plotted for varying horizontal separation distances, $r$. A growing 'spike' is seen at $\Delta_{r} C \simeq 0$ as the separation distance decreases. At the largest distances, the PDF has narrow tails and shoulders which are Gaussian in shape. For smaller separation distances, the PDF is long-tailed and has an exponential or stretched-exponential form. For comparison, exponential and Gaussian distributions are plotted in the figure. The observed exponential or 
stretched-exponential behaviour of the distributions is consistent with previous results for scalar temperature increments in Rayleigh-Bénard convection (Ching 1991), grid turbulence (Mydlarski \& Warhaft 1998), boundary layers (Ould-Rouis et al. 1995), and plane jets (Antonia et al. 1984). Using novel whole-field measurements of scalar increments, the present experiments extend the list of turbulent flows for which long-tailed distributions of scalar increments are found to liquid-phase transverse jets. (In making comparisons, it should be noted that the Schmidt number of the present experiment is two orders of magnitude higher than the Prandtl number of the previous temperature measurements.)

The long tails and peak at the origin of the scalar-increment distribution are believed to arise from plateau-cliff structures in the scalar field. Such structures, consisting of integral-scale-sized 'plateaux' of nearly homogeneously mixed fluid separated by intense gradient sheets, or 'cliffs', have been documented in a variety of turbulent mixing flows (discussed below), and are also seen in the scalar field of the transverse jet. Figure 24 shows the jet-fluid concentration in a transect of the transverse jet at $R e_{j}=10 \times 10^{3}$. In this transect, the concentration is arranged in three bands (plateaux), separated by large jumps (cliffs) in concentration. Relatively small fluctuations in concentration occur within the bands. Such an intermittent scalar field can produce a spiked long-tailed distribution of scalar increment in the following manner. Plateaux of nearly constant scalar concentration in the transverse jet contribute to the large small-concentration-difference peak at the origin of the distribution of $\Delta_{r} C$, while cliffs separating the concentration plateaux produce large fluctuations which contribute to the long tails of the scalar-increment distribution. The spiked long-tailed distribution of scalar increments is thus evidence that the scalar field is intermittent and contains plateaux and cliffs which persist for Reynolds number up to $R e_{j} \leqslant 20 \times 10^{3}$.

Large-scale plateaux of well-mixed fluid, separated by cliffs of sharp concentration gradients, appear to be characteristic of a wide variety of turbulent mixing flows. Such scalar plateaux and cliffs in physical space (or, equivalently, ramp-cliffs for timeseries measurements) have previously been observed in experiments on mixing in turbulent shear flows such as wakes (Gibson, Friehe \& McConnell 1977), boundary layers (Mestayer et al. 1976; Gibson et al.1977), and jets (Uberoi \& Singh 1975; Sreenivasan, Antonia \& Britz 1979; Antonia et al.1986; Dahm \& Dimotakis 1990; Yoda et al.1992). Such ramp-cliff structures have also been found in grid turbulence with a superimposed mean-temperature profile (Budweig, Tavoularis \& Corrsin 1985; Thoroddsen \& Van Atta 1992; Tong \& Warhaft 1994), despite the absence of mean shear, entrainment and large-scale anisotropy of the velocity field. Numerical simulations in two- (Holzer \& Siggia 1994) and three- (Pumir 1994) dimensions show the same basic structure of the scalar field in physical space: relatively wellmixed regions separated by cliffs where the scalar gradient is very large. As noted by Warhaft (2000), it is remarkable that ramp-cliff structures are observed in the two-dimensional simulations of Holzer \& Siggia, which assumed a Gaussian random velocity field rather than a velocity field that is a solution of the Navier-Stokes equations. Experiments (Antonia et al. 1986) and simulations (Pumir 1994) indicate that cliffs tend to develop where the flow is hyperbolic (at a diverging separatrix), while elliptic regions are better mixed. Based on experiments in heated grid turbulence, Tong \& Warhaft (1994) concluded that the cliffs persist and become even sharper and more intense with increasing Reynolds number up to at least $R e_{\lambda}=130$. In doing so, the cliff-plateau structures contribute deeper and deeper into the tails of the scalar-derivative PDF with increasing Reynolds number. (The scalar-increment 
(a)

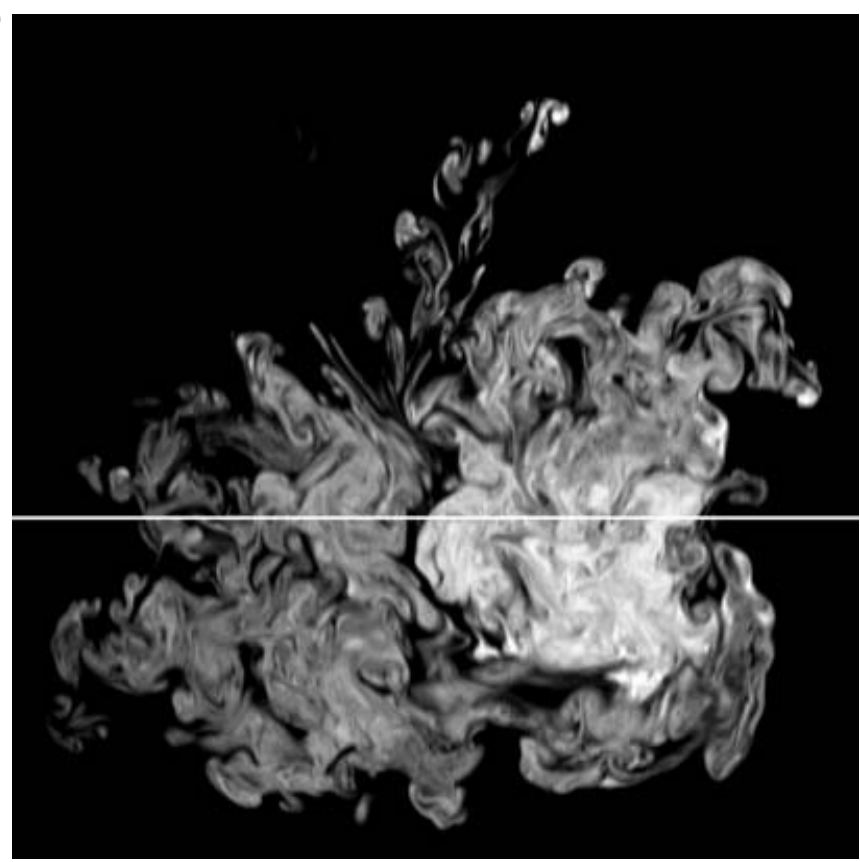

(b)

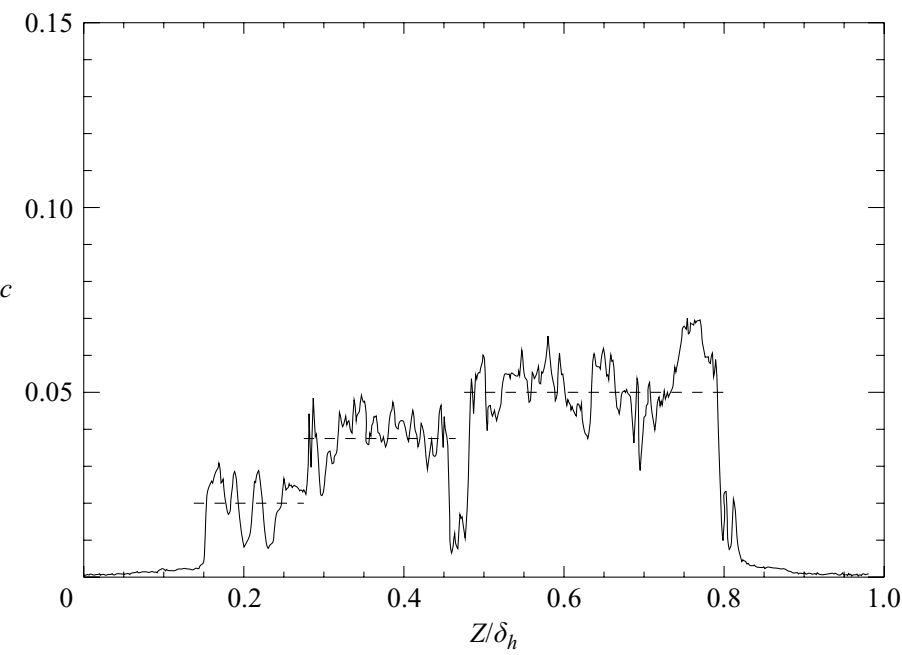

FIGURE 24. Concentration in a transect of the jet at $R e_{j}=10 \times 10^{3}$ and $V_{r}=10$.

(a) Location of transect. (b) Concentration versus position.

PDF would approach the scalar-derivative PDF in the limit of $\boldsymbol{r} \rightarrow 0$.) The basic plateau-cliff or ramp-cliff structure of the scalar field has also been associated with small-scale anisotropy, an issue that is discussed in the following sections.

\subsection{Anisotropy and scalar increments in differing directions}

The measured distributions of scalar increments suggest that the large scales (plateaux) of the scalar field are separated by small scales (cliffs). In a sense, this is a coupling of 
the large and small scales, which introduces the possibility that the scalar field may be anisotropic even at small length scales (e.g. reviews by Sreenivasan \& Antonia 1997; Shraiman \& Siggia 2000; Warhaft 2000). There has long been evidence of a lack of scalar isotropy in turbulent mixing flows at both inertial and dissipation scales. A persistent (even at high Reynolds numbers) non-zero skewness of the scalar derivative has been reported in shear flows (Mestayer et al.1976; Gibson et al.1977; Sreenivasan et al. 1979) as well as isotropic grid turbulence with a linear temperature profile (Tavoularis \& Corrsin 1981; Budwig et al. 1985; Tong \& Warhaft 1994). The skewness of the scalar derivative is a consequence of the ramp-cliff structures in the scalar field, which can be preferentially aligned in a turbulent flow. In shear flows, the converging-diverging separatrices that form the cliffs are inclined along the principal-strain direction on average (Antonia et al.1986). In flows without mean shear, the separatrices are randomly aligned; however, those that happen to be aligned with the mean scalar gradient will tend to create ramp-cliff structures (Holzer \& Siggia 1994; Warhaft 2000). In either case, the cliffs in the scalar field are preferentially oriented and sharp, thus resulting in scalar anisotropy at both large and small scales. In addition to the reported non-zero skewness of the scalar field, experiments have investigated the morphology of turbulent scalar fields with three-point correlations, $\left\langle C\left(\boldsymbol{x}_{A}\right) C\left(\boldsymbol{x}_{B}\right) C\left(\boldsymbol{x}_{C}\right)\right\rangle$, where the net result depends on the distribution of cliff orientations and the geometry of the triangle formed by the three measurement points. Experiments on heated grid turbulence found scalar anisotropy using such measures at both the dissipation and inertial scales of the scalar field (Mydlarski \& Warhaft 1998). In similar spirit to the three-point correlations of the scalar field, the probability distribution of differences between two points in the scalar field can be measured for separations in different directions to investigate possible anisotropy and structure at different length scales in the scalar field.

Figure 25 shows distributions of scalar increments for separations in two orthogonal directions at $R e_{j}=2 \times 10^{3}$. At the larger separation distance $\left(1.7 \times 10^{-2} \delta_{h}\right)$, the PDFs are nearly identical for horizontal and vertical separations of the same distance. However, at a smaller separation distance $\left(0.2 \times 10^{-2} \delta_{h}\right)$, the distributions are markedly different. The PDF is narrower for vertical increments than horizontal increments, indicating the scalar field has less variation in the vertical than in the horizontal direction. Increments in different directions for $R e_{j}=20 \times 10^{3}$ also show anisotropy of the scalar field (figure 26). Thus, the difference in the distributions of scalar increments for separations in two directions suggests that the scalar field has small length-scale, vertically aligned ( $y$-axis) structure for the Reynolds-number range investigated.

More insight into the spatial localization and possible sources of the small-scale anisotropy of the scalar field can be gained by directly visualizing the scalar difference field $\Delta C$ for small separations in two directions. Figure 27 compares scalar-difference fields for small horizontal and vertical displacements of $0.2 \times 10^{-2} \delta_{h}$. The same linear intensity scaling is used to display concentration values in both images. Higher contrast in the images indicates larger differences in concentration, which contribute to the tails of $f\left(\Delta_{r} C\right)$. Visual examination of the scalar-increment fields reveals that large scalar differences (high contrast, nearly white or black features in the images) are more prominent and common for horizontal separations than vertical separations of the same distance. This difference in contrast is visual evidence of anisotropy, indicating that there is a preferred vertical orientation for the small scales of the scalar field. The small-scale scalar anisotropy appears strongest near the centreline of the jet (between the counter-rotating cores), where wispy 'fingers' extend up into the 

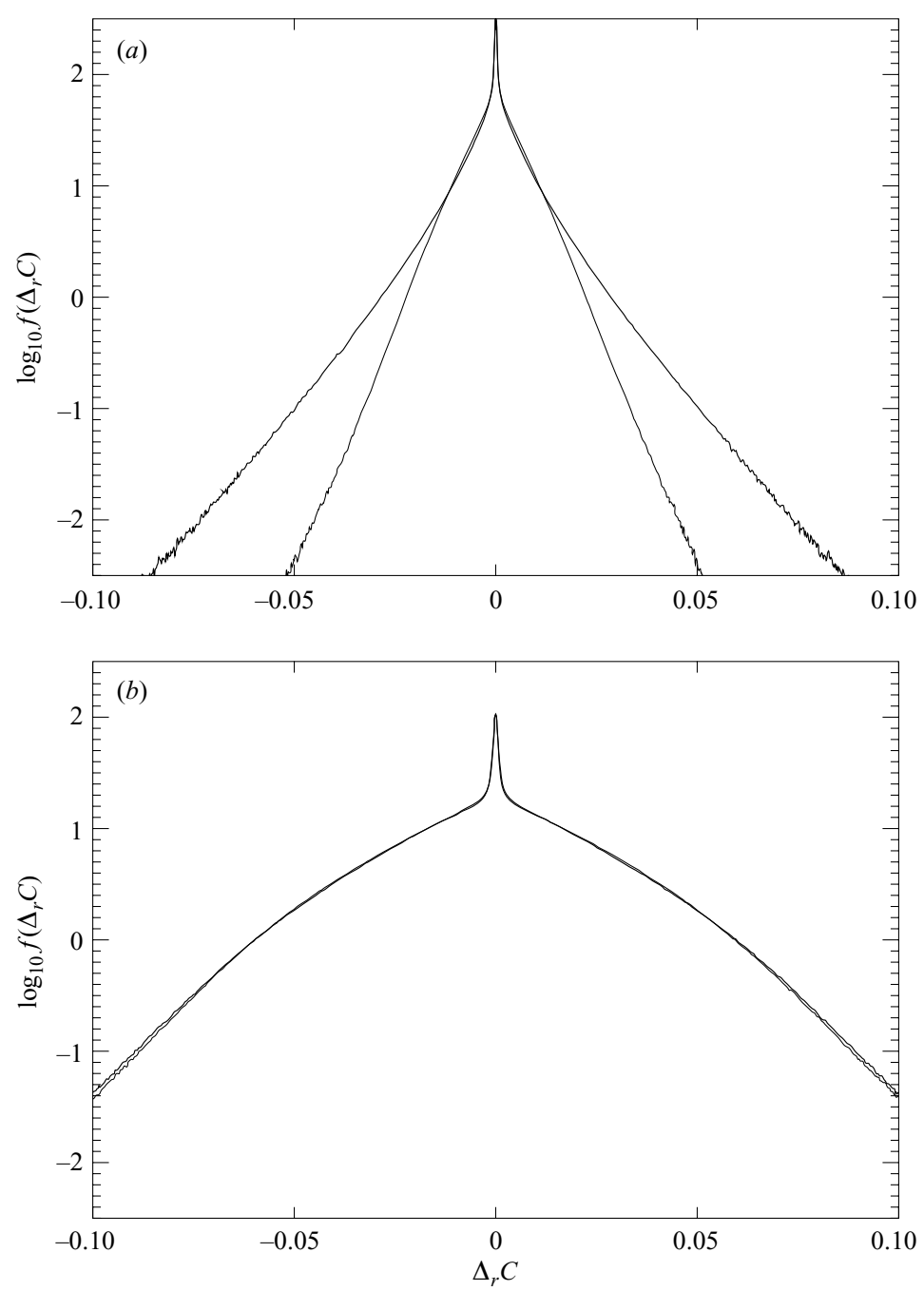

FIGURE 25. PDFs of scalar increments at $V_{r}=10$ for the same horizontal and vertical separations. $R e_{j}=2.0 \times 10^{3}$ and $x / d_{j}=50$. (a) Outer PDF is for horizontal separations of $\boldsymbol{r} / \delta_{h}=2.2 \times 10^{-3} \widehat{z}$, and inner PDF is for vertical separations of $\boldsymbol{r} / \delta_{h}=2.2 \times 10^{-3} \widehat{\boldsymbol{y}} .(\mathrm{b}) \boldsymbol{r} / \delta_{h}=$ $17 \times 10^{-3} \widehat{z}$ and $17 \times 10^{-3} \widehat{y}$.

wake. In the following section, additional evidence is presented for local anisotropy of the scalar field of the transverse jet, and a possible mechanism for the anisotropy is presented.

\section{Anisotropy of scalar field}

The anisotropy of the scalar field that was seen in the distribution of scalar increments can also be seen in measures such as one- and two-dimensional power spectra. One-dimensional power spectra can be computed from image data of the concentration field by taking transects of the scalar field. The spectra are computed for each row or column of the image and then ensemble-averaged over the entire 

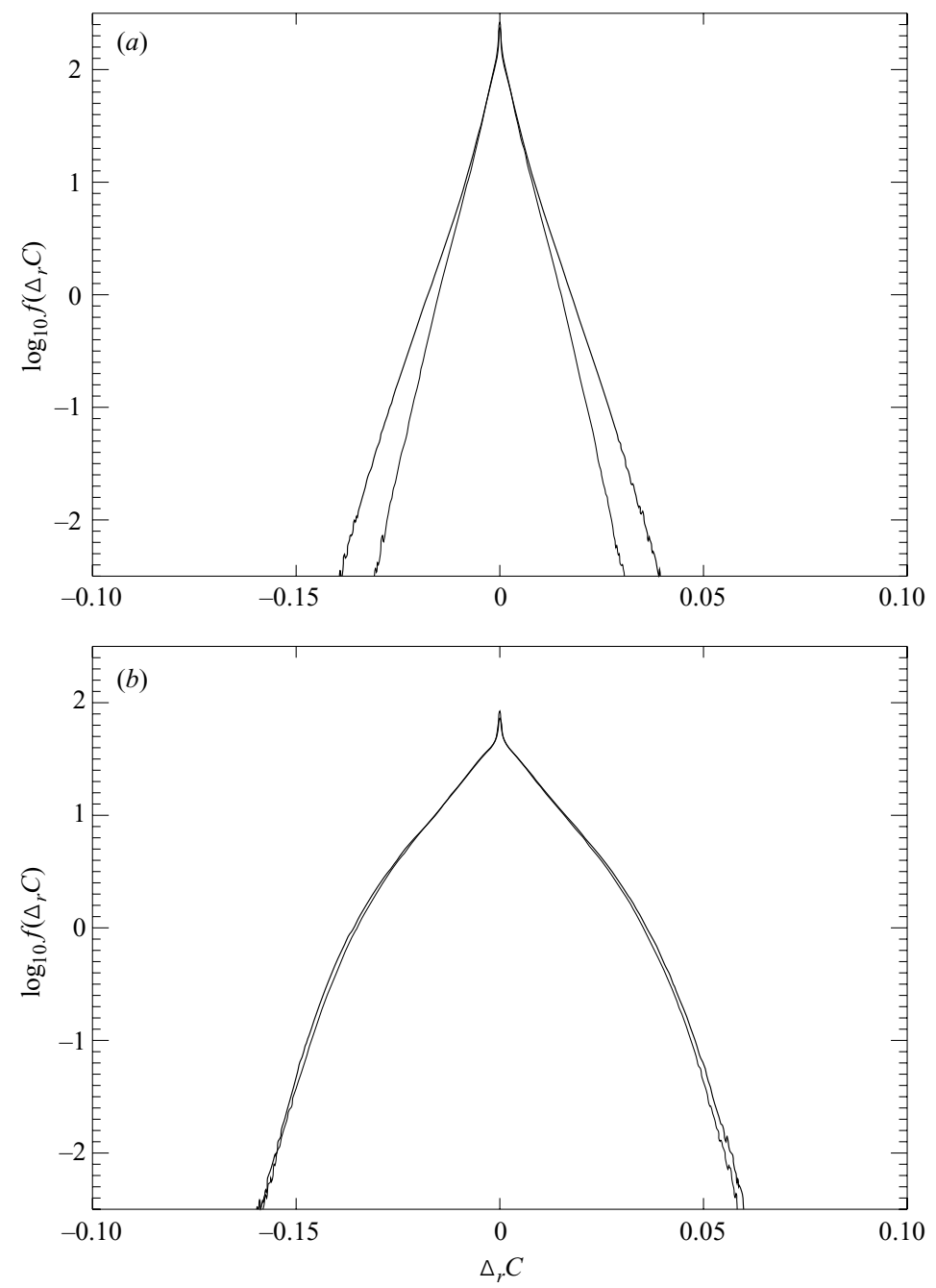

FiguRE 26. As figure 25, but for $R e_{j}=20 \times 10^{3}$. (a) Outer PDF is for horizontal separations of $\boldsymbol{r} / \delta_{h}=2.5 \times 10^{-3} \widehat{z}$, and inner PDF is for vertical separations of $\boldsymbol{r} / \delta_{h}=2.5 \times 10^{-3} \widehat{\boldsymbol{y}}$ pixels. (b) $\boldsymbol{r} / \delta_{h}=20 \times 10^{-3} \widehat{z}$ and $20 \times 10^{-3} \widehat{y}$.

image and averaged again over the sequence of 508 images. Hann-windowing is used to prevent aliasing. The scalar power spectra are normalized by the scalar variance and non-dimensionalized by the mean width of the jet, $\delta_{h}$, i.e.

$$
\hat{S}_{C} \equiv \frac{S_{C}\left(k \delta_{h}\right)}{\delta_{\mathrm{h}}\left\langle C^{\prime 2}\right\rangle},
$$

where the variance is,

$$
\left\langle C^{\prime 2}\right\rangle=\frac{2}{\delta_{h}} \int_{0}^{\infty} S_{C}\left(k \delta_{h}\right) \mathrm{d}\left(k \delta_{h}\right) .
$$

The wavenumber $k$ is non-dimensionalized by the mean width of the jet as $k \delta_{h}$. 

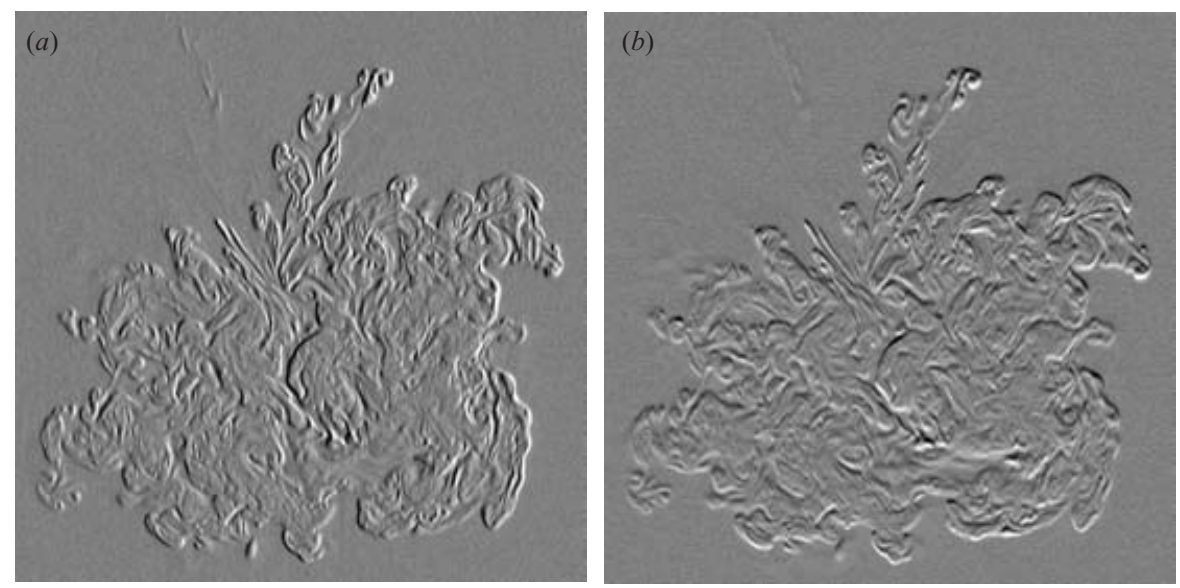

FIGURE 27. Scalar difference field, $\Delta_{r} C$, for increments in different directions at $R e_{j}=10 \times 10^{3}$ and $V_{r}=10$. (a) Horizontal increment of $\boldsymbol{r} / \delta_{h}=0.2 \times 10^{-2} \widehat{z}$. (b) Vertical shift of the same magnitude.

As seen in figure 28, the one-dimensional spectra for vertical and horizontal transects at $R e_{j}=1.0 \times 10^{3}$ are essentially identical at low wavenumbers, but deviate beginning at moderate wavenumbers of $k \delta_{h} \simeq 40$. A deviation between the spectra for horizontal and vertical transects is also seen at moderate wavenumbers for $R e_{j}=10 \times 10^{3}$. The spectra show that less energy is contained in the higher wavenumbers of the vertical transects than the horizontal ones, implying that scalar gradients are larger horizontally than vertically. This is consistent with the observation based on the distributions of scalar increments that the scalar field of the transverse jet has vertically aligned structure at small length scales $(\$ 4.2)$. In the case of the transverse jet, the deviation between vertical and horizontal power spectra begins in the inertial range. The Kolmogorov wavenumber,

$$
k_{\mathrm{K}} \delta_{h} \equiv \frac{\delta_{h}}{\lambda_{\mathrm{K}}} \simeq R e_{\Gamma}^{3 / 4}
$$

is estimated to be $k_{K} \delta_{h} \simeq 118$ for $R e_{j}=1.0 \times 10^{3}$ and $k_{K} \delta_{h} \simeq 665$ for $R e_{j}=10 \times 10^{3}$, while the deviations in the spectra begin begin near $k \delta_{h}=40$ and $k \delta_{h}=16$ for the two Reynolds numbers, respectively. In other experiments, for instance, heated gridturbulence measurements, scalar anisotropy has been seen to begin in the inertial range and continuing into the dissipation range (e.g. Warhaft 2000).

Small-scale anisotropy of the scalar field can also be seen in two-dimensional power spectra of the transverse-jet concentration field. Such power spectra can be calculated from two-dimensional Hann-windowed concentration fields. Twodimensional spectra are computed for each instantaneous image, and ensembleaveraged over 508 realizations at each Reynolds number. The two-dimensional spectra are shown as contour plots of constant scalar power in figure 29. In contrast to the circular contours of the power spectra of an axisymmetric scalar field such as the round jet discharging in a quiescent reservoir (see Catrakis \& Dimotakis 1996), the transverse-jet spectra are anisotropic. In fact, the contours are increasingly elliptical with increasing wavenumbers. The horizontal elongation of the contours once again suggest that scalar gradients are steeper in the horizontal direction than in the vertical 

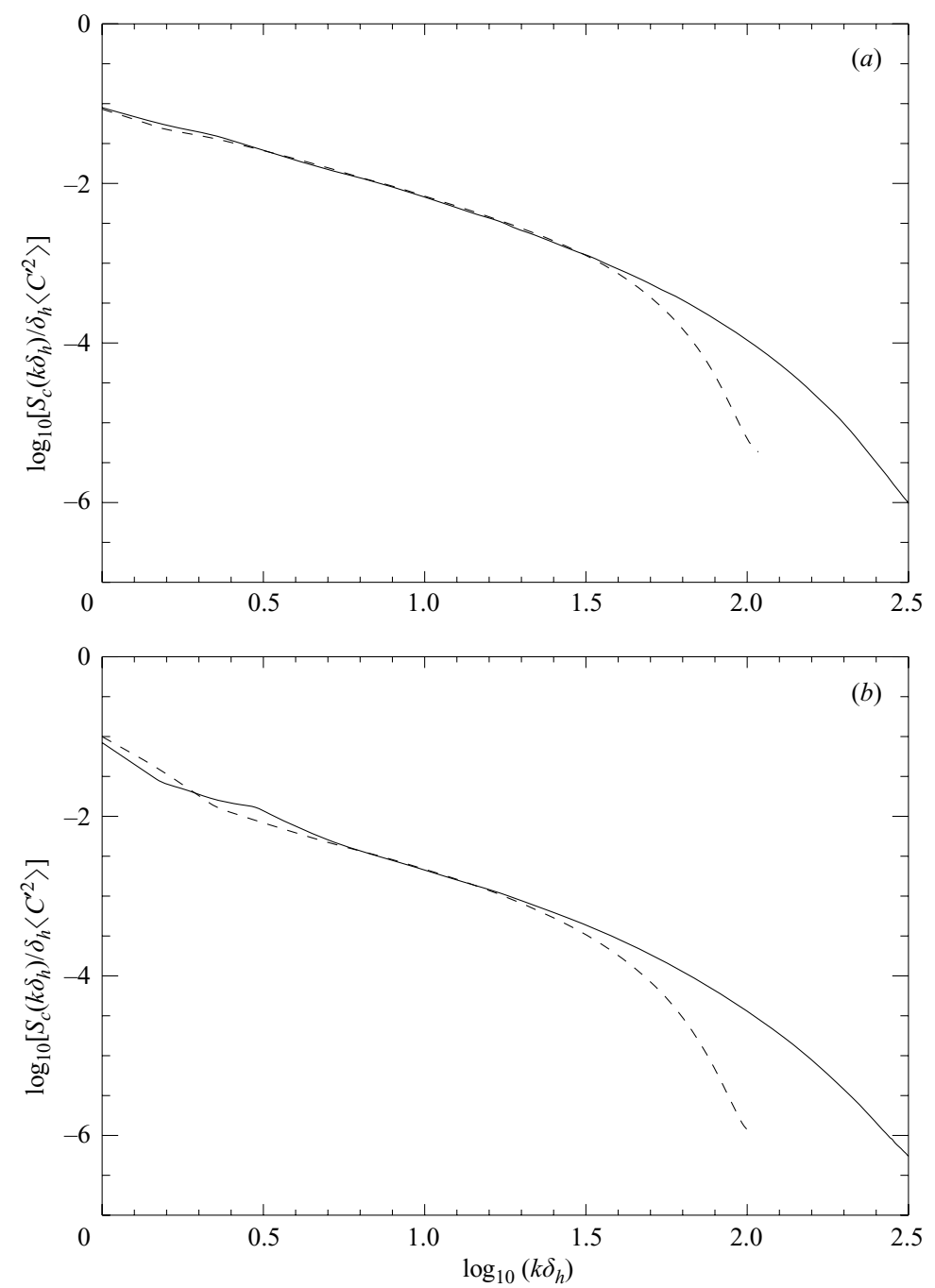

FiguRE 28. Power spectra of one-dimensional transects of the scalar field at $x / d_{j}=50$ for $V_{r}=10$ jet. Solid line is for horizontal transects, and dashed line for vertical transects. Wavenumber, $\boldsymbol{k}$, normalized by the scalar width of the jet, $\delta_{h}$. (a) $R e_{j}=1.0 \times 10^{3}$. (b) $R e_{j}=$ $10 \times 10^{3}$.

direction. For both $R e_{j}=1.0 \times 10^{3}$ and $R e_{j}=10 \times 10^{3}$ jets, the small length scales (higher wavenumbers) differ more, i.e. are more anisotropic, than the large length scales. Further evidence for small-scale anisotropy, and for its spatial localization, are discussed in the following section.

\subsection{Scalar microscale in differing directions}

The scalar microscale, computed for gradients in different directions, is another measure of the over-all anisotropy of the scalar field. It can also be computed for specific regions of the jet to determine whether or not the anisotropy is localized 

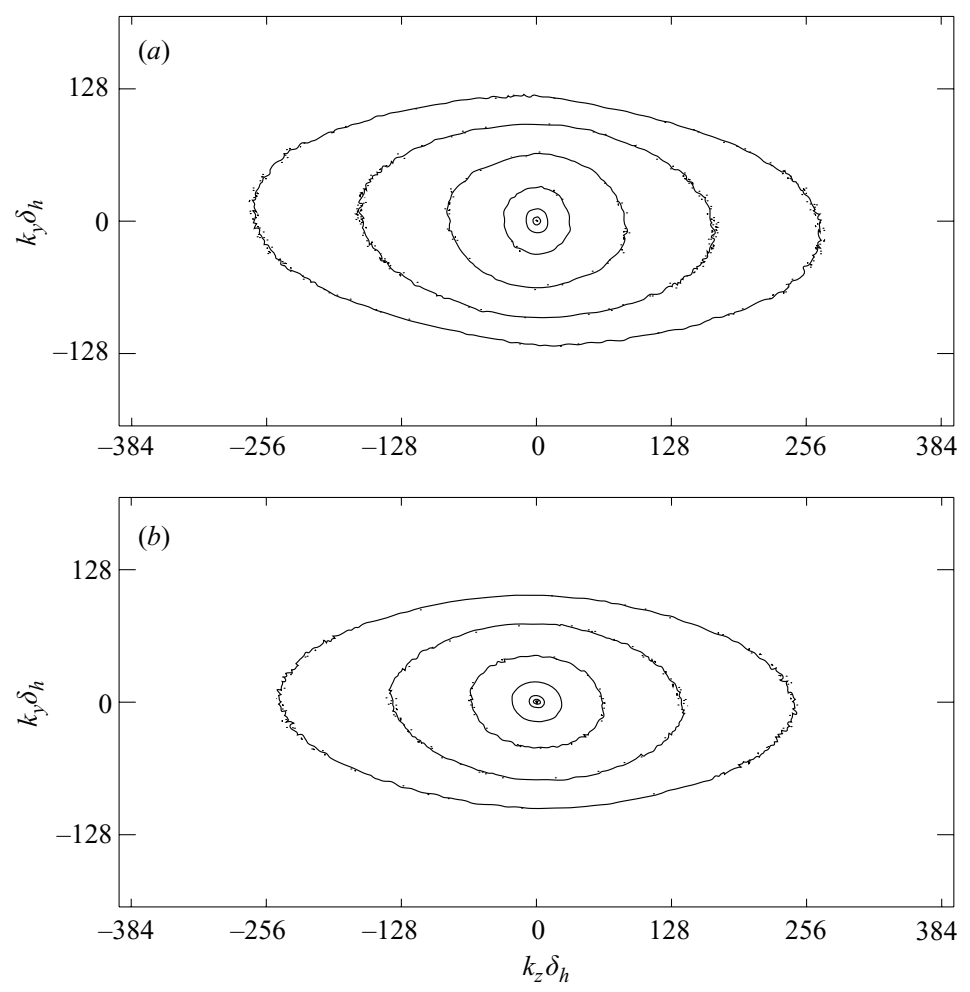

FIGURE 29. Two-dimensional power spectra of the scalar field at $x / d_{j}=50$ for $V_{r}=10$ jet. Wavenumber, $\boldsymbol{k}$, normalized by width of the jet, $\delta_{h}$. Contour plot in $\log _{10}$ increments of 1 . (a) $R e_{j}=1.0 \times 10^{3}$. (b) $R e_{j}=10 \times 10^{3}$.

to specific regions, and help identify possible sources of the observed small-scale anisotropy. A microscale for fluctuations of a scalar field can be defined analogously to the Taylor microscale for velocity fields. For an isotropic scalar field, the scalar microscale, $\lambda_{C}$, is defined as (Tennekes \& Lumley 1972):

$$
\lambda_{C}^{2} \equiv \frac{\left\langle C^{\prime 2}\right\rangle}{\left\langle\left(\partial C^{\prime} / \partial x\right)^{2}\right\rangle} .
$$

For anisotropic scalar fields, a generalized scalar microscale which can vary with direction may be defined as,

$$
\lambda_{C, i}^{2} \equiv \frac{\left\langle C^{\prime 2}\right\rangle}{\left\langle\left(\partial C^{\prime} / \partial x_{i}\right)^{2}\right\rangle},
$$

where $i$ denotes the direction in which the gradient and microscale is computed (Cook \& Dimotakis 2001).

Scalar microscales in two orthogonal directions are computed for measured concentration fields in the transverse jet. The microscales are computed for horizontal and vertical gradients for each concentration field, and then ensemble averaged over 508 images. Figure 30 shows the computed scalar microscales in two directions for $R e_{j}=1,2,5,10$, and $20 \times 10^{3}$. For the Reynolds-number range of the present experiment, the vertical microscales are consistently larger than the horizontal 

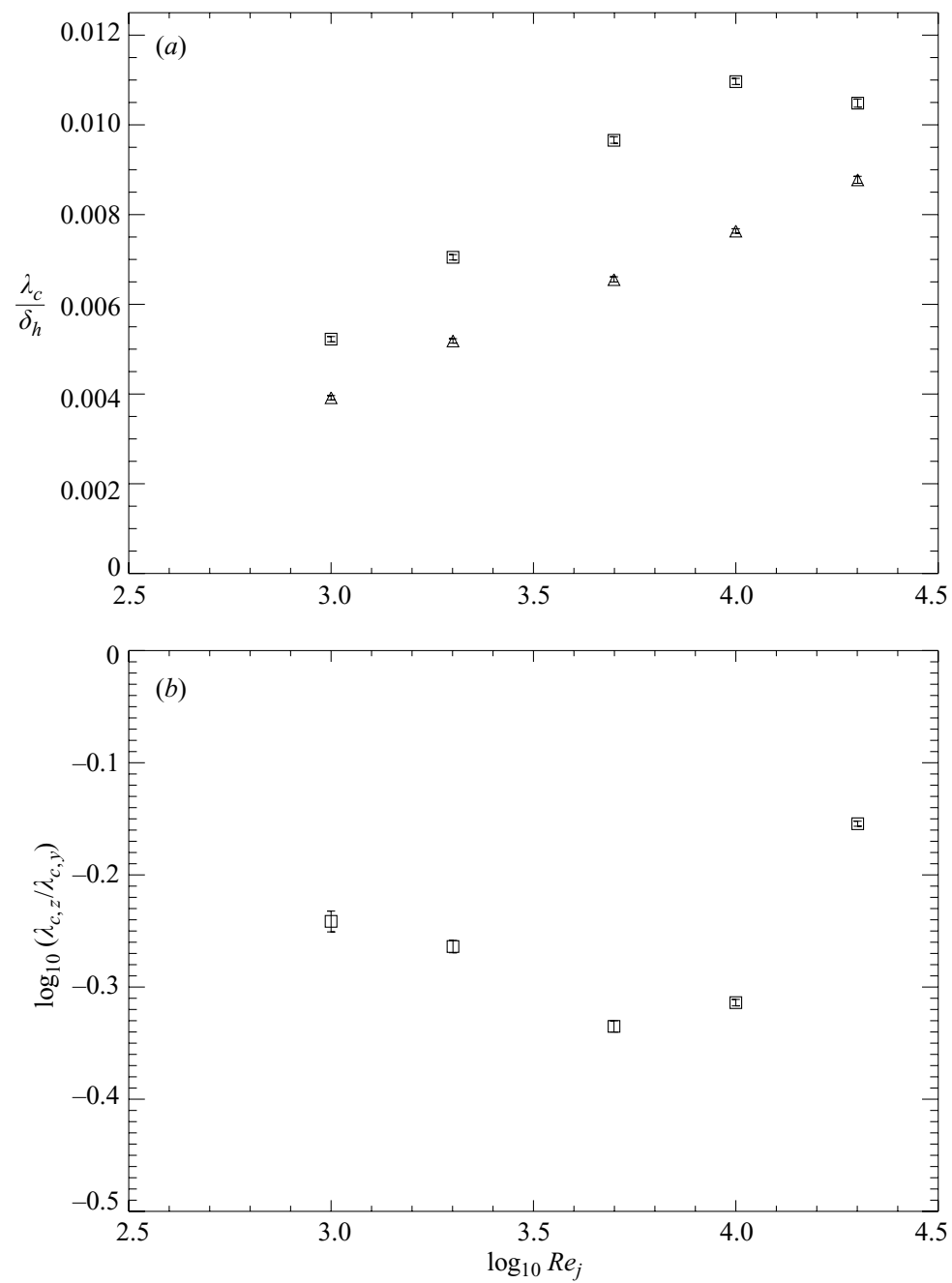

FIGURE 30. Scalar microscale as a function of jet Reynolds number for $V_{r}=10$. (a) Scalar microscale with Reynolds number. $\square$, vertical and $\Delta$, horizontal microscale. (b) Ratio of horizontal to vertical microscales (logarithmic coordinates).

microscales. Ratios of horizontal to vertical microscales are plotted in figure 30 for varying Reynolds number. The degree of anisotropy increases with Reynolds number until $R e_{j} \simeq 10 \times 10^{3}$, and then appears to decrease with further increases in Reynolds number. The evidence is thus that the vertical length scales are larger than the horizontal length scales, which is entirely consistent with the indications of the power spectra and the directional PDFs of scalar increments. Similar anisotropy in the scalar microscales, persisting as the flow developed, was found in direct numerical simulations of three-dimensional Rayleigh-Taylor flow (Cook \& Dimotakis 2001). In the Rayleigh-Taylor flow, the transverse scales (in the direction of gravity) were found to be larger than the horizontal scales. Moreover, the vertical length scales of the scalar field, unlike the horizontal scales, did not collapse with mixing-zone height for different initial conditions in that flow. The Rayleigh-Taylor flow is driven by a directed body force, and the dynamics responsible for generation of vertical 


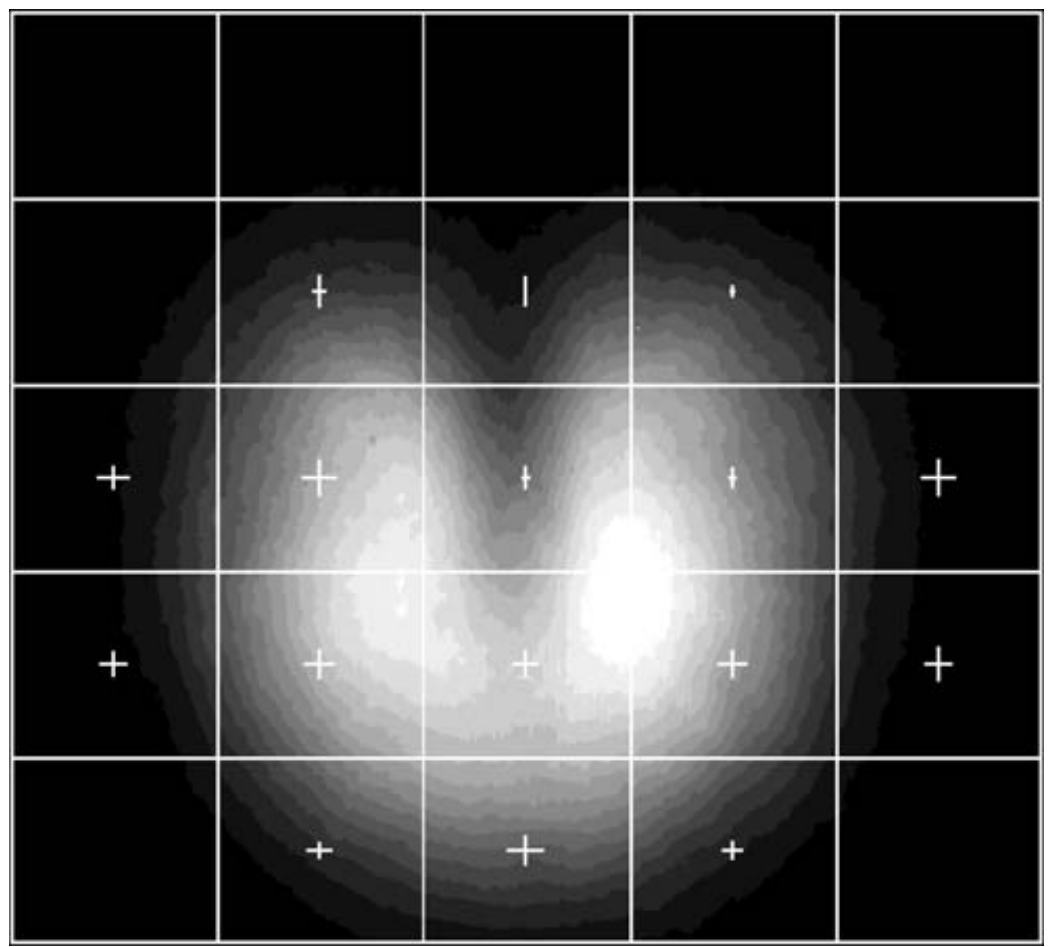

FIGURE 31. Scalar microscales in various regions of the $V_{r}=10$ jet at $R e_{j}=20 \times 10^{3}$, superimposed on the mean concentration field. Microscales are computed in each section and ensemble averaged over 508 images. Crosses have dimensions $4 \lambda_{C, z} \times 4 \lambda_{C, y}$.

scales, even in the interior of the mixing zone, are coupled to the outer scales. In the Rayleigh-Taylor flow the indications are that the small length scales of the scalar field are anisotropic, at least in part, because they are coupled to anisotropic outer scales.

To localize the small-scale anisotropy and perhaps identify a coupling to outer scales which could explain it, the scalar microscale can be computed in specific regions of the jet. The instantaneous scalar field is subdivided into 16 smaller regions, so that the scalar microscales in two orthogonal directions can be computed for each section of each image. The instantaneous local microscales for each region are then ensemble averaged over 508 images. Figure 31 shows the scalar microscales computed for various subsections of the jet, superimposed on an image of the mean concentration field. A cross in each region represents the computed horizontal and vertical microscales within that region. The horizontal and vertical dimensions of each cross are four times the vertical and horizontal scalar microscales computed at each location, i.e. each cross has height and width $4 \lambda_{C, z} \times 4 \lambda_{C, y}$. As seen in figure 31 , differences between the vertical and horizontal microscales are greatest in the uppercentral portion of the jet, in the wake region between the vortex centres. This location is where thin, vertical filaments, or 'fingers', of jet-fluid were seen in the wake of the transverse jet, as discussed in $\S 2$. In the following section, a possible explanation is presented for the observed small-scale anisotropy in the wake region of the transverse jet. 
(a)

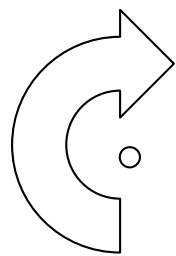

Wall

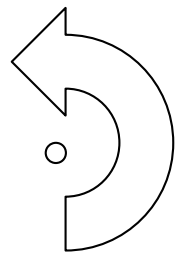

(b)

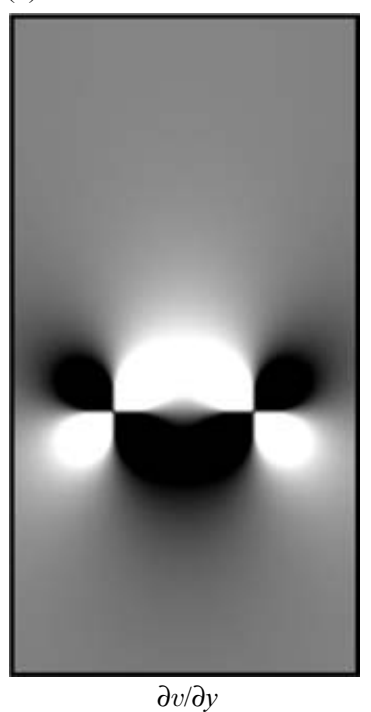

(c)

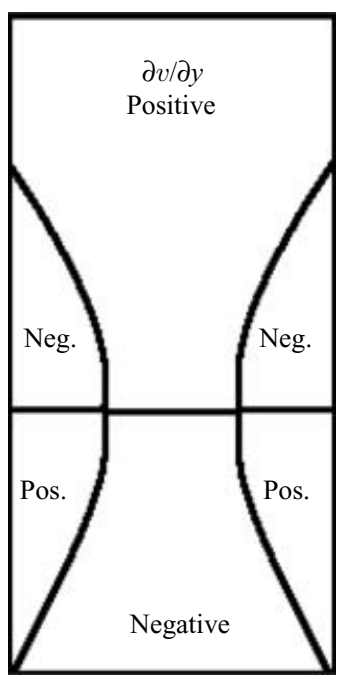

FIGURE 32. The vertical strain rate $\partial v / \partial y$ that would be induced by inviscid flow of counter-rotating line vortices close to a wall. The no-through-flow condition at the wall is satisfied using image vortices. (a) Location and sense of rotation of the line vortices. (b) Vertical strain-rate field $\partial v / \partial y .(c)$ Boundaries of extensional and compressive vertical strain.

\subsection{Origins of anisotropy}

The location of the greatest difference between horizontal and vertical microscales suggests that the observed scalar anisotropy may be connected to the large-scale dynamics of the transverse jet. The dynamics of the far field of the transverse jet have been modelled as a counter-rotating vortex pair (Broadwell \& Breidenthal 1984). Hasselbrink \& Mungal (2001) suggest that a more realistic picture of the vorticity field is an ensemble of stretched vortex rings, inclined at an angle of $\tan ^{-1}\left(1 / V_{r}\right)$ to the crossflow direction. For high-velocity-ratio jets, the angle of inclination is slight $\left(5.7^{\circ}\right.$ for $\left.V_{r}=10\right)$, and Hasselbrink \& Mungal's model of the vorticity field approaches that of a pair of line vortices nearly parallel to the wall.

Thus, as a lowest-order approximation, the large-scale dynamics of the transverse jet are represented as two infinite line vortices close to a wall. Assuming inviscid flow, the vertical strain rate $\partial v / \partial y$ in the far field of the transverse jet can be computed. Figure 32 shows the vertical strain field produced by such an arrangement of vortices. In figure $32(b)$, the strain rate is represented by intensity, with the brightest regions showing the highest extensional strain $(\partial v / \partial y>0)$, and darkest regions showing the greatest compressive $(\partial v / \partial y<0)$ vertical strain. Boundaries between regions of extension and compression are also shown. As seen in the figure, positive strain is generated in the wake region, with peak extensional strain produced close to the jet centreline. The positive strain in the vertical direction is hypothesized to stretch the scalar field, thus producing the thin vertical filaments, or 'fingers' seen in the wake of the jet. Although the line vortex pair is an admittedly simple model for vorticity in the transverse jet, the general behaviour should hold for the real flow. A downward (injection direction) velocity is induced between the regions of opposite-signed vorticity, and this downwash, coupled with a no-through-flow condition imposed by the wall, necessarily produces extensional strain in the vertical 
direction in the wake. The correlation between the observed anisotropy of the scalar field and location of the maximum vertical strain rate suggests that the small-scale anisotropy is a consequence of the large-scale dynamics of the transverse jet.

Although these observations are based on measurements at $x / d_{j}$ of the $V_{r}=10$ transverse jet, the observed local anisotropy of the scalar field is likely to be characteristic of the far field of high-velocity-ratio transverse jets in general, since the counter-rotating vortex pair is a robust feature of the far field of strong transverse jets. In addition, the conclusion that the large-scale dynamics can impose itself on the small scales of the scalar field has possible implications for other turbulent mixing flows. One consequence is that the small-scale structure of turbulent mixing is likely to depend on the flow geometry. This is consistent with the results of mixing experiments by Villermaux, Innocenti \& Duplat (2001) in a turbulent jet, which showed that the injection features of the scalar persist. (The mixing time of the axisymmetric jet was found by Villermaux et al. (2001) to scale with the injection scale, independent of Reynolds number. In addition, the dependence of the mixing times on scalar diffusivity was reported to be a weak (logarithmic) function of Schmidt number.) Another consequence of the apparent connection between large and small scales is that scalar fields produced by turbulent mixing may be anisotropic at small length scales, as observed not only in the transverse jet, but also for other turbulent flows. For instance, as previously discussed, a link has been reported between large-scale anisotropic dynamics and the small scales of the scalar field (even within the mixing zone) for Rayleigh-Taylor flows (Cook \& Dimotakis 2001). The link between large and small scales has also been noted in other flows such as heated grid turbulence (Warhaft 2000). The heated grid-turbulence experiments, of course, were quite different from scalar mixing of the transverse jet. In particular, for the heated grid-turbulence flows, the velocity and vorticity fields were isotropic, but the scalar field had an initial large-scale anisotropy (a linear temperature profile) which manifested itself in smallscale scalar anisotropy. In contrast, the transverse jet has large organized vorticity which produces small-scale anisotropy in the scalar field. In both cases, however, the source for the small-scale anisotropy is postulated to be a coupling between large and small scales of the turbulent mixing flows.

\section{Conclusions}

In summary, enhanced mixing, in the sense of better spatial homogenization of the scalar field, is found with increasing Reynolds number in the far field of highvelocity ratio liquid-phase transverse jets over the range $1.0 \times 10^{3} \leqslant R e_{j} \leqslant 20 \times 10^{3}$. Many classical measures, such as scalar trajectories, decay of mean concentration on the centreline, and the spatial extent of the jet, are essentially independent of Reynolds number. However, the mixed-fluid distribution, as quantified by the PDF of jet-fluid concentration, evolves with Reynolds number at fixed location in the far field of the transverse jet. With the enhanced stirring that comes with increasing Reynolds number, the scalar field develops a preferred concentration, and the jet-fluidconcentration PDF evolves from a monotonically decreasing function to a stronglypeaked distribution. This behaviour, which occurs despite the greater entrainment rate of the transverse jet (at $x / d_{j}=50$ ) as compared to the jet discharging in a quiescent reservoir, suggests that the transverse jet is an efficient mixer.

Whole-field scalar increments show the concentration field of the turbulent transverse jet to be intermittent, with plateaux of nearly constant concentration separated by cliffs of sharp changes in concentration. The distribution of scalar 
differences is seen to tend toward long-tailed exponential distributions with decreasing separation distance. These long-tailed PDFs of scalar differences, which persist for the Reynolds number range studied, are similar to results reported for grid-turbulence, Rayleigh-Bénard convection, and other turbulent flows. The long exponential tails and strongly peaked shape of the PDF of scalar differences is consistent with the physical picture of well-mixed plateaux, separated by cliffs, in the scalar field.

The scalar field of the liquid-phase high-Schmidt-number transverse jet is found to be anisotropic at even the smallest length scales. For instance, scalar power spectra for the far field of the jet are found to be essentially axisymmetric for lower wavenumbers, but increasingly elliptical for higher wavenumbers. The smallscale anisotropy is also seen in the difference between PDFs of scalar increments in different directions. Probability distributions of scalar increments have narrower shapes for vertical separations than for horizontal separations. These results indicate that the scalar field has less variation (in terms of PDFs of scalar increments, and power spectra) in the vertical direction than in the horizontal direction. Thus, the fine scales of the concentration field appear to have preferential orientation in the vertical direction. The small-scale anisotropy of the scalar field persists from the lowest Reynolds numbers investigated, $R e_{j}=1.0 \times 10^{3}$, to the highest Reynolds numbers studied, $R e_{j}=20 \times 10^{3}$. Further evidence for the anisotropy of the scalar field is seen in the scalar microscales computed for different directions. The difference between scalar microscales in two directions is greatest in the wake region of the jet, between the body of the jet and the wall. The observed local anisotropy is believed to be a consequence of the strain field produced by the counter-rotating vorticity in the transverse jet. Thus, the large-scale vortex structure of the transverse jet appears to impose itself on even the smallest features of the advected scalar field. The scalar anisotropy that is found in the $V_{r}=10$ jet is expected in other high-velocity-ratio jets, since the counter-rotating vortex pair is a characteristic feature of strong transverse jets.

One consequence of the apparent link between large and small scales that is observed in these experiments (and which has been previously reported for other turbulent flows) is that local scalar isotropy need not exist for turbulent mixing flows having anisotropic large scales. In addition, the long-tailed and peaked scalarincrement distribution found in the transverse jet is a consequence of intermittent, well-mixed plateaux in the scalar field separated by cliffs, and thus can be found in many other turbulent mixing flows. Furthermore, as a consequence of the connection between large-scale dynamics and the small scales of the scalar field, the detailed structure of turbulent mixing may depend on flow geometry. These conclusions, which are drawn from the study of liquid-phase transverse jets up to Reynolds numbers of $20 \times 10^{3}$, add to the existing evidence that the universality of small scales of the scalar field is not typical of turbulent-mixing flows. Additional experimental and numerical work is desirable to further analyse the behaviour of the transverse jets and other turbulent-mixing flows at yet higher Reynolds numbers and different Schmidt or Prandtl numbers.

We are grateful for assistance of D. Lang with the Cassini digital imaging system, and of S. Lombeyda with the three-dimensional visualizations. We also thank P. Svitek for his help with mechanical design and the operation of the GALCIT freesurface water tunnel. M. Gharib, H. Hornung, A. Leonard and Z. Warhaft provided helpful comments on the text. This work was supported by the Air Force Office of Scientific Research (F49620-98-1-0052 and F49620-01-1-0006) and a NDSEG 
fellowship. J.W.S. acknowledges the support of P. Atsavapranee and I.-Y. Koh for the revision of this text. Development of the Cassini system was supported by DURIP grant F49620-95-1-0199, and AFOSR grants F49620-94-1-0283 and F49620-00-10036. The three-dimensional visualizations were mode possible by NSF MRI grant 0079871.

\section{Appendix. PDFs for higher-dimensional diffusive fields}

The PDF for a distribution, $C(\boldsymbol{r})$, in $d$-dimensional space is the differential $d$-dimensional volume, $\mathrm{d} V_{d}(C)$, associated with a differential concentration, $\mathrm{d} C$, equation (16b).

Normalization by the total volume, $V_{d, t o t}$, is necessary so that the PDF has the properties discussed in $\S 3$, and proper dimensions of inverse concentration.

Consider, for instance, the Gaussian scalar distribution,

$$
C(r, t)=\frac{1}{2^{d}(\pi \mathscr{D} t)^{d / 2}} \exp \left(-r^{2} / 4 \mathscr{D} T\right)=\frac{1}{(2 \pi)^{d / 2} \sigma^{d}} \exp \left(-r^{2} / 2 \sigma^{2}\right) .
$$

This is the solution to the $d$-dimensional initial-value problem for the diffusion equation on an infinite domain with initial conditions of a delta function at the origin, $C(\boldsymbol{r}, t=0)=\delta(\boldsymbol{r})$. Here, $\sigma^{2}=2 \mathscr{D} t$. The differential length, $\mathrm{d} r$, at any given time (i.e. $\sigma=$ constant) is related to $\mathrm{d} C$ by

$$
\frac{\mathrm{d} r}{\mathrm{~d} C}=-\frac{(2 \pi)^{d / 2} \sigma^{d}}{2 r} \exp \left(r^{2} / 2 \sigma^{2}\right) .
$$

Using (A 1), $\mathrm{d} r / \mathrm{d} C$ can be written as a function of $C$ alone:

$$
\frac{\mathrm{d} r}{\mathrm{~d} C}=\frac{1}{2 C r}=\frac{1}{2 \sigma C\left\{-2 \ln \left[(2 \pi)^{d / 2} \sigma^{d} C\right]\right\}^{1 / 2}} .
$$

The differential $d$-dimensional volume $\mathrm{d} V_{d}(C)$ may then be expressed as a function of $C$ using (A 1) and (A2) to yield the form of the PDF for a $d$-dimensional Gaussian distribution:

$$
f_{d}(C) \propto \frac{[\ln (1 / C)]^{d / 2-1}}{C} .
$$

For one-, two- and three-dimensional Gaussian concentration distributions, the PDFs are then:

$$
\begin{gathered}
f_{1}(C) \propto \frac{1}{C[\ln (1 / C)]^{1 / 2}} . \\
f_{2}(C) \propto \frac{1}{C} . \\
f_{3}(C) \propto \frac{[\ln (1 / C)]^{1 / 2}}{C} .
\end{gathered}
$$

These functions are shown in figure 33. The distribution for the one-dimensional case has two peaks, at $C=0$ and $C=1$, while the distributions for the two- and three-dimensional cases each have a peak at $C=1$. The PDF decreases monotonically to zero at $C=1$ for the three-dimensional Gaussian, and to a non-zero minimum for the two-dimensional Gaussian.

Since $d$-dimensional transects of a higher-dimensional Gaussian are themselves $d$-dimensional Gaussians, the PDFs above can be viewed as PDFs of concentration 


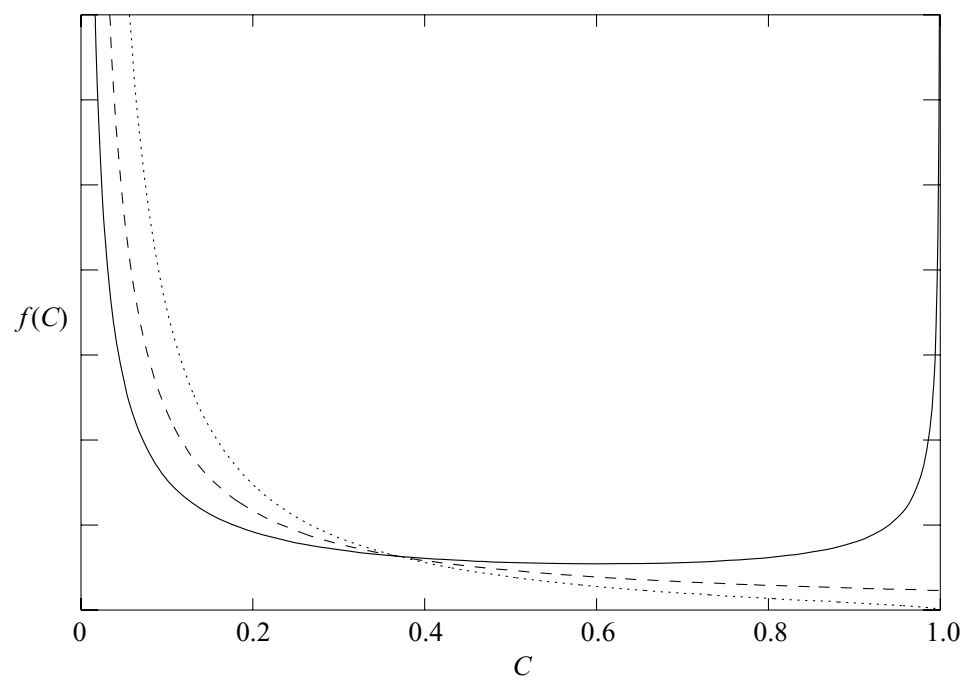

FIGURE 33. PDFs of diffusive (Gaussian) distributions. Solid line: one-dimensional; dashed line: two-dimensional; dotted line: three-dimensional.

distributions in $d$-dimensional space, or as $d$-dimensional measurements of a higherdimensional distribution. Thus, a PDF can depend on the dimensionality of the space (or measurement), as well as the concentration field, $C(\boldsymbol{r})$.

\section{REFERENCES}

Andreopoulos, J. \& Rodi, W. 1984 An experimental investigation of jets in a crossflow. J. Fluid Mech. 138, 93-127.

Antonia, R. A., Browne, L. W. B., Britz, D. \& Chambers, A. J. 1984 A comparison of properties of temporal and spatial temperature increments in a turbulent plane jet. Phys. Fluids 27, 87-93.

Antonia, R. A., Chambers, A. J., Britz, D. \& Browne, L. W. B. 1986 Organized structures in a turbulent plane jet: topology and contribution to momentum and heat transport. J. Fluid Mech. 172, 211-229.

BREIDENTHAL, R. E. 1981 Structure in turbulent mixing layers and wakes using a chemical reaction. J. Fluid Mech. 109, 1-24.

Broadwell, J. E. \& Breidenthal, R. E. 1984 Structure and mixing of a transverse jet in incompressible flow. J. Fluid Mech. 148, 405-412.

Budwig, R., Tavoularis, S. \& Corrsin, S. 1985 Temperature fluctuations and heat flux in gridgenerated isotropic turbulence with streamwise and transverse mean-temperature gradients. J. Fluid Mech. 153, 441-460.

Catrakis, H. J. \& Dimotakis, P. E. 1996 Mixing in turbulent jets: scalar measures and isosurface geometry. J. Fluid Mech. 317, 369-406.

Chassaing, P., George, J., Claria, A. \& Sananes, F. 1974 Physical characteristics of subsonic jets in a cross-stream. J. Fluid Mech. 62, 41-64.

Ching, E. S. C. 1991 Probabilities for temperature differences in Rayleigh-Bénard convection. Phys. Rev. A 44, 3622-3629.

Cook, A. W. \& Dimotakis, P. E. 2001 Transition stages of Rayleigh-Taylor instability between miscible fluids. J. Fluid Mech. 443, 69-99.

Corrsin, S. 1951 On the spectrum of isotropic temperature fluctuations in isotropic turbulence. J. Appl. Phys. 22, 469-473.

Cortelezzi, L. \& Karagozian, A. R. 2001 On the formation of the counterrotating vortex pair in transverse jets. J. Fluid Mech. 446, 347-373. 
Dahm, W. J. A. \& Dimotakis, P. E. 1990 Large Schmidt number mixing of a conserved scalar in the self-similar far field of turbulent jets. J. Fluid Mech. 217, 299-330.

Dimotakis, P. E. 1986 Two-dimensional shear-layer entrainment. AIAA J. 24, 1791-1796.

Dimotakis, P. E. 2000 The mixing transition in turbulence. J. Fluid Mech. 409, 69-98.

Dimotakis, P. E. \& CATRakis, H. J. 1996 Turbulence, fractals, and mixing. NATO Advanced Studies Institute series, Mixing: Chaos and Turbulence, GALCIT Rep. FM97-1.

Dimotakis, P. E. \& Hall, J. L. 1987 A simple model for finite chemical kinetics analysis of supersonic turbulent shear layer combustion. AIAA/SAE/ASME/ASEE 23rd Joint Propulsion Meeting, Paper 87-1879.

Dimotakis, P. E. \& Miller, P. L. 1990 Some consequences of the boundedness of scalar fluctuations. Phys. Fluids A 2, 1919-1920.

ECKART, C. 1948 An analysis of the stirring and mixing processes in incompressible fluids. J. Mar. Res. 7, 265-275.

FeARn, R. \& Weston, R. P. 1974 Vorticity associated with a jet in a cross flow. AIAA J. 12, $1666-1671$.

Fric, T. F. \& Roshko, A. 1994 Vortical structure in the wake of a transverse jet. J. Fluid Mech. 279, $1-47$.

Friehe, C. A., Van Atta, C. W. \& Gibson, C. H. 1971 Jet turbulence: Dissipation rate measurements and correlations. Turbulent Shear Flows AGARD Conf. Proc. 93, 18.1-7.

Frisch, U. 1995 Turbulence. Cambridge University Press.

Gibson, C. H., Friehe, C. A. \& McConnell, S. O. 1977 Structure of sheared turbulent fields. Phys. Fluids 20, part II, S156-S167.

Gordier, R. L. 1959 Studies on fluid jets discharging normally into moving liquid. St Anthony Falls Hyd. Lab., U. Minnesota, Tech. Paper 28, Series B.

Gruber, M. R., Baurle, R. A., Mathur, T. \& Hsu, K.-Y. 1999 Fundamental studies of cavitybased flameholder concepts for supersonic combustors. AIAA/SAE/ASME/ASEE 23rd Joint Propulsion Meeting, Paper 99-2248.

Han, D., Orozco, V. \& Mungal, M. G. 2000 Gross-entrainment behavior of turbulent jets injected obliquely into a uniform crossflow. AIAA J. 38, 1643-1649.

Hasselbrink, E. F. \& Mungal, M. G. 2001 Transverse jets and jet flames. Part 1. Scaling laws for strong transverse jets. J. Fluid Mech. 443, 1-25.

Holzer, M. \& Siggia, E. D. 1994 Turbulent mixing of a passive scalar. Phys. Fluids 6, 1829-2176.

Johari, H., Pacheco-Tougas, M. \& Hermanson, J. 1999 Penetration and mixing of fully modulated turbulent jets in crossflow. AIAA J. 37, 842-850.

Kamotani, Y. \& Greber, I. 1972 Experiments on a turbulent jet in a cross flow. AIAA J. 10, $1425-1429$.

Karagozian, A. R. 1986 An analytical model for the vorticity associated with a transverse jet. AIAA J. 24, 429-436.

Keffer, J. F. \& Baines, W. D. 1963 The round turbulent jet in a cross-wind. J. Fluid Mech. 15, 481-496.

Kelso, R. M., Lim, T. T. \& Perry, A. E. 1996 An experimental study of round jets in cross-flow. J. Fluid Mech. 306, 111-144.

Kelso, R. M. \& Smits, A. J. 1995 Horseshoe vortex systems resulting from the interaction between a laminar boundary layer and a transverse jet. Phys. Fluids 7, 153-158.

Koochesfahani, M. M. \& Dimotakis, P. E. 1986 Mixing and chemical reactions in a turbulent liquid mixing layer. J. Fluid Mech. 170, 83-112.

Kuznetsov, V. R. \& Sabel’nikov, V. A. 1990 Turbulence and Combustion. Hemisphere.

Kuzo, D. M. 1995 An experimental study of the turbulent transverse jet. PhD thesis, California Institute of Technology, Pasadena, CA.

Lozano, A., Smith, S. H., Mungal, M. G. \& Hanson, R. K. 1993 Concentration measurements in a transverse jet by planar laser-induced fluorescence of acetone. AIAA J. 32, 218-221.

McMahon, H. M., Hester, D. D. \& Palfery, J. G. 1971 Vortex shedding from a turbulent jet in a cross-wind. J. Fluid Mech. 48, 73-80.

Margason, R. J. 1993 Fifty years of jet in cross flow research. AGARD Meeting on Computational and Experimental Assessment of Jets in Cross Flow, April, 1993.

Mestayer, P. G., Gibson, C. H., Coantic, M. F. \& Patel, A. S. 1976 Local anisotropy in heated and cooled turbulent boundary layers. Phys. Fluids 19, 1279-1287. 
Miller, P. L. \& Dimotakis, P. E. 1991 Reynolds number dependence of scalar fluctuations in a high Schmidt number turbulent jet. Phys. Fluids A 3, 1156-1163.

Morton, B. R. \& Ibbetson, A. 1996 Jets deflected in a crossflow. Exp. Thermal Fluid Sci. 12, $112-133$.

Moussa, Z. M., Trischka, J. W. \& EskinaZi, S. 1977 The near field in the mixing of a round jet with a cross-stream. J. Fluid Mech. 80, 49-80.

Mungal, M. G. \& Lozano, A. 1996 Some observations of a large, burning jet in crossflow. Exps. Fluids 21, 264-267.

MydLARSKI, L. \& WARHAFT, Z. 1998 Three-point statistics and the anisotropy of a turbulent passive scalar. Phys. Fluids 10, 2885-2894.

Niederhaus, C. E., Champagne, F. H. \& Jacobs, J. W. 1997 Scalar transport in a swirling transverse jet. AIAA J. 35, 1697-1704.

Oвоuкhov, A. M. 1949 Structure of the temperature field in turbulent flows. Izv. Akad. Nauk SSSR Geogr. Geophys. 13, 58-69.

Ould-Rouis, M., Anselmet, F., Le Gal, P. \& Vaienti, S. 1995 Statistics of temperature increments in fully developed turbulence. Part II. Experiments. Physica D 85, 405-424.

Patrick, M. A. 1967 Experimental investigation of the mixing and penetration of a round turbulent jet injecting perpendicular into a transverse stream. J. Hydronaut. Div. ASCE 45, 16-31.

Pratte, B. D. \& Baines, M. 1967 Profiles of the round turbulent jet in a crossflow. J. Hydronaut. Div. ASCE 92, 53-64.

Pringsheim, P. 1949 Fluorescence and Phosphorescence. Interscience.

Pumir, A. 1994 A numerical study of the mixing of a passive scalar in three dimensions in the presence of a mean gradient. Phys. Fluids 6, 2118-2132.

ReILlY, R. S. 1968 Investigation of the deformation and penetration of a turbulent subsonic jet issuing transversely into a uniform, subsonic main stream. $\mathrm{PhD}$ thesis, University of Maryland.

Ricou, F. P. \& Spalding, D. B. 1961 Measurements of entrainment by axisymmetrical turbulent jets. J. Fluid Mech. 11, 21-32.

Shan, J. W., LANG, D. B. \& Dimotakis, P. E. 2004 Scalar concentration measurements in liquid-phase flows with pulsed lasers. Exps. Fluids, 36, 268-273.

Shraiman, B. \& Siggia, E. 2000 Scalar turbulence. Nature 405, 639-646.

Smith, S. H. \& Mungal, M. G. 1998 Mixing, structure and scaling of the jet in crossflow. J. Fluid Mech. 357, 83-122.

Sreenivasan, K. R. 1991 On local isotropy of passive scalars in turbulent shear flows. Proc. R. Soc. Lond. A 434, 165-182.

Sreenivasan, K. R. \& Antonia, R. A. 1997 The phenomenology of small-scale turbulence. Annu. Rev. Fluid Mech. 29, 435-472.

Sreenivasan, K. R., Antonia, R. A. \& Britz, D. 1979 Local isotropy and large structures in a heated turbulent jet. J. Fluid Mech. 94, 745-775.

Su, L. \& Mungal, M. G. 2004 Simultaneous measurements of scalar and velocity field evolution in turbulent crossflowing jets. J. Fluid Mech. 513, 1-45.

Su, L. K., Han, O., Miraflor, M., \& Mungal, M. G. 2000 Measurements of scalar and velocity fields in turbulent crossflowing jets with low velocity ratio. AIAA Paper 2000-0815

TAVOUlaris, S. \& CoRrsin, S. 1981 Experiments in nearly homogeneous turbulent shear flow with a uniform mean temperature gradient. Part 2. The fine structure. J. Fluid Mech. 104, 349367.

Tennekes, H. \& Lumley, J. L. 1972 A First Course in Turbulence. MIT Press.

Thoroddsen, S. T. \& Van AtTa, C. W. 1992 Exponential tails and skewness of density-gradient probability density functions in stably stratified turbulence. 244, 547-566.

Tong, C. \& Warhaft, Z. 1994 On passive scalar derivative statistics in grid turbulence. Phys. Fluids 6, 2165-2176.

Uberoi, M. S. \& Singh, P. I. 1975 Turbulent mixing in a two-dimensional jet. Phys. Fluids 18, 764-769.

Vanlerberghe, W., Santiago, J., Dutton, J. \& Lucht, R. 2000 Mixing of a sonic transverse jet injected into a supersonic flow. AIAA J. 38, 470-479.

Villermaux, E., Innocenti, C. \& Duplat, J. 2001 Short circuits in the Corrsin-Obukhov cascade. Phys. Fluids 13, 284-289. 
WALKER, D. A. 1987 A fluorescence technique for measurement of concentration in mixing liquids. J. Phys. E: Sci. Instrum. 20, 217-224.

Warhaft, Z. 2000 Passive scalars in turbulent flows. Annu. Rev. Fluid Mech. 32, 203-240.

Xu, X.-H. \& Yeung, E. S. 1997 Direct measurement of single-molecule diffusion and photodecomposition in free solution. Science 275, 1106-1109.

Yoda, M., Hesselink, L. \& Mungal, M. G. 1992 The evolution and nature of large-scale structures in the turbulent jet. Phys. Fluids 4, 803-811.

Yuan, L. L., \& Street, R. L. 1998 Trajectory and entrainment of a round jet in crossflow. Phys. Fluids 10, 23231-2335.

Yuan, L. L., \& Street, R. L. \& Ferziger, J. H. 1999 Large-eddy simulations of a round jet in crossflow. J. Fluid Mech. 379, 71-104. 